\title{
Effects of Flexibility on the Aerodynamic Performance of Flapping Wings
}

\author{
Chang-kwon Kang ${ }^{1}$, Hikaru Aono ${ }^{2}$, Carlos E. S. Cesnik ${ }^{3}$ \\ Department of Aerospace Engineering, University of Michigan, Ann Arbor, MI, 48109 \\ and \\ Wei Shyy ${ }^{4}$ \\ Department of Aerospace Engineering, University of Michigan, Ann Arbor, MI, 48109 \\ Department of Mechanical Engineering, Hong Kong University of Science and Technology, Kowloon, Hong Kong
}

Effects of chordwise, spanwise, and isotropic flexibility on the force generation and propulsive efficiency of flapping wings are elucidated. For a moving body immersed in viscous fluid, different types of forces, as a function of the Reynolds number, reduced frequency $(k)$, and Strouhal number $(S t)$, acting on the moving body are identified based on a scaling argument. In particular, at the Reynolds number regime of $O\left(10^{3}-10^{4}\right)$ and the reduced frequency of $O(1)$, the added mass force, related to the acceleration of the wing, is important. Based on the order of magnitude and energy balance arguments, a relationship between the propulsive force and the maximum relative wing tip deformation parameter $(\gamma)$ is established. The parameter depends on the density ratio, $S t, k$, natural and flapping frequency ratio, and flapping amplitude. The lift generation, and the propulsive efficiency can be deduced by the same scaling procedures. It seems that the maximum propulsive force is obtained when flapping near the resonance, whereas the optimal propulsive efficiency is reached when flapping at about half of the natural frequency; both are supported by the reported studies. The established scaling relationships can offer direct guidance for MAV design and performance analysis.

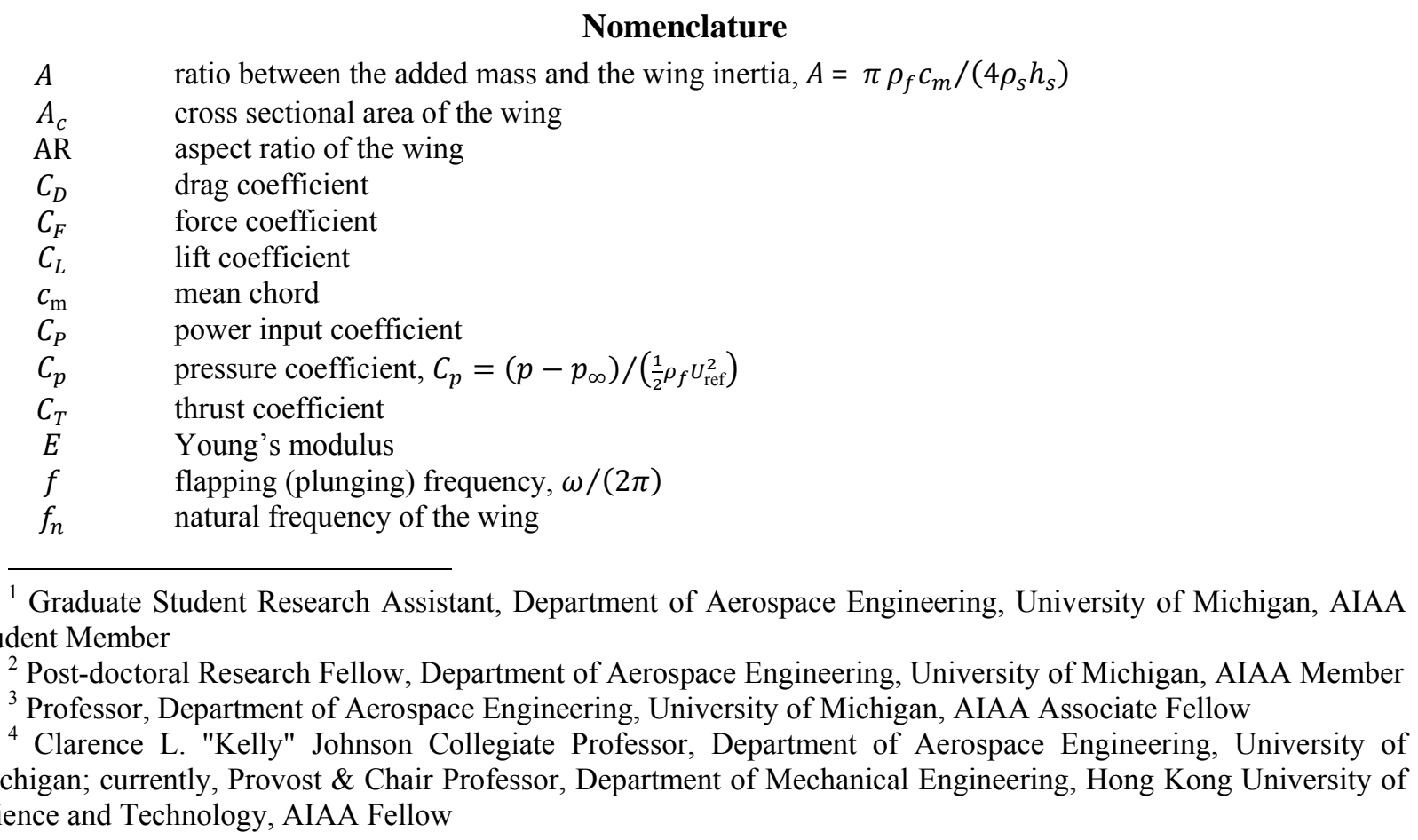




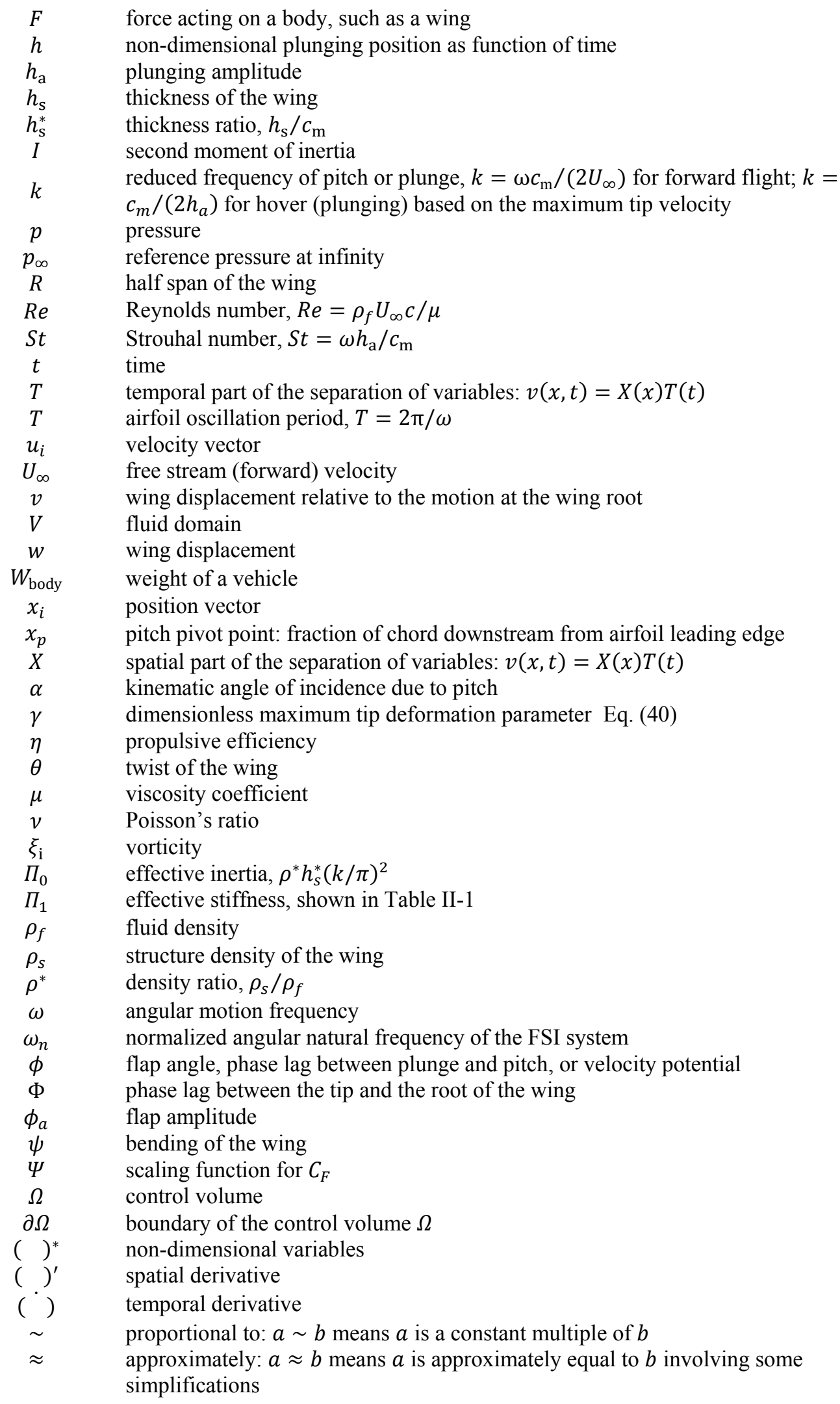




\section{Introduction}

$\mathrm{T}$ HE flapping mechanisms inherent to the biological flyers, such as insects and birds, have inspired the most exotic dreams, ever since the history of human beings, from Daedalus and Icarus in the Greek mythology, via Leonardo Da Vinci's ornithopter, to a recent successfully sustained human powered flapping flight [1] at human scales of $O(1 \mathrm{~m})$. At smaller scales of $15 \mathrm{~cm}$ or less, micro air vehicles (MAVs) are of great interest in remote sensing and information gathering capabilities both in military as well in civilian applications. Smaller sizes and lower flight speeds lead to lower Reynolds numbers and higher sensitivity to wind gust effects than for the conventional airplanes. Furthermore, since MAVs are of light weight and fly at low speeds, they are sensitive to wind gust, and their wing structures are often flexible and tend to deform significantly during flight $[2,3,4,5,6]$. Because of the common characteristics shared by MAVs and biological flyers, the aerospace and biological science communities are now actively communicating and collaborating.

Recently, a bio-inspired bird-like MAV has successfully demonstrated its ability to hover (Nano Hummingbird) [7]. At larger scales of wing span of two meters (SmartBird) [8] a bird-like flapping motion was integrated into fully automated robotic flyer. Other examples of MAVs are the Delfly [9], Microrobotic Fly [10], among others. In order to operate under wind gust, to avoid objects, or to hover, highly deformed wing shapes and coordinated wing-tail movement in the biological flight are often observed. Understanding the aerodynamic, structural, and control implications of these modes is essential for the development of high performance and robust flapping wing MAVs for accomplishing desirable missions. Moreover, the large flexibility of the wings leads to complex fluid-structure interactions, while the kinematics of flapping and the spectacular maneuvers performed by natural flyers result in highly coupled nonlinearities in fluid dynamics, structural dynamics, flight dynamics, and control systems.

As reviewed and illustrated by numerous studies $[3,6,11,12,13,14,15,16,17,18]$, biological flyers showcase desirable flight characteristics and performance objectives and the strategies exhibited in nature have the potential to be utilized in the design of flapping wing MAVs. In particular, wing flexibility is likely to have a significant influence on the resulting aerodynamics. Based on a literature study, it was found that several questions in flexible wing aerodynamics have not been adequately addressed in the existing literature, among which, the key ones include: (i) What are the dominant non-dimensional parameters, such as the Reynolds number $(R e)$ that indicates the ratio of the fluid dynamic inertial forces to the viscous forces, the Strouhal number $(S t)$ giving the ratio between the wing velocity to the fluid convection velocity, the reduced frequency $(k)$ that measures the unsteadiness of the flow, and the effective stiffness defined as the wing rigidity normalized by the fluid dynamic pressure, etc., for the aerodynamic performance of flexible flapping wings? (ii) What are the underlying mechanisms of force generation or propulsive efficiency of flexible flapping wings? (iii) Do optima exist for the aerodynamic performances, and if so, what are these? This study explores these questions using high-fidelity computational models and analytic scaling methods.

Scaling parameters resulting from dimensional analysis help identify key characteristics of the model, via Buckingham's $\Pi$-theorem, which also reduces the number of involved parameters to the sufficient number of combinations $[19,20,21,22]$. Under certain circumstances, the result obtained from the dimensional analysis, can be reduced to a simpler relationship, with a reduced number of arguments, as a property of the special problem under consideration. The non-dimensional parameters arising from such a scaling analysis can identify similarity variables, which can be of critical value even if a complete mathematical solution is missing [19].

In the field of flexible flapping wing aerodynamics numerous efforts using scaling arguments have increased our knowledge of the complex interplay between flexibility and resulting aerodynamics. Depending on the type of the model and the governing equations the resulting set of scaling parameters may vary. For example, for flexible flapping wings, Shyy et al. [6] considered the Navier-Stokes equation for out-of-plane motion of an isotropic flat plate, and Ishihara et al. [23] investigated the Navier-Stokes equation along with the linear isotropic elasticity equations to study the effects of flexibility on wing pitch changes in dipteran flapping flight. Furthermore, Ishihara et al. [24] have measured the lift generated by a dynamically scaled flexible wing model. They introduced the Cauchy number that describes the ratio between the fluid dynamic pressure and elastic reaction force and presented correlation between time-averaged lift and the Cauchy number. More recently, Thiria and Godoy-Diana [25] and Ramananarivo, Godoy-Diana, and Thiria [26] have measured the thrust and the propulsive efficiency of a selfpropelled flapping flyer with flexible wings in air. Since the density ratio is high, the elastic deformation of the wing was mostly balanced by the wing inertia. They have introduced the elastoinertial number using scaling arguments to define the ratio between the inertial forces and the elastic restoring forces and showed that the measured thrust scales with the elastoinertial number. Furthermore, in Ref. [26] the cubic nonlinear damping term due to the aerodynamics was linked to the effects of flexibility on the aerodynamic performance. It should be mentioned that these two studies only consider a portion of the parameter-space and for example the effects of density ratios on the 
force generation of flexible flapping wings have not been considered. Hence, the parameter-space involving the scaling parameters for the fluid-structure interaction needs to be mapped out in a systematic fashion to understand the role of flexibility and density ratio on the fluid dynamic force generations and the propulsive efficiency of coupled systems.

Recent review by Shyy et al. [6] and experimental study by Ramananarivo, Godoy-Diana, and Thiria [26] have offered surveys of the current state of the investigation of the role of the flexibility on the fluid dynamic performance of flapping wings. The maximum propulsive force, such as thrust in forward flight or lift in hover motion, was generated at a frequency which was slightly lower than the natural frequency of the system $[27,28,25,26,29,30]$. Zhang, Liu, and Lu [27] studied numerically using the lattice Boltzmann method a flexible flat plate modeled as a rigid plate with a torsional spring at the pivot point on the leading edge of the wing. They conclude that the flat plate would move forward, hence generate thrust when the leading edge plunges at a motion frequency that is lower than the natural frequency of the system and backward if the frequency ratio, the ratio between the motion frequency and the natural frequency, is greater than one. Similarly, Masoud and Alexeev [28] used the lattice Boltzmann method to show that at the frequency ratio of 0.95 the maximal propulsive force was obtained. The magnitude of the maximal force would increase when the inertial effects became more important than the fluid inertia. Michelin and Llewellyn Smith [30] used potential flow theory to describe the flow over a plunging flexible wing. The trailing-edge flapping amplitude and the propulsive force are shown to be maximal at resonance conditions. In a series of experiments using self-propelled simplified insect model, Thiria and Godoy-Diana [25] and Ramananarivo, Godoy-Diana, and Thiria [26] also show that the maximum thrust force was around frequency ratio of 0.7. More recently, Gogulapati and Friedmann [29] coupled an approximate aerodynamic model, which was extended to forward flight including the effects of fluid viscosity, to a nonlinear structural dynamic model. For various setups of composite anisotropic Zimmerman wings [31], they investigated the propulsive force generation in forward flight. The maximum propulsive force was also obtained at the frequency ratio slight lower than one. These observations are consistent with the general perception of the resonance phenomena in which even small external force can induce large amplitude deformations and potentially be efficient as well.

However, it was reported for insects, that the flapping frequency of the insects is below the natural frequencies of the wing, only a fraction of the resonance frequency [32,33]. Sunada, Zeng, and Kawachi [32] measured the natural frequencies of vibration in air and the wing beat frequencies for four different dragonfly wings. The wing beat frequency ratio were in the range of $0.30-0.46$. Chen, Chen, and Chou [33] have also measured the wing beat frequencies and natural frequencies of the dragonfly wings. In their measurements the average flapping frequency was $27 \mathrm{~Hz}$ while the natural frequency, calculated using a spectrum analyzer, was $170 \mathrm{~Hz}$ when it is clamped at the wing base, resulting in a frequency ratio of about 0.16 . The propulsive efficiency was also investigated numerically [34,28] and experimentally using a self-propelled model [25,26]. Vanella et al. [34] conducted numerical investigations on a two link model and found that the optimal performance as realized when the wing was excited at the frequency ratio of 0.33 . For all Reynolds numbers considered in the range of 75 to 1000 the wake capture mechanism was enhanced due to a stronger flow around the wing at stroke reversal, resulting from a stronger vortex at the trailing edge. Thiria and Godoy-Diana [25] and Ramananarivo, Godoy-Diana, and Thiria [26] also showed using the experimental setup that was described above that the maximum efficiency was obtained at a frequency ratio lower than that of the maximum propulsion at 0.7 . They concluded that the performance optimization is not by looking at the resonance, but adjusting the temporal evolution of the wing shape. On the other hand, Masoud and Alexeev [28] showed that the optimal efficiency for a hovering flat plate at $R e=100$ was when the motion was excited at the frequency ratio of 1.25 . In their setup the flexible flat plate has a geometric angle of attack of 40 degrees in contrast to the previously mentioned studies where the plunging motion was symmetric.

The objective of the this study is three-fold: i) Provide a framework to analyze the effect of flexibility on flapping wings; ii) Elucidate the interplay between the fluid dynamic forces on the wing and the scaling parameters; and iii) Understand the underlying physics of force generation and propulsive efficiency of flapping wings related to the wing flexibility. First, starting from the Navier-Stokes equations the forces acting on a moving body immersed in a fluid, such as a wing in air or water, will be scaled by properly normalizing the equations. The concepts such as added mass effects, that denotes the force on the wing that is proportional to the wing acceleration and the hydrodynamic impulse will be introduced. The analysis of the fluid dynamic performance of flexible wings is similar to that of flutter in the aeroelasticity community: in flutter research the wing oscillations are the results of the interaction between the wing deformations and the aerodynamic loading, here the kinematic motion at the root of the wing is given and we look for the response of the wing structures, the aerodynamics, and the interaction between these two. To quantify the interplay of the flapping kinematics and the response of the flexible wing structure and to identify the dominant parameter involved, three canonical cases with sinusoidal kinematics will be considered to assess the role of the chordwise flexible, spanwise flexible, and isotropic wings on the resulting fluid dynamic 
forces. Based on these canonical cases, we will identify the underlying physical mechanism and propose a scaling parameter for the force generation and the propulsive efficiency of flexible flapping wings that depends on the $a$ priori known non-dimensional parameters. Identifying this scaling parameter leads to an order of magnitude estimation of the flexilibity enhanced fluid dynamic performance. Furthermore, we will extrapolate the scaling parameter to the insects by assuming that the weight of the insects are sustained by the lift and show that the generated lift also follows the same scaling.

The outline of this paper is as follows. First, a dimensional analysis will be performed in Section II, followed by the non-dimensionalization of the governing equations. In Section III, the numerical framework for flexible flapping wings will be introduced. Then in Section IV, the scaling of the forces will be discussed based on a control volume analysis. In Section V the scaling relationships for the force generation and the propulsive efficiency for flexible flapping wings will be established for high Reynolds number and high reduced frequency systems. Based on the numerical computations of i) the thrust generation of a plunging chordwise flexible airfoil in Secion V.1.A, ii) spanwise flexible wing in Section V.1.B in forward flight in water for different wing stiffnesses and motion frequencies, the physics of fluid-structure interaction between the plunging wing and the fluid flow will be explored. Furthermore, the lift generation of a flapping isotropic Zimmerman wing in hover in air (Section V.1.C) will be shown to follow a similar mechanism. For this case a surrogate model will be constructed based on the variations of Young's modulus and wing density. Furthermore, based the identified mechanism, a scaling parameter for the force generation due to the flexibility of the wing will be proposed using a dynamic beam analysis in Section V.1.D, with an extrapolation of the scaling to insects. Finally, a scaling parameter will be obtained for the propulsive efficiency in Section V.2 with a discussion on the applicability of the scaling on the MAV design and interpretation of the observed physics.

\section{Dimensional Analysis and Non-Dimensional Governing Equations}

The relevant physical quantities related to the system of flexible flapping wing fluid dynamics are the density, $\rho_{f}$ and the viscosity, $\mu$, of the fluid; the reference velocity, $U_{\text {ref }}$, of the fluid flow field; the half span, $R$, the mean chord, $c_{\mathrm{m}}$, and the thickness, $h_{s}$, of the wing geometry; the density, $\rho_{s}$ and the Young's modulus, $E$, and the Poisson's ratio, $v$, of the wing structure; the flapping (plunging) amplitude, $\phi_{a}\left(h_{a}\right)$, the flapping frequency, $\omega /(2 \pi)$, and the geometric angle of attack, $\alpha$; and finally the resulting aerodynamic force, $F$, see Figure II- 1 . There are 13 variables and three fundamental dimensions leading to 10 non-dimensional parameters. With $\rho_{f}, U_{\text {ref }}$, and $c_{\mathrm{m}}$ as the basis variables to independently span the fundamental dimensions, the dimensional analysis leads to the non-dimensional parameters shown in Table II-1. The resulting set of non-dimensional parameters consists of most of the well-known parameters in the flapping wing aerodynamics community, however it is not unique.

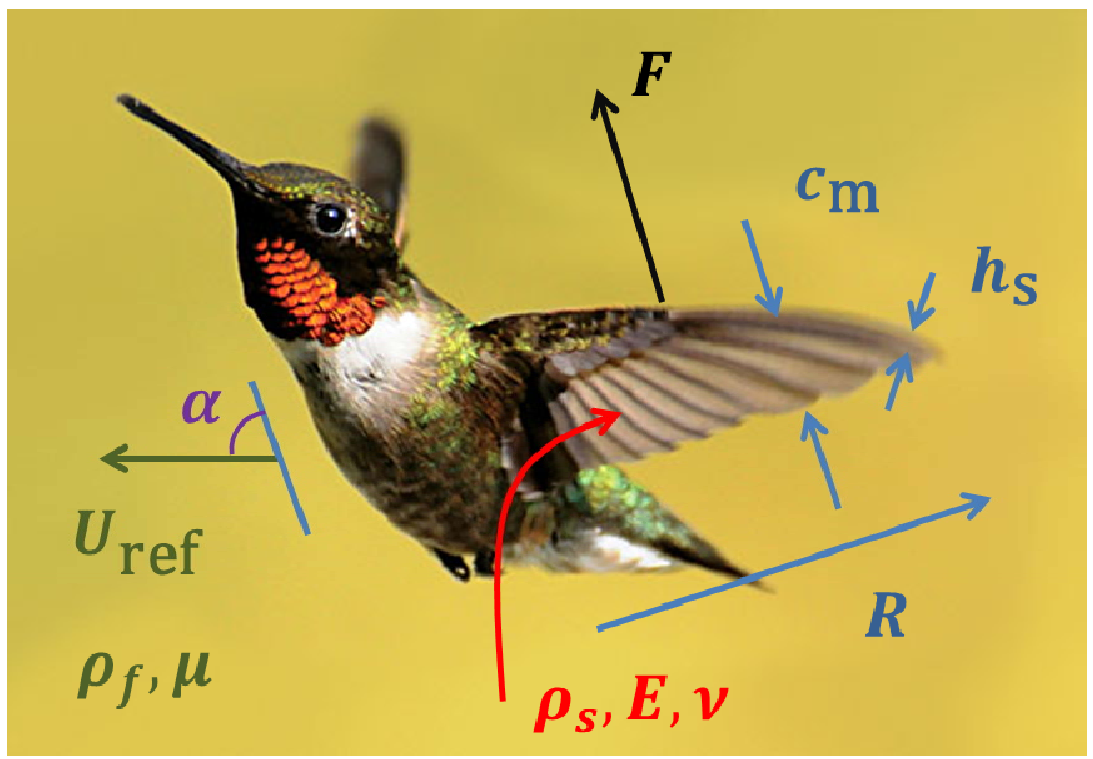

Figure II-1 Relevant physical variables shown for a case of hummingbird. The picture is reproduced, by permission, from Wei Shyy (c) [35]. 
The governing equations are non-dimensionalized with the reference velocity, $U_{\text {ref }}$, as the velocity scale, inverse of the motion frequency, $2 \pi / \omega$ as the time scale, and the mean chord, $c_{\mathrm{m}}$, as the length scale. For forward flight cases the forward velocity of the wing, i.e. the incoming velocity in the frame of reference of the wing, is chosen as $U_{\text {ref }}$ and for hovering flight the mean wing tip velocity during half stroke is taken as the reference velocity. The resulting governing equations for the incompressible fluid modeled by the unsteady three-dimensional NavierStokes equations with constant density and viscosity are

$$
\begin{gathered}
\frac{\partial}{\partial x_{i}^{*}}\left(u_{i}^{*}\right)=0 \\
\frac{k}{\pi} \frac{\partial}{\partial t}\left(u_{i}^{*}\right)+\frac{\partial}{\partial x_{i}^{*}}\left(u_{j}^{*} u_{i}^{*}\right)=-\frac{\partial p^{*}}{\partial x_{i}^{*}}+\frac{1}{\operatorname{Re}} \frac{\partial}{\partial x_{j}^{*}}\left\{\frac{\partial u_{i}^{*}}{\partial x_{j}^{*}}\right\}
\end{gathered}
$$

where $(\cdot)^{*}$ indicates non-dimensional variables. The reduced frequency $k$ is a measure of unsteadiness by comparing the spatial wavelength of the flow disturbance to the chord [36]. For hover, the reference velocity is based on the mean wing tip velocity, thus reduced frequency is inversely proportional to the flapping (plunging) amplitude and aspect ratio of the wing and is not related to the flapping frequency. On the other hand, the reduced frequency based on the forward speed is proportional to flapping frequency and the mean chord length, and inversely proportional to the flight speed. Another interpretation of the reduced frequency is that it gives the ratio between the fluid convection time scale, $c_{\mathrm{m}} / U_{\text {ref }}$, and the motion time scale, $2 \pi / \omega$. The Reynolds number $R e$ is the ratio between the inertial and the viscous forces in the fluids. For hover the Reynolds number is proportional to the flapping (plunging) amplitude, the flapping frequency, square of the mean chord length, and the aspect ratio of the wing.

Table II-1 Non-dimensional parameters for the flexible flapping wing systems

\begin{tabular}{cccc}
\hline \hline Non-dimensional parameter & Symbol & Definition & Note \\
\hline Reynolds number & $R e$ & $\rho_{f} U_{\mathrm{ref}} c_{\mathrm{m}} / \mu$ & \\
Aspect ratio & $A R$ & $R / c_{\mathrm{m}}$ & \\
Thickness ratio & $h_{s}^{*}$ & $h_{s} / c_{\mathrm{m}}$ & \\
Density ratio & $\rho^{*}$ & $\nu$ & plate \\
Poisson's ratio & $v$ & $E h_{s}^{* 3} /\left\{12\left(1-\rho^{2}\right) \rho_{f} U_{\mathrm{ref}}^{2}\right\}$ & beam \\
Effective stiffness & $\Pi_{1}$ & $E h_{s}^{* 3} /\left\{12 \rho_{f} U_{\mathrm{ref}}^{2}\right\}$ & flapping \\
Reduced frequency & $k$ & $\omega c_{\mathrm{m}} /\left(2 U_{\mathrm{ref}}\right)$ & plunging \\
Strouhal number & $S t$ & $\phi_{a} A R k / \pi$ & plunging \\
Effective angle of attack & $\alpha_{e}$ & $\alpha+\operatorname{atan}(2 \pi S t)$ & \\
Force coefficient & $C_{F}$ & $F /\left(\frac{1}{2} \rho_{f} U_{\mathrm{ref}}^{2} c_{\mathrm{m}}^{2} A R\right)$ & \\
\hline
\end{tabular}

The flexible wing structure is modeled locally by

$$
\rho^{*} h_{s}^{*}\left(\frac{k}{\pi}\right)^{2}\left(\frac{L}{c_{\mathrm{m}}}\right) \frac{\partial^{2} w^{*}}{\partial t^{* 2}}+\Pi_{1}\left(\frac{c_{\mathrm{m}}}{L}\right)^{3} \Delta^{* 2} w^{*}=f_{f}^{*},
$$

where $w$ is the displacement due to bending motion, $\Delta^{*}=\partial^{* 2} / \partial x_{i}^{* 2}$ the Laplacian operator, and $f_{f}^{*}$ the distributed transverse fluid force on the wing per unit span. A special care is given in the direction of the wing bending, because the correct length-scale for the spanwise bending is the half span $R$ and not the chord $c_{\mathrm{m}}$. The correction factor that arises is expressed as $\left(L / c_{\mathrm{m}}\right)$, where $L / c_{\mathrm{m}}=1$ for the chordwise flexible airfoil case (Section V.1.A), and $L / c_{\mathrm{m}}=$ $A R$ for the spanwise flexible wing case (Section V.1.B) and isotropic Zimmerman wing case (Section V.1.C), where $A R$ is the aspect ratio of the wing: For the three-dimensional wings the bending motion is aligned with $R$, so that a factor of $A R$ is required to renormalize the transverse displacement. The density ratio $\rho^{*}$ is the ratio between the wing density and the fluid density and the effective stiffness $\Pi_{1}[6]$ gives the ratio between the elastic bending forces and the fluid dynamic forces. The coefficient of the inertial term will be used frequently later in the discussion of the 
fluid dynamic performance of flexible wings in Section $\mathrm{V}$ and is abbreviated as the effective inertia $\Pi_{0}=$ $\rho^{*} h_{s}^{*}(k / \pi)^{2}$.In this study the prescribed motion at either the root of the wing or the region of the wing specified is sinusoidal of the form

$$
\phi\left(t^{*}\right)=\frac{S t}{A R} \frac{\pi}{k} \sin \left(2 \pi t^{*}\right)
$$

for the flapping motion and

$$
h\left(t^{*}\right)=S t \frac{\pi}{k} \sin \left(2 \pi t^{*}\right)
$$

for the plunging motion, where the sine function can be replaced by the cosine function. The Strouhal number $S t$ appears in combination with $k$ to give the plunge amplitude $h_{a} / c_{\mathrm{m}}$. The Strouhal number indicates the ratio between the flapping speed and the reference velocity. It characterizes the vortex dynamics of the wake and shedding behavior of vortices of a flapping wing in forward flight $[3,36]$. Still unclear is the meaning and the role of the Strouhal number in hover, since for hover the Strouhal number will reduce to a constant. Active pitching motion is not considered in this paper.

Finally, the force coefficient is then given by a to-be-determined relation

$$
C_{F}=\widetilde{\Psi}\left(R e, A R, h_{s}^{*}, \rho^{*}, \Pi_{1}, k, S t\right) .
$$

In this study the aim is to simplify Eq. (5) by reducing the number of non-dimensional parameters involved. The time-averaged force $\left\langle C_{F}\right\rangle$ of $C_{F}$ are calculated as

$$
\left\langle C_{F}\right\rangle=\frac{\omega}{2 \pi} \int_{2 \pi m / \omega}^{2 \pi(m+1) / \omega} C_{F} d t,
$$

where $m=2$ unless otherwise specified. The reason for setting $m>1$ is to avoid initial transient effects.

\section{Numerical Methods}

\section{III.1 Fluid Dynamics Solver}

The governing equations for the fluids given by Eq. (1) are solved with Loci-STREAM $[37,38,39]$, which is a three-dimensional, unstructured, pressure-based finite volume solver written in the Loci-framework. It employs implicit first or second order time stepping and treats the convection terms using the second order upwind-type scheme and the pressure and viscous terms using second order schemes. The system of equations resulting from the linearized momentum equations are handled with the symmetric Gauss-Seidel solver. The pressure correction equation is solved with either the GMRES linear solver with Jacobi preconditioner provided by PETSc [40,41,42], or the BoomerAMG [43] linear solver provided by hypre. The Loci-framework is by design rule-based highly parallelizable framework for finite volume methods [44]. The geometric conservation law [45], a necessary consideration in domains with moving boundaries, is satisfied [46]. The mesh deformations are realized using radial basis function (RBF) interpolations [47]. The turbulence closure is modeled by Menter's Shear Stress Transport turbulence model $[48,49]$.

\section{III.2 Structural Dynamics Solvers}

Two structural dynamics solvers with different fidelity have been incorporated. For linear analysis of a beamlike flat plate an Euler-Bernoulli beam model has been incorporated to solve Eq. (2) in one-dimension, i.e. $\Delta=\partial^{2} / \partial x^{2}$. To simulate large displacement wing motions geometrically nonlinear structural dynamics equations are modeled with a flexible multi-body type finite element using triangular shell elements. The rigid-body motions are prescribed in the global frame of reference in addition to a co-rotational framework to account for the geometric nonlinearities. By applying the co-rotational frame transformations the motion of an element is decomposed into the rigid-body motion part and the pure deformation part. By using linear elasticity theory for the latter, the co-rotational 
formulation can efficiently solve for the structural dynamics with small strains, yet large rotations. A linear combination of an optimal membrane element and a discrete Kirchhoff triangle plate bending element is employed for the elastic stiffness of a shell element [50,51].

\section{III.3 Fluid-Structure Interaction Interface}

The fluid-structure interaction (FSI) is based on a time-domain partitioned solution process in which the nonlinear partial differential equation governing the fluid and the structure are solved independently and spatially coupled through the interface between the fluid and the structure. An interface module has been added to the fluid solver to communicate the parallelized flow solutions on the three-dimensional wetted surface to and from the serial structural solver. At each time step the fluid and the structural solvers are called one after the other until sufficient convergence on the displacements on the shared boundary surface are reached in an inner-iteration before advancing to the next time step. Full details of this algorithm are described in Ref. [52]. In this study, in order to accelerate and ensure the convergence of the FSI the Aitken relaxation method [53] has been incorporated. The numerical implementation of the Aitken relaxation method in the current numerical framework is described in Ref. [54].

\section{Scaling of the Forces Acting on a Moving Body Immersed in Fluid}

\section{IV.1 Scaling of Forces}

Consider a control volume of fluid domain $V_{\infty}$ bounded by $S_{\infty}$ at infinity in which a body $\Omega$ moves in time $t$ and space $x \in V$. Integration of Eq. (1) in $V_{\infty}$ yields,

$$
\begin{aligned}
\frac{d}{d t} \int_{V_{\infty}(t)} \rho_{f} \boldsymbol{u} d V & +\int_{\partial \Omega(t)} \boldsymbol{n} \cdot(-p \mathbf{I}+\mathbf{T}) d S \\
& =\int_{S_{\infty}} \boldsymbol{n} \cdot(-p \mathbf{I}+\mathbf{T}) d S-\int_{S_{\infty}} \boldsymbol{n} \cdot\left(\boldsymbol{u}-\boldsymbol{u}_{s}\right) \rho_{f} \boldsymbol{u} d S \\
& -\int_{\partial \Omega(t)} \boldsymbol{n} \cdot\left(\boldsymbol{u}-\boldsymbol{u}_{s}\right) \rho_{f} \boldsymbol{u} d S
\end{aligned}
$$

where $\partial \Omega(t)$ is the boundary surface of $\Omega, \boldsymbol{n}$ the unit normal pointing outward from the body, $\boldsymbol{u}_{s}$ is the velocity of the surface, $\mathbf{I}$ the unit tensor, and $\mathbf{T}$ the viscous stress tensor.

The force $\boldsymbol{F}$ acting on a moving body $\Omega$ immersed in an incompressible viscous fluid is

$$
\boldsymbol{F}=\rho_{f} \int_{\partial \Omega(t)} \boldsymbol{n} \cdot(-p \mathbf{I}+\mathbf{T}) d S
$$

With no-flow through boundary condition, $\left(\boldsymbol{u}-\boldsymbol{u}_{s}\right) \cdot \boldsymbol{n}=0$ on $\partial \Omega$ and under the assumption that the viscous terms and convective terms are negligible at $S_{\infty}$, Eq. (7) simplifies to

$$
\boldsymbol{F}=-\frac{d}{d t} \int_{V_{\infty}(t)} \rho_{f} \boldsymbol{u} d V+\int_{S_{\infty}} \boldsymbol{n} \cdot(-p \mathbf{I}) d S-\int_{\partial \Omega(t)} \boldsymbol{n} \cdot\left(\boldsymbol{u}-\boldsymbol{u}_{S}\right) \rho_{f} \boldsymbol{u} d S
$$

Based on Eq. (9), Noca [55] derived an expression for $\boldsymbol{F}$ for a doubly connected infinite fluid domain $V_{\infty}$ as

$$
\frac{\boldsymbol{F}}{\rho_{f}}=-\frac{1}{\mathcal{N}-1}\left(\boldsymbol{F}_{\mathrm{im}}+\boldsymbol{F}_{\mathrm{a}}\right)
$$

where

$$
\boldsymbol{F}_{\mathrm{im}}=\frac{d}{d t} \int_{\mathrm{V}_{\infty}} \boldsymbol{x} \times \xi d V
$$




$$
\boldsymbol{F}_{\mathrm{a}}=\frac{d}{d t} \int_{\partial \Omega(t)} \boldsymbol{x} \times(\boldsymbol{n} \times \boldsymbol{u}) d S
$$

where $\mathcal{N}$ is the spatial dimension and $\xi=\nabla \times \boldsymbol{u}$ is the vorticity. The first integral, $\boldsymbol{F}_{\text {im }}$, represents the time derivative of the hydrodynamic impulse, see e.g. [56], which is equal to the non-conservative external body forces for inviscid flows. Following the discussion given by Saffman [56], consider a three-dimensional flow field resulting from a motion of a moving body, such that the vortices are confined in a material volume, $V_{\mathrm{v}}$. Then we have for the impulse

$$
\boldsymbol{F}_{\mathrm{im}} \approx \frac{d}{d t} \int_{V_{\mathrm{v}}} \boldsymbol{x} \times \xi d V_{\mathrm{v}}=\int_{V_{\mathrm{v}}} \boldsymbol{x} \times \frac{D \xi}{D t}+\boldsymbol{u} \times \xi d V_{\mathrm{v}}=2 \int_{V_{\mathrm{v}}} \frac{\mu}{\rho_{f}} \boldsymbol{x} \times \nabla^{2} \xi d V_{\mathrm{v}}+2 \int_{V_{\mathrm{v}}} \boldsymbol{u}_{\mathrm{e}} \times \xi d V_{\mathrm{v}}
$$

where the external velocity $\boldsymbol{u}_{\mathrm{e}}$ is due to other vortices not confined in $V_{\mathrm{v}}$ or the motion of the body. Because of the time evolution of the vorticity in the flow field, the force due to this term may have different phase than the motion of the body. Saffman [56] also proves that for inviscid, irrotational flow without net circulation around $\Omega$ the second integral in Eq. (10), $\boldsymbol{F}_{\mathrm{a}}$, represents the force due to added mass. Noca [55] further shows that for an impulsively starting flat plate in a viscous flow with no-slip boundary condition on $\Omega$ in quiescent fluid the force acting on the body has the same expression immediately after the start of the motion.

Using the non-dimensionalization procedure introduced in Section II the magnitudes of the force components in Eq. (10) are estimated as follows. For the hydrodynamic impulse term, if we assume that the vorticity is confined in a rectangular fluid region in the order of $O\left(c_{\mathrm{m}}\right)$ in the streamwise direction and $O\left(h_{a}\right)$ in the normal direction and that $x \sim c_{\mathrm{m}}, \xi \sim U_{\mathrm{ref}} / c_{\mathrm{m}}$, and $u_{\mathrm{e}} \sim \omega h_{a}$ in the integrand Eq. (13),

$$
C_{F, \mathrm{im}} \sim S t\left\{\frac{1}{\operatorname{Re} k} O(1)+\frac{S t}{k} O(1)\right\} .
$$

as a first order approximation. Hence the force due to the hydrodynamic impulse scales with $S t$, however if the viscous time scale, $c_{\mathrm{m}}^{2} / v$, is much greater than the motion time scale, $1 / \omega$, such that $R e k \gg 1$, then the first term in Eq. (14) becomes negligible. Moreover, when the plunge amplitude $h_{a} / c_{\mathrm{m}} \sim S t / k$ is small the second term in Eq. (14) will only have a small contribution to the total force felt on the wing. In general, however, complex fluid dynamics mechanisms, such as the wing-wake interaction, or the wake-wake interactions would additionally affect the vorticity distribution in the flow field.

Similarly, the force due to added mass can be non-dimensionalized as

$$
C_{F, \mathrm{a}} \sim S t k
$$

with $u \sim \omega h_{a}$ in the integrand of Eq. (12). This non-dimensionalization process reveals that the fluid dynamic force is proportional to $S t$, hence with increasing $S t$ the force acting on the wing is expected to increase On the other hand, if the motion is highly unsteady, i.e. $k$ is high, the force due to the motion of the body appearing as the added mass component, dominates over the forces due to vorticity in the flow field.

A parametrization of special interest for flapping wing community is the dependence of the force on the flapping motion frequency, $\omega$. The current scaling shows that for forward flight with $U_{\text {ref }}=U_{\infty}$ the added mass has the largest order of frequency as $\sim \omega^{2}$. The resulting dimensional force is then proportional to square of the motion frequency. Similarly, for hovering motions the current scaling shows that the non-dimensional force is independent of the motion frequency since the Strouhal number is a constant and the reduced frequency only a function of flapping (plunging) amplitude. However, since $U_{\text {ref }} \sim \omega^{2}$ the resulting dimensional force is also proportional to square of the motion frequency. Similar observations were reported by Gogulapati and Friedmann [29] who conducted potential theory based aerodynamic analysis of flappingwings in hovering and forward flight.

At high Reynolds number, high reduced frequency regime, Visbal, Gordnier, and Galbraith [57] considered a high frequency small amplitude plunging motion at $R e=O\left(10^{4}\right)$ over a three-dimensional SD7003 wing $\left(\alpha_{0}=4\right.$ deg; $k=3.93 ; S t=0.06 ; R e=1 \times 10^{4}, 4 \times 10^{4}$ ). They used the iLES (implicit Large Eddy Simulation) approach to solve for the flow structures including the laminar-to-turbulence transition and the forces on the wing. The flow field exhibits formation of dynamic-stall like leading edge vortices, breakdown due to spanwise instabilities, and transitional features, however the forces on the wing could still be well predicted by the Theodorsen Eq. (16) 
formula for lift.

$$
\begin{aligned}
& C_{L}=2 \pi\{1-C(k)\} \alpha_{0} \\
& +\pi k\left\{\alpha_{a} i e^{\psi i}-2 \pi S t-\alpha_{a} k\left(2 x_{p}-1\right) e^{\psi i}\right\} e^{2 \pi t^{*} i} \\
& +2 \pi C(k)\left\{2 \pi S t i+\alpha_{a} e^{\psi i}+\alpha_{a}\left(\frac{3}{2}-2 x_{p}\right) k i e^{\psi i}\right\} e^{2 \pi t^{*} i}
\end{aligned}
$$

The time history of lift was "independent of Reynolds number and of the 3-D transitional aspects of the flow field" [57]. They explained that the lift is dominated by the acceleration of the airfoil, which is proportional to the square of the motion frequency. This observation is also consistent with the scaling that the hydrodynamic impulse term Eq. (11) is small compared to the added mass term Eq. (12) for the given non-dimensional parameters.

On the other hand, at lower Reynolds numbers, Trizila et al. [58] have shown at $R e=100$ and $k$ in the range of $0.25-0.5$ that the formation and interaction of leading edge and trailing edge vortices with the airfoil and previous shed wake substantially affect the lift and power generation for hover and forward flight. Furthermore, threedimensionality effects play a significant role, for instance for a delayed rotation kinematics $(k=0.5$; low angle of attack; $R e=100)$ the tip vortex generated at the tip of the $A R=4$ flat plate would interact with the leading edge vortex enhancing lift compared to its two-dimensional counterpart, which contrasts the classical steady-state thin wing theory [58], [59] which predicts the formation of wing tip vortices as lift reducing flow feature. This complex interplay between the kinematics, the wing-wake, wake-wake interactions, and the fluid dynamic forces on the wing at the given range of non-dimensional parameters is also consistent with the scaling analysis described in this Section.

\section{IV.2 Linearized Aerodynamic Theories}

When a body accelerates in a fluid, the fluid kinetic energy changes. The rate of work done by pressure moving the body in an inviscid fluid yields a force that is proportional to the acceleration (see e.g. [60]). Then, the constant of proportionality has the dimension of mass, hence the name added mass. The added mass term is usually some fraction of the fluid mass displaced by the body. Determination of the added mass, which is a tensor, because it relates the acceleration vector to the force vector, is not easy in general because the local acceleration of the fluid is not necessarily the same as the acceleration of the body [61]. However, for an accelerating thin flat plate with a chord length of $c_{\mathrm{m}}$ normal to itself, the force acting normal to the flat plate, can be obtained as follows. The velocity potential difference is (e.g. [61]),

$$
\Delta \phi=c_{\mathrm{m}} v_{i} \sqrt{1-\left(\frac{x}{c_{\mathrm{m}} / 2}\right)^{2}}
$$

where $v_{i}$ is the vertical velocity component. Hence the vertical component of the force acting on the flat plate due to added mass becomes,

$$
\rho_{f} F_{\mathrm{a}}=\frac{d}{d t}\left(\rho_{f} \frac{\pi}{4} v_{i} c_{\mathrm{m}}^{2}\right)
$$

whence the added mass of a vertically accelerating thin flat plate is equal to the displaced fluid cylinder with radius $c_{\mathrm{m}} / 2$.

For a harmonically plunging thin rigid flat plate in a freestream the lift coefficient can be derived assuming inviscid incompressible flow as

$$
C_{L}=2 \pi^{2} S t k \cos (\omega t)+4 \pi^{2} S t \sin (\omega t)
$$

assuming quasi steady-state flow where the influence of the wake vorticities are neglected. The first term in Eq. (18) is the non-circulatory term which is consistent with the added mass force derived in Eq. (12). The second term in Eq. (18) is the circulatory term, which can be expressed in a more familiar form, $2 \pi \alpha_{\mathrm{e}}$, by recognizing that $2 \pi S t \sin (\omega t) \approx \alpha_{\mathrm{e}}$ where $\alpha_{\mathrm{e}}$ is the effective angle of attack for purely plunging motions. Both terms are also consistent with the scaling found in Eq. (14) and Eq. (15).

A more accurate representation of the lift coefficient beyond the quasi-steady approximation was Theodorsen 
[62], for sinusoidal pitch-plunge of a thin airfoil, by assuming a planar wake and a trailing-edge Kutta condition, in incompressible inviscid flow, see Eq. (16). The pitch and plunge motions are described by the complex exponentials, $\alpha\left(t^{*}\right)=\alpha_{0}+\alpha_{a} e^{\left(2 \pi t^{*}+\psi\right) i}$ and $h\left(t^{*}\right)=S t \pi / k e^{2 \pi t^{*} i}$. The phase lead of pitch compared to plunge is denoted by $\psi$. In the most common case, motivated by considerations of maximum propulsive efficiency, pitch leads plunge by $90^{\circ}$, which results in $\psi=\pi / 2$ [63]. The $C(k)$ is the complex-valued Theodorsen function with magnitude $\leq 1$. It accounts for attenuation of lift amplitude and time-lag in lift response, from its real and imaginary parts, respectively. The first term is the steady-state lift and the second term is the noncirculatory lift due to acceleration effects. The third term models circulatory effects. Setting $C(k)=1$ recovers the quasi-steady thin airfoil solution. Note that $C(k)=1$ for pure plunge kinematics with $\alpha_{0}=\alpha_{a}=0$ yields Eq. (18).

The scaling for the hydrodynamic impulse and the added mass terms are summarized in Table IV-1. The scaling shows that for low reduced frequency motions or low Reynolds number flows the hydrodynamic impulse term, which indicates the interaction between the vortices and the wing becomes important. On the other hand, when the reduced frequency increases the added mass term will dominate over the hydrodynamic impulse term. Both components are proportional to the Strouhal number.

Table IV-1 Summary of the force scaling

\begin{tabular}{|c|c|c|}
\hline Force & Scaling & Note \\
\hline \multirow{2}{*}{ Hydrodynamic impulse } & $S t /(\operatorname{Re} k) \sim \omega^{0}$ & viscous term \\
\hline & $S t^{2} / k \sim \omega^{1}$ & vortex force due to motion of moving body \\
\hline Added mass & $S t k \sim \omega^{2}$ & \\
\hline
\end{tabular}

An interesting consequence that needs to be investigated more is that for hovering flight condition where both Strouhal number and reduced frequencies are independent of motion frequency, the normalized force will be independent of frequency, for high Reynolds number flows.

\section{Effects of Flexibility on the Fluid Dynamic Performance of Wings}

In this section the effects of flexibility on the fluid dynamic performances, i.e. propulsive force generation and the propulsive efficiency, are considered. The findings reported in the literature can be summarized by the following two observations:

(i) Maximum propulsive force is generated when the wing motion frequency is near the natural frequency of the wing $[27,28,25,26,29,30]$.

(ii) Optimal propulsive efficiency is obtained at a motion frequency that is only a fraction of the natural frequency $[34,28,25,26]$.

To probe and to elucidate the mechanisms related to these observations, first three canonical cases with sinusoidal kinematics will be considered to assess the role of the chordwise flexibility, spanwise flexibility, and isotropic wings on the resulting fluid dynamic forces: i) thrust generation of a purely plunging chordwise flexible airfoils in water in forward flight at $R e=9.0 \times 10^{3}$, studied experimentally by Heathoote and Gursul [64] and numerically for one particular motion frequency by Shyy et al. [6]. The airfoil consists of rigid teardrop leading edge with elastic flat plate as tail. In this study five flat plate thicknesses will be considered for various motion frequencies with resulting thrust; ii) thrust generation of a purely plunging spanwise flexible wing with NACA0012 airfoil in water in forward flight at $R e=3.0 \times 10^{4}$ considered by Heathcote, Wang, and Gursul [65], Chimakurthi et al. [52], and Shyy et al. [6]. Two different wing materials will be used to evaluate the wing flexibility effects on the resulting thrust for a range of motion parameters; iii) lift generation of flapping isotropic Zimmerman wing hovering in air at $R e=1.5 \times 10^{3}$ and $k=0.56$ where the Young's modulus and the mass of the wing are varied to assess the effects of the structural properties on the resulting lift. Although a wing with the same geometry is preferred to study the effects of the chordwise and the spanwise flexibilities independently, the first two cases studied in this study are chosen, because the experimental results [64] [65] are well documented for validation purposes. The design space of the third case is based on several choices of the wing material and fluids that are used in wind/water tunnel experiments as well as Micro Air Vehicle (MAV) applications. Based on these canonical cases, the underlying physical mechanisms will be identified and a scaling parameter for the force generation and the propulsive efficiency of flexible flapping wings will be proposed that depends on the a priori known non-dimensional parameters. Identifying this scaling parameter leads to an order of magnitude estimation of the flexibility enhanced fluid dynamic performance. Furthermore, the scaling parameter will be extrapolated to the case of insects by 
assuming that the weight of the insects are sustained by the lift and it will be shown that the lift generated also follows the same scaling. Finally, scaling for the propulsive efficiency will be presented that sheds light on the two observations mentioned at the beginning of this section.

\section{V.1 Propulsive Force Generation of Flapping Flexible Wings}

\section{V.1.A Purely Plunging Chordwise Flexible Airfoil in Forward Flight in Water}

To explore the thrust enhancement induced by chordwise flexibility, the thrust of a purely plunging chordwise flexible airfoil is computed for different thickness ratios $\left(h_{s}^{*}=4.23 \times 10^{-3}, 1.41 \times 10^{-3}, 1.13 \times 10^{-3}, 0.85 \times 10^{-3}\right.$, and $0.56 \times 10^{-3}$ ) and motion frequencies that produce Strouhal numbers between $S t=0.085$ and 0.3 with 0.025 increment with the plunge amplitude kept fixed to $h_{\mathrm{a}} / c_{\mathrm{m}}=0.194$. The reduced frequency $k$ varies then between 1.4 and 4.86 . As shown in Table II-1 variation in the thickness changes $\Pi_{1}$, whereas the motion frequency affects both the reduced frequency and the Strouhal number. The airfoil consists of a rigid teardrop leading edge and an elastic plate that plunges sinusoidally in freestream. Detailed experimental setup and discussion of fluid physics is in Ref. [64], and Shyy et al. [6] obtained a numerical solution for $S t=0.17$ for different thickness ratios. An Euler-Bernoulli beam solver is used to solve Eq. (2) for the deformation of the elastic flat plate, while the rigid teardrop moves with the imposed kinematics. Furthermore, the Reynolds number $R e=9.0 \times 10^{3}$ and the density ratio $\rho^{*}=7.8$ are held constant in all cases. The grid and time step sensitivity studies are shown in Appendix A.1.

To validate the current computation to the experimental measurements [64] the trailing edge motion is plotted as function of $t / T$ for the moderate thick $h_{s}^{*}=1.41 \times 10^{-3}$ and the thinnest $h_{s}^{*}=0.56 \times 10^{-3}$ cases at $S t=0.17$ in Figure $\mathrm{V}-1$. For the thinnest airfoil the displacement peak in the downstroke near $t / T=0.25$ is slightly overpredicted, however the overall trend matches well. At $S t=0.17$ the thinnest airfoil deformes considerably more than the airfoil with $h_{S}^{*}=1.41 \times 10^{-3}$ with larger phase lag.

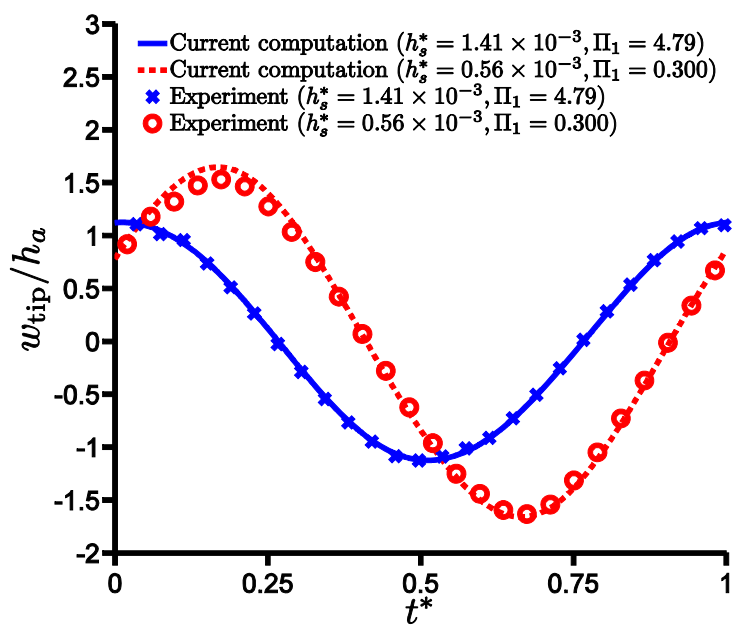

Figure V-1 Trailing edge displacement as function of non-dimensional time for the purely plunging chordwise flexible airfoils in water in freestream at $R e=9.0 \times 10^{3}$ with $h_{s}^{*}=1.41 \times 10^{-3}$ and $0.56 \times 10^{-3}$ at $S t=0.17$. The experimental measurements are from [64].

For $S t=0.17$ the thrust coefficient as a function of normalized time (with respect to the period of plunge) for the Rigid $\left(h_{s}^{*}=4.23 \times 10^{-3}\right)$, Flexible $\left(h_{s}^{*}=1.41 \times 10^{-3}\right)$, and Very Flexible $\left(h_{s}^{*}=0.56 \times 10^{-3}\right)$ thickness ratios is shown in Figure V-2. In order to estimate the individual contribution of the teardrop and the flexible plate to force generation, the time histories of thrust coefficient are shown in Figure V-2 separately for each element. It is seen that the thrust response with variation in flexibility is different in each of the two cases: with increasing chordwise flexibility of the plate the instantaneous thrust contributed by the flexible plate increases. One of the mechanisms found in Ref. [6] is that the chordwise deformation of the rear flexible plate in both Flexible and Very Flexible cases result in an effective projected area for the thrust forces to develop. 


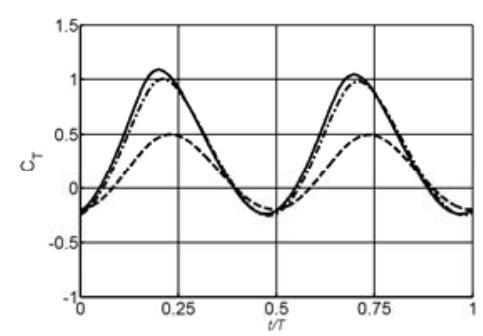

(a) $C_{T}$ generated by the rigid teardrop

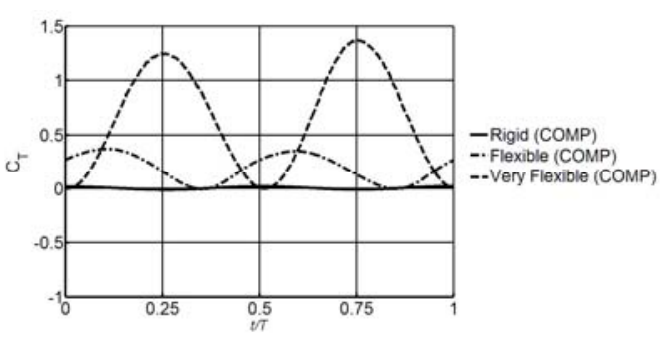

(b) $C_{T}$ generated by the flexible flat plate Figure V-2 Time histories of thrust coefficient contribution due to the teardrop and the flexible plate separately at $S t=0.17$ : (a) response of the teardrop; (b) response of the flexible plate. Extracted from [6].

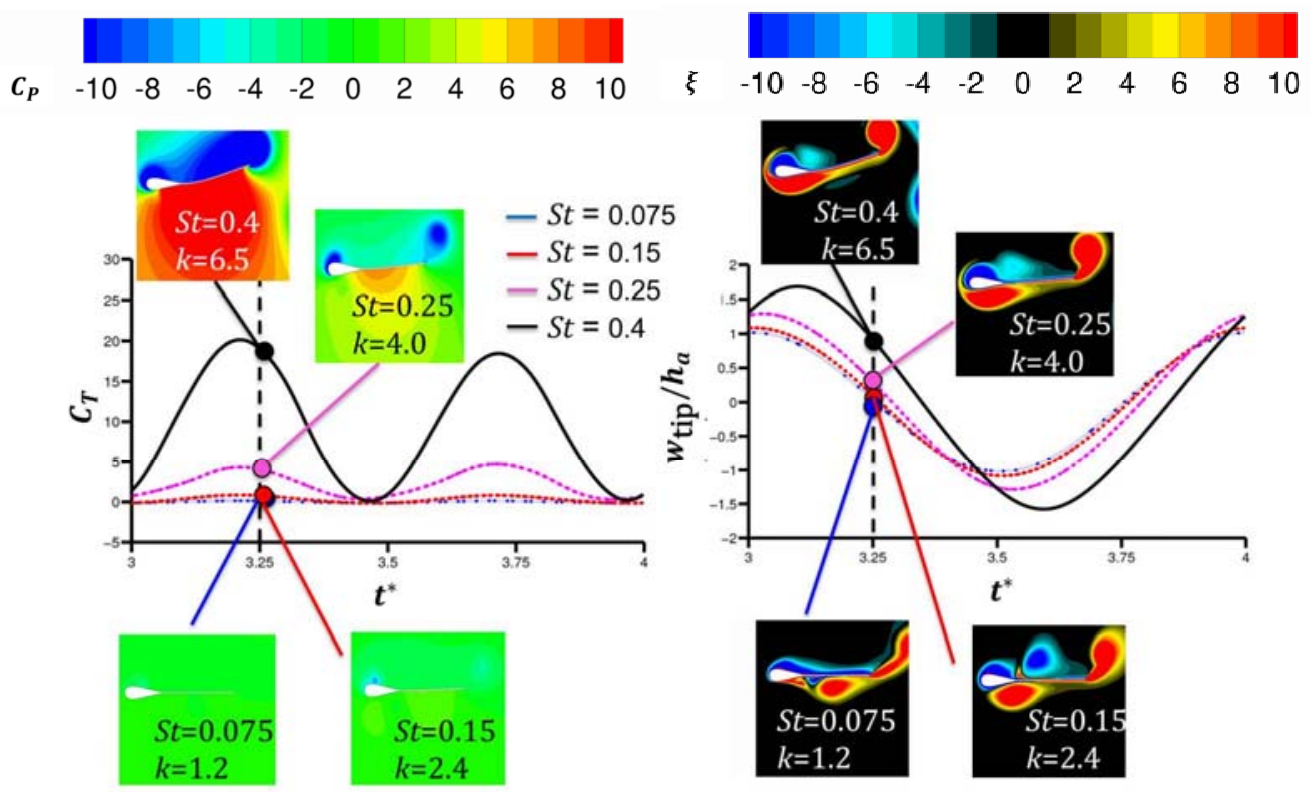

(a) Flexible $\left(h_{s}^{*}=1.41 \times 10^{-3}\right)$
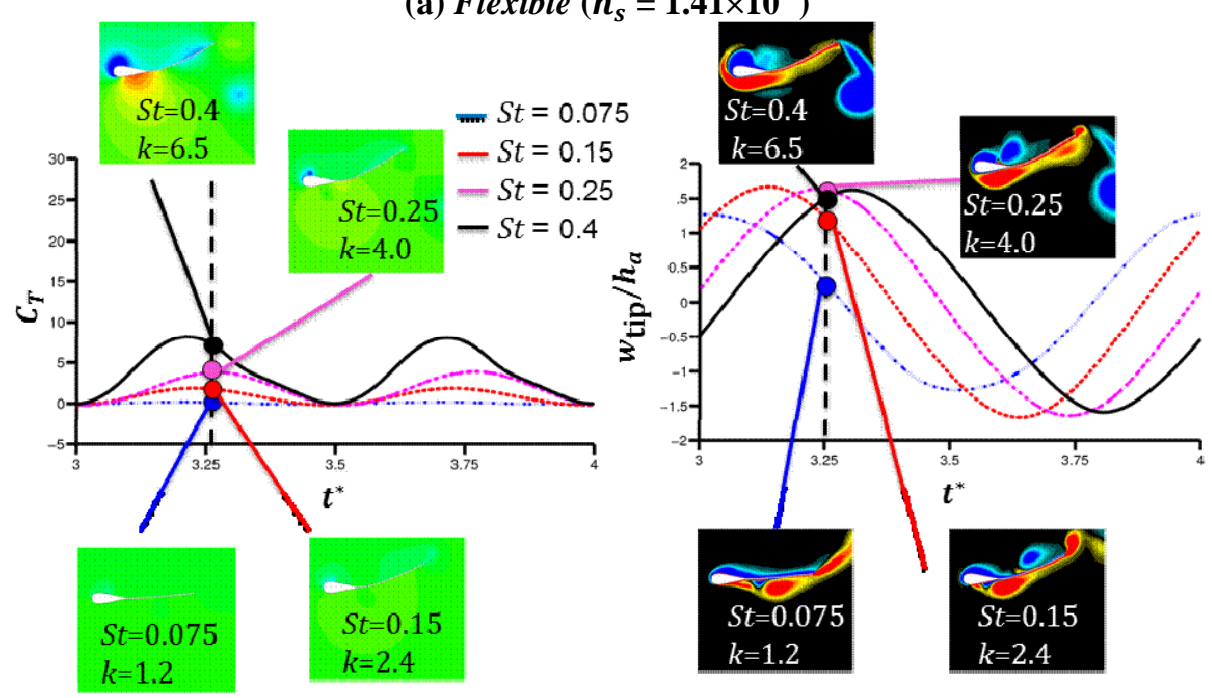

(b) Very Flexible $\left(h_{s}^{*}=0.56 \times 10^{-3}\right)$

Figure V-3 Time histories of thrust and wing tip displacement normalized by the plunge amplitude as function of non-dimensional time. Pressure coefficient and vorticity contours at $t^{*}=0.25$ for each Strouhal number are shown as well. $\left(\operatorname{Re}=9.0 \times 10^{3}\right.$ and $\left.\rho^{*}=7.8\right)$ 
The interplay between the motion frequency indicated with Strouhal number and the resulting thrust and wing tip displacement is further illustrated in Figure V-3. For the Flexible airfoil the resulting thrust generation increases with the increased motion frequency (Strouhal number and reduced frequency) and the maximum wing tip displacement also shows monotonic increase with the motion frequency. A striking observation is that the vorticity field looks similar for all Strouhal numbers shown, however the pressure contours and also the resulting thrust time histories differ in values. This could be related to the scaling proposed in Section IV that the force acting on a moving body is largely dominated by the motion of the airfoil and less with the vorticity in the flow field at high reduced frequencies. Similar trend is shown for the Very Flexible airfoil with the thickness ratio about 2.5 times smaller than for the Flexible airfoil cases. The thrust increases with higher $S t$ and $k$, however the maximum tip amplitude saturates for $S t=0.15,0.25$, and 0.4 . Instead of resulting in a larger tip amplitude motion, higher motion frequency leads to larger phase lag of the wing tip relative to the wing root.

Increasing motion frequency leads to higher acceleration of the wing, and hence greater force generation. However, eventually the fluid dynamics time scale and reponse become limiting factors, as it will be discussed in Sections V.1.D and V.2.

Figure V-4 shows the time-averaged thrust coefficient for a range of motion frequencies from the current numerical computation and the experimental measurements [64]. For the thickest flat plate $\left(h_{s}^{*}=4.23 \times 10^{-3}\right)$ the computed thrust compares well with the experimental measurements. At the higher motion frequencies, $S t=0.28$ and 0.3 , the computed thrust starts to deviate. Similar trend is observed for the other thicknesses: at $h_{s}^{*}=0.85 \times 10^{-3}$ the correlation between the numerical result and the experimental measurement is good until $S t=0.23$ and at $h_{s}^{*}=$ $0.56 \times 10^{-3}$ only at the lowest frequencies. Modeling uncertainties, such as laminar-to-turbulent transitions, nonlinearities in the structural modeling, or nonnegligible twist or spanwise bending in the experimental setup, which are not accounted for in the numerical computations may be attributed to the observed differences.

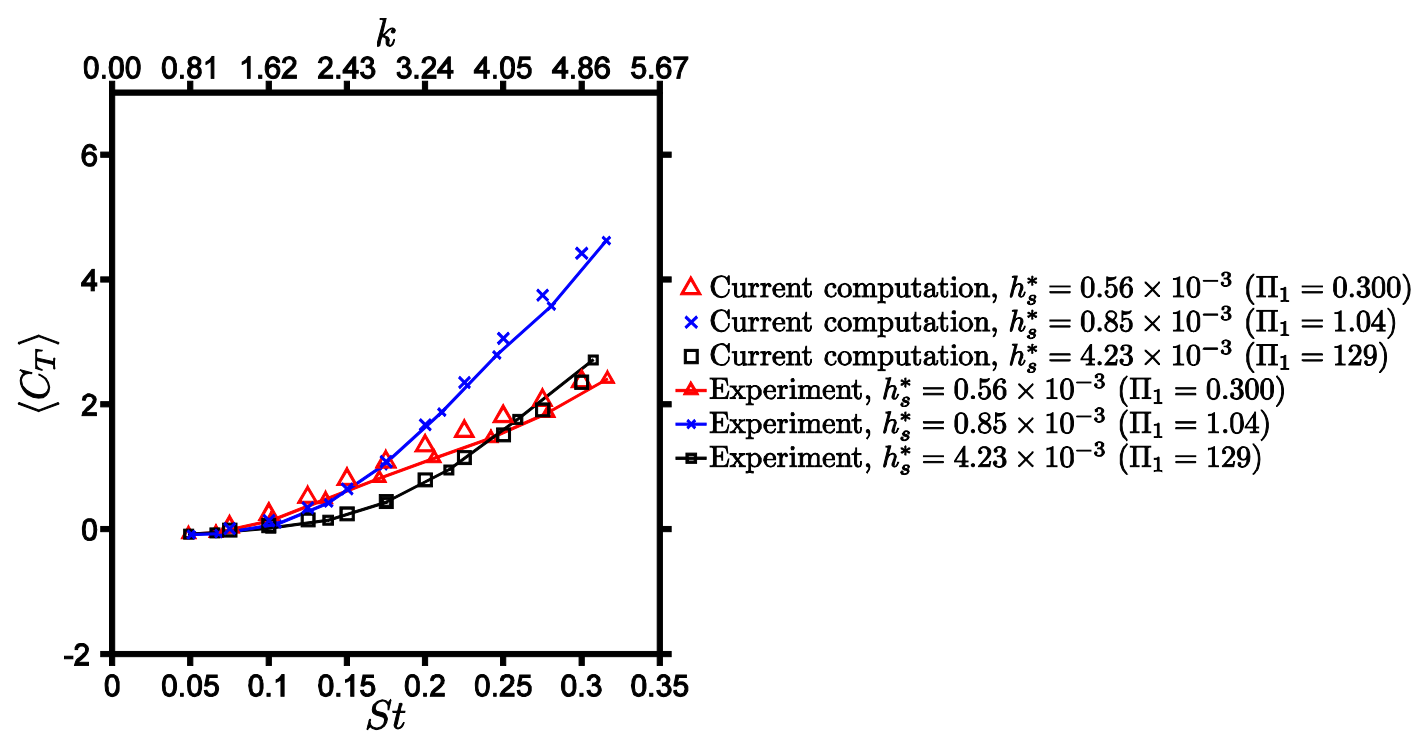

Figure V-4 Time-averaged thrust coefficient for a plunging chordwise flexible airfoil at $\operatorname{Re}=9.0 \times 10^{3}$ and $\rho^{*}$ =7.8 for different flat plate thickness and motion frequencies. The experimental data are extracted from [64]. 


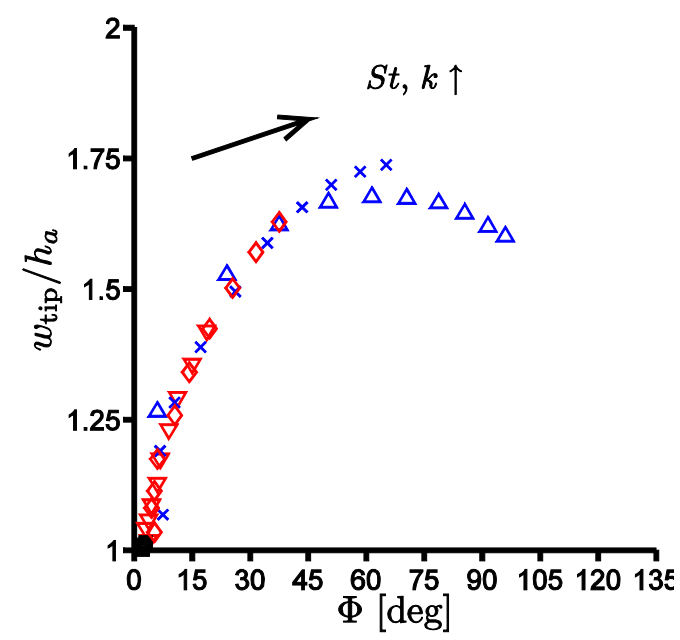

(a) Tip displacement

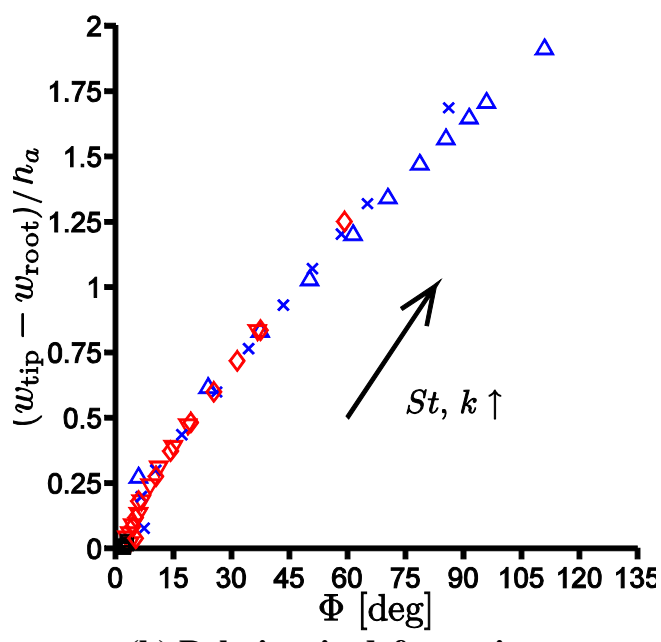

(b) Relative tip deformation

Figure V-5 Tip deformations of a plunging chordwise flexible airfoil at $R e=9.0 \times 10^{3}$ and $\rho^{*}=7.8$ for different flat plate thickness and motion frequencies. $\Delta h_{s}^{*}=0.56 \times 10^{-3} ; \times h_{s}^{*}=0.85 \times 10^{-3} ; \diamond h_{s}^{*}=1.13 \times 10^{-3}$; $\nabla h_{s}^{*}=1.41 \times 10^{-3} ; \square h_{s}^{*}=4.23 \times 10^{-3}$

The thrust for the thickest airfoil $\left(h_{s}^{*}=4.23 \times 10^{-3}\right)$ can be enhanced by increasing the motion frequency that results to higher $S t$ and $k$. Increased $S t$ leads to greater fluid dynamic force, but also greater added mass force. Furthermore, as the added mass force depends on the acceleration and the chord of the wing, see Eq. (17), higher $k$ will increase the added mass force further. Figure V-4 also shows that the thrust generation depends on the thickness of the wing: At $S t=0.125,\left\langle C_{T}\right\rangle$ for $h_{S}^{*}=0.56 \times 10^{-3}$ is the maximum; however, for higher Strouhal numbers the thrust generated by the thinnest airfoil is the lowest: at $S t=0.3, h_{s}^{*}=0.85 \times 10^{-3}$ generates the highest thrust, while the thinnest wing, $h_{s}^{*}=0.56 \times 10^{-3}$, deteriorate in thrust.

To characterize the structural response, the tip displacement normalized to the plunge amplitude, $w_{\text {tip }} / h_{a}$, is plotted in Figure V-5 as function of the phase lag relative to the leading edge for the thicknesses and frequencies considered. The phase lag $\Phi$ is calculated by determining the time instant at which the trailing edge displacement is a maximum. For the thickest airfoil, $h_{s}^{*}=4.23 \times 10^{-3}$, both the deformations and the phase lag are small. As we decrease the airfoil thickness, both $w_{\text {tip }} / h_{a}$ and $\Phi$ increase with increasing frequency, see Figure V-5. Eventually, $w_{\text {tip }} / h_{a}$ saturates when $\Phi$ approaches $90 \mathrm{deg}$ : when $\Phi>90$ deg the motion of the deformed trailing edge is out of phase with the imposed leading edge. Relative to the leading edge displacement, $\left(w_{\text {tip }}-w_{\text {root }}\right) / h_{a}$ shows that by decreasing the stiffness and increasing the motion frequency not only the tip deformation increases monotonically, but also the phase lag, so that the resulting wing tip displacement reduces in magnitude when the motion is out of phase (Figure V-5). In Section V.1.D a relationship between the mean thrust and the structural response will be established.

\section{V.1.B Purely Plunging Spanwise Flexible Wing in Forward Flight in Water}

The effects of spanwise flexibility on thrust generation of a three-dimensional rectangular wing oscillating in pure plunge in forward flight have been investigated with water tunnel experiments [65] and numerical simulations $[52,6,66]$. The wing models of $R=0.3 \mathrm{~m}$ semi-span and $c_{\mathrm{m}}=0.1 \mathrm{~m}$ chord length with several spanwise flexibilities were considered. More detailed information of experimental case setup can be obtained in Ref. [65]. In this study, two combinations of density ratio and effective stiffnesses and several motion frequencies at $R e=3.0 \times 10^{4}$ are considered to compare the results with available experimental and computational results, to highlight the thrust enhancement mechanism associated with spanwise flexible plunging wings in forward flight. The grid and time step sensitivity studies are shown in Appendix A.2.

The vertical displacements of the wing tip from the computations and the experiments for the Flexible and Very Flexible wings are shown in Figure V-6. The displacement is normalized with respect to the amplitude of prescribed wing root movement. For the Flexible wing, in comparison to the tip response presented in previous studies (experiment: [65]; implicit Large Eddy Simulation (iLES) computation [66]), the tip response of current computation shows good correlation. For the Very Flexible wing, however, the tip response of the current computation exhibits slight larger amplitude and phase advance compared to the measurements [65]. 


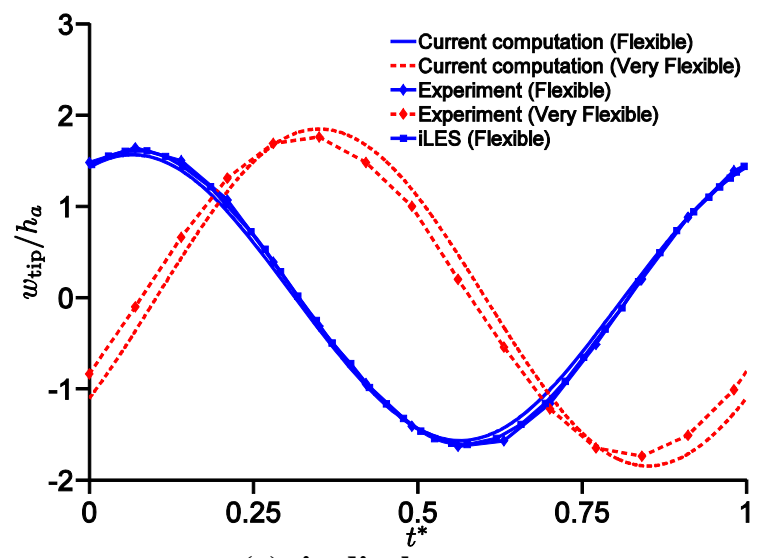

(a) tip displacement

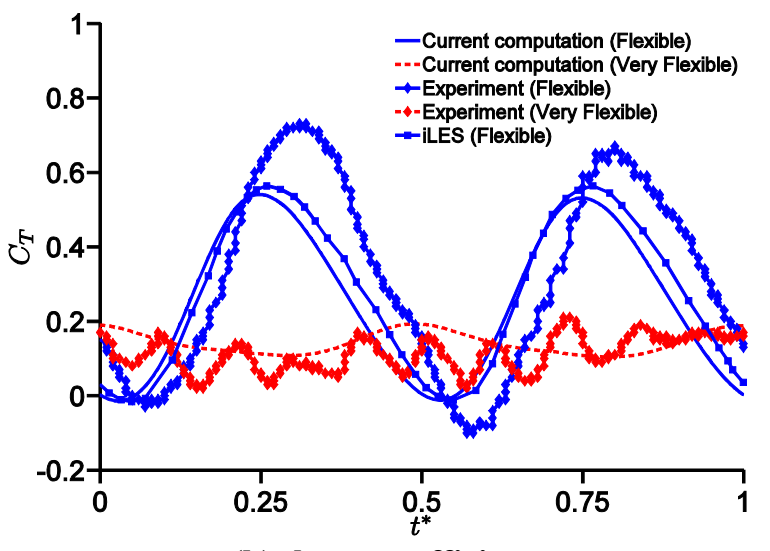

(b) thrust coefficient

Figure V-6 Time history of (a) tip displacements and (b) thrust coefficient of a plunging spanwise flexible wing at $R e=3.0 \times 10^{4}$ for different wing stiffness, wing density, and motion frequencies. The experimental data are extracted from Ref. [65] and the implicit LES from [66].
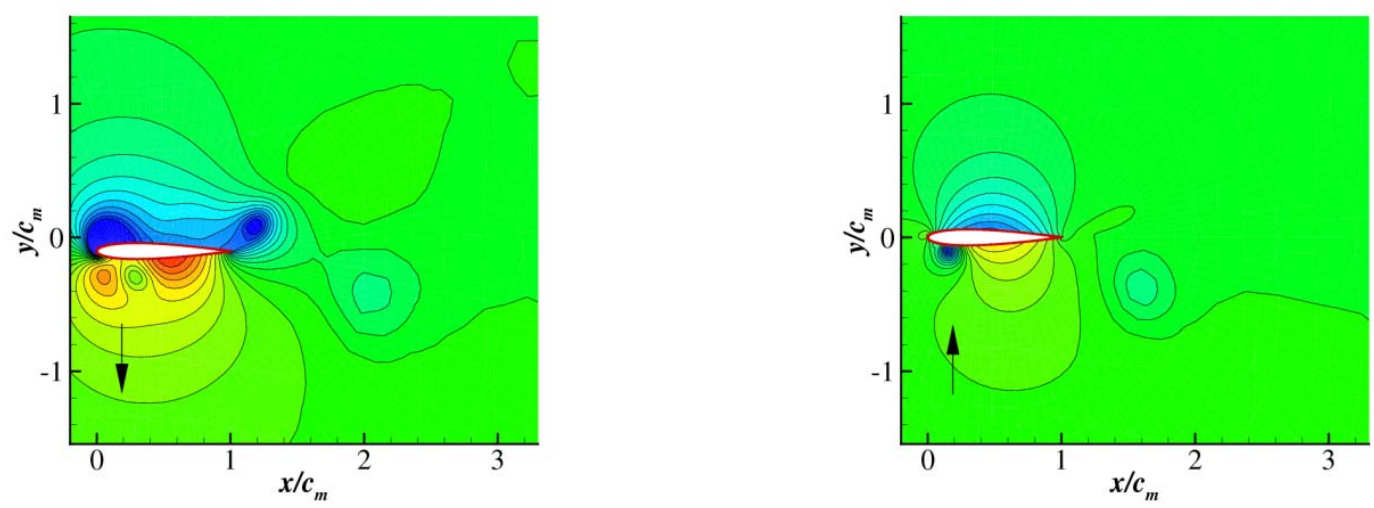

(a) pressure coefficient contour levels: 2; range: -2.5 to 2.5

$$
h_{s}^{*}=0.01, S t=0.1, k=1.82, t^{*}=2.25
$$

(left) $\Pi_{1}=212, \rho^{*}=7.8$; (right) $\Pi_{1}=38, \rho^{*}=2.7$
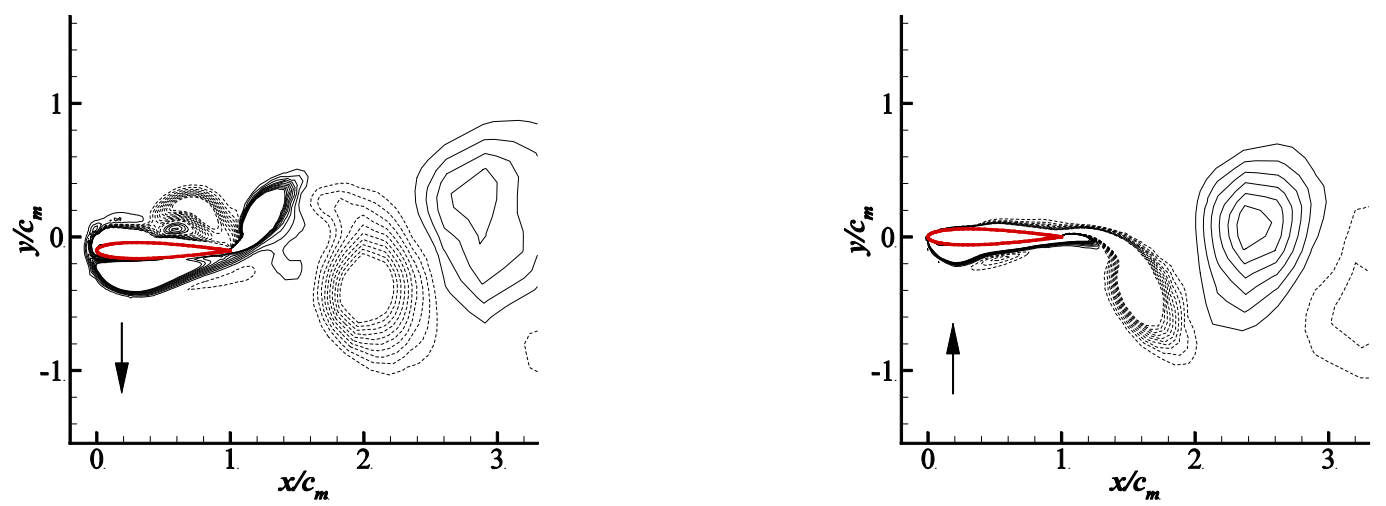

(b) vorticity contour levels: 20 ; range: -3 to 3

$$
h_{s}^{*}=0.01, S t=0.1, k=1.82, t^{*}=2.25
$$

(left) $\Pi_{1}=212, \rho^{*}=7.8$; (right) $\Pi_{1}=38, \rho^{*}=2.7$

Figure V-7 Pressure coefficient $\left(\boldsymbol{p}-\boldsymbol{p}_{\infty}\right) /\left(\frac{1}{2} \rho_{f} U_{\text {ref }}^{2}\right)$ and vorticity contours at $75 \%$ span location for Flexible and Very Flexible wing configurations. The arrow indicates the direction of the airfoil motion. 
Time histories of thrust coefficient for the Flexible and Very Flexible wings are shown in Figure V-6. For the Flexible wing the thrust in the current computation is underpredicted and has some phase advance compared to the measurements [65]. However, the agreement in terms of the magnitude and the timing of thrust peaks compares well with the thrust prediction using the iLES [66] coupled with a geometrically nonlinear beam solver. Furthermore, the measured thrust is assymetric in the donwstroke and upstroke while in both computations the thrust has symmetric behaviour. For the Very Flexible wing, the computed thrust history is in a reasonable agreement with the experimental measurements in terms of the amplitude and the trend of thrust. It is worth to point out that the measurements include higher frequency components, while the waveforms of the computed thrust are smooth for all cases. As shown in Figure V-6, there is no evidence of high frequency behaviour in the tip response. The experimental flow field measurements [65] also did not indicate flow features that may be attributed to these high frequency contents. Therefore this difference may have risen from uncertainties in the experimental setup or in the computational modelling. To further quantify these uncertainties comparisons of instantaneous three-dimensional wing shapes and flowfields near the leading-edge are recommended between the experiments and computations. Furthermore, more detailed documentation of the torsion and the natural frequencies measurements of the wing would be helpful for computational modeling.

Vorticity and pressure contours for the Flexible and the Very Flexible wing configurations at the mid-span section at time instant $t^{*}=0.25$, when the wing is at the center of downstroke, are shown in Figure V-7. The dominance of leading edge suction in the Flexible case and the reduction of it in the Very Flexible case are visible in that figure. The phase lag between the prescribed motion and the deformation of the wing is could be used to explain the thrust generation in flexible flapping wings [6]. For the Very Flexible case the cross sectional motion is in the opposite direction of the imposed kinematics at the wing root. The phase lag at the wing tip with respect to the prescribed motion for the Flexible and Very Flexible cases are, -26 deg, and -126 deg, respectively. As a result of the substantial phase lag in the Very Flexible case, the wing tip and root move in opposite directions during most of the stroke resulting in lower effective angles of attack and consequently lesser aerodynamic force generation, see also the direction of the arrow in the contour plots that denotes the direction of the wing movement.

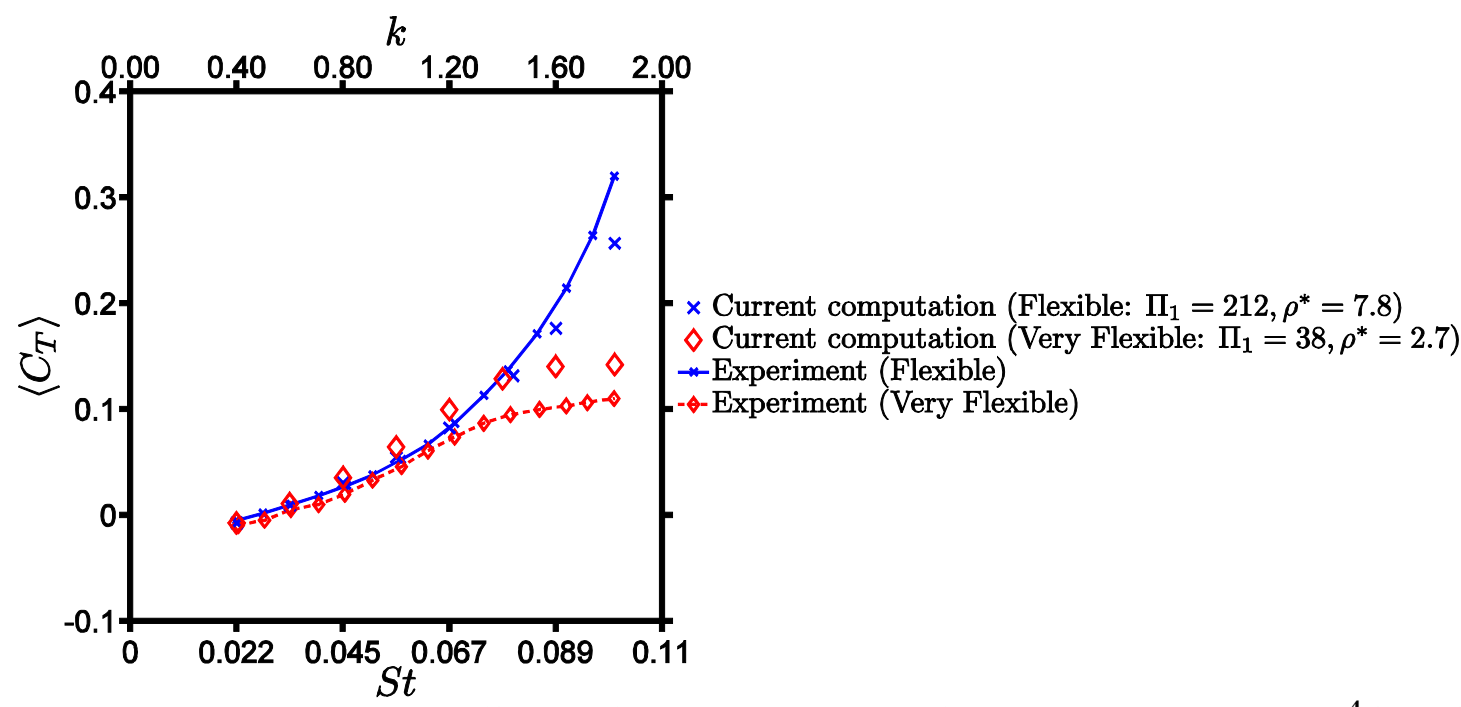

Figure V-8 Time-averaged thrust coefficient of a plunging spanwise flexible wing at $R e=3.0 \times 10^{4}$ for different wing stiffness, wing density, and motion frequencies. The experimental data are extracted from Ref. [65].

Figure V-8 shows the time-averaged thrust coefficients for the two different materials for various frequencies of $k=0.4,0.6,0.8,1.0,1.2,1.4,1.6$, and 1.82. At higher motion frequencies the time-averaged thrust of the Flexible wing is underpredicted by the current computational results, whereas those of the Very Flexible wing is being overpredicted, which again may be ascribed to the uncertainties in the computational modeling or experimental setup. However, the qualitative trend of the thrust response to the variation of the motion frequency is well-captured. When the plunging motion is slow, i.e. $k<1.2$ the thrust generation is similar for both materials. For higher motion frequecies the Flexible wing benefits more from the flexibility than that of the Very Flexible wing: the thrust saturates for the Very Flexible wing with increasing St Furthermore, similar trends are observed: increasing motion frequency enhanced thrust and decreasing the effective stiffness doesn't necessarily lead to higher thrust. 


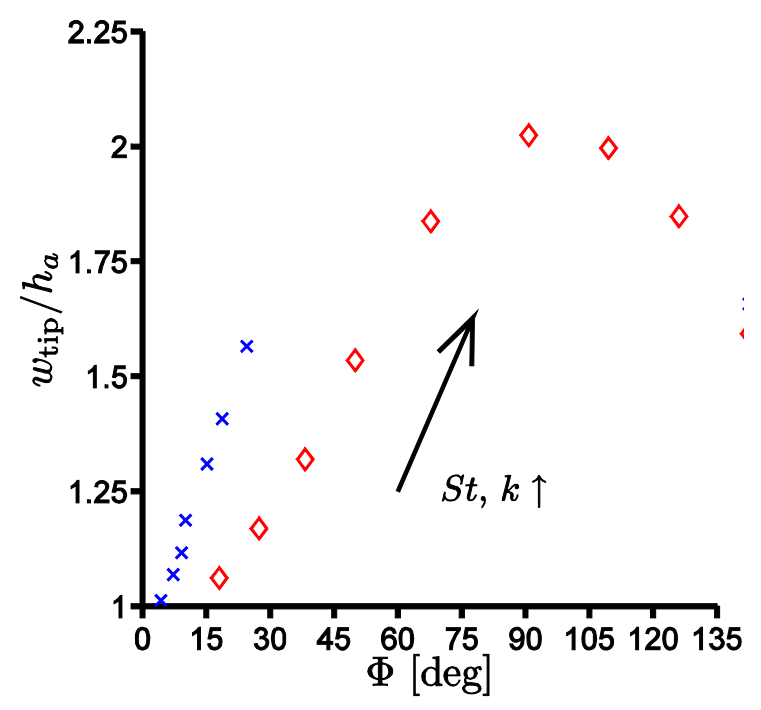

(a) Tip displacement

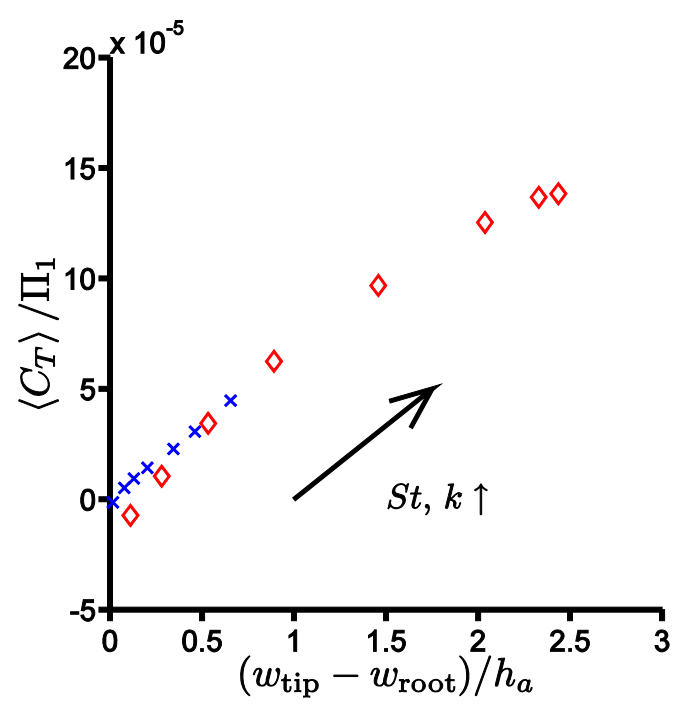

(b) Relative tip deformation

Figure V-9 Tip deformations of a plunging spanwise flexible wing at $R e=9.0 \times 10^{3}$ for different wing stiffness, wing density, and motion frequencies. $\times \Pi_{1}=121, \rho^{*}=7.8 ; \diamond \Pi_{1}=38, \rho^{*}=2.7$

The structural response is depicted in Figure V-9. For the Flexible wing, the phase lag between the prescribed motion and the tip response for $k>1.4$ is from $17.6 \mathrm{deg}$ to $23.8 \mathrm{deg}$, whereas for the Very Flexible wing, $\Phi$ varies from 108.8 deg to $125.9 \mathrm{deg}$, see Figure V-8. The wing tip of the Very Flexible wing moves in opposite direction as the root for the most of the stroke for higher motion frequencies, while for the Flexible wing, the wing root and the tip are in phase. This is confirmed in Figure V-9 where all Flexible wing cases show a phase lag of the wing tip relative to the wing root, $\Phi$, less than $90 \mathrm{deg}$, while for $k=1.6$ and $k=1.82 \Phi>90 \mathrm{deg}$ for the Very Flexible wing. Again, the correlations of the dynamics from the root to tip play a key role for the tip displacement as shown in Figure V-9, where the relative tip displacement is shown to be monotonic to $\Phi$. Moreover, the relationship between the time-averaged thrust and the relative tip displacement is discussed in Section V.1.D.

\section{V.1.C Hovering Isotropic Zimmerman Wing in Air}

The previous two cases were proposed by Heathcote and Gursul [64] and Heathcote, Wang, and Gursul [65] to assess the effects of chordwise and spanwise flexibilities, respectively, on the thrust generation of plunging wing in forward flight at low density ratio. Motivated by the experimental studies on the three-dimensional Zimmerman wing planform [31], a three-dimensional hovering flapping isotropic wing in air is considered. The wing is a flat plate wing of $c_{\mathrm{m}}=0.0196 \mathrm{~m}$ and $A R=3.825$ with a thickness ratio of $h_{s}^{*}=2.0 \times 10^{-2}$ having a Zimmerman planform, see Figure V-10, hovering in air at $R e=1.5 \times 10^{3}$. A sinusoidal flapping motion is introduced at the rigid triangle at the leading edge at the wing root following, Eq. (3) with $S t=0.25$ and $k=0.56$. The flapping axis is parallel to the wing root. Note that in the axes definition by Wu et al. [6] the wing flaps up-and-down to generate thrust due to wing flexibility, however in the current study the flapping wing axis has been rotated so that the flapping axis is parallel to the lift direction, such that the any flexibility in the wing leads to lift generation. The triangular rigid region near the root at the leading edge undergoes prescribed motion and is constrained in all degrees of freedom in the structural solver, since the flapping mechanism in the experiment [67] is actuated at this region on the wing. The grid and time step sensitivity studies are shown in Appendix A.3. 


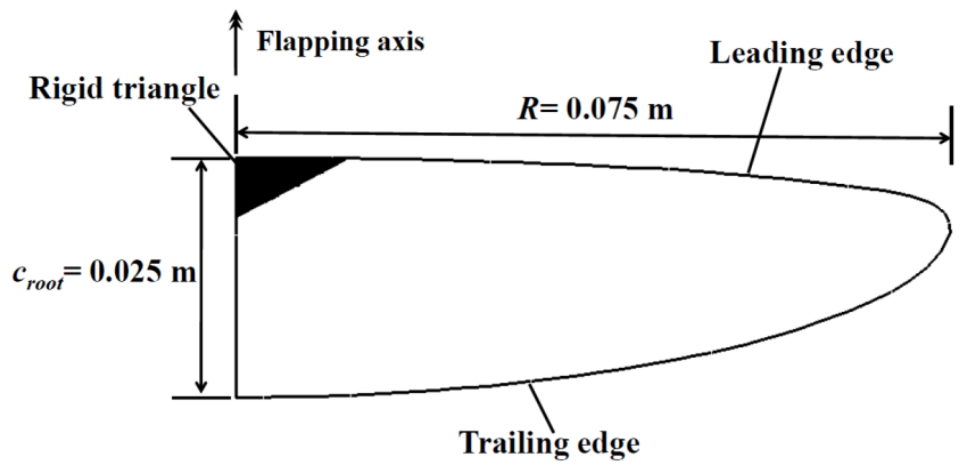

Figure V-10 Geometry of the Zimmerman planform.

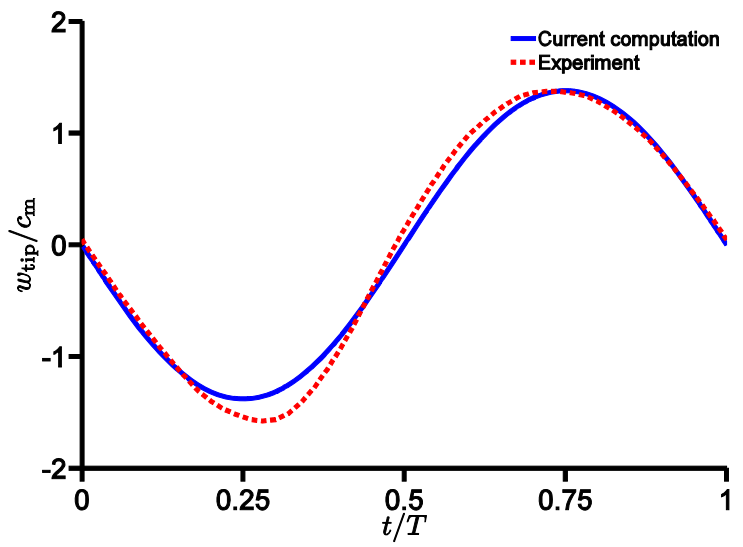

Figure V-11 Time histories of horizontal displacement at the wing tip of a flapping isotropic Zimmerman wing hovering at $R e=1.5 \times 10^{3}, k=0.56$, and $S t=0.25$, made of aluminum.

To validate the numerical computation the Zimmerman wing made of aluminum is compared to the available experimental data [67] in terms of the wing tip displacement in horizontal direction in Figure V-11. The predicted tip response shows reasonable agreement with the measured displacement and captures the main qualitative trends of the response of wing tip. The current computation exhibits a more symmetric response between the downstroke and the upstroke in comparison to the experimental measurements. The reason for the asymmetry in the measurements might be due to uncertainties in the experimental setup related to the driving system of the flapping device, or cycle-to-cycle variations in the measurements.

To assess the effects of different wing properties: the effective stiffness $\Pi_{1}$ and the density ratio $\rho^{*}$, on the resulting lift and wing deformations, surrogate models are constructed to qualitatively explore their implications. Surrogate models offer methods to efficiently organize the data measured as objective functions and give global and reliable qualitative trend as function of design variables [58]. The range for these variables in the design space is chosen to cover wide range of applications as shown in Table V-1. To effectively assess the order of magnitude of the design variables a logarithmic scaled design space will be populated.

Table V-1. Range of the design variables $\Pi_{1}$ and $\rho^{*}$ with representative examples.

\begin{tabular}{ccc}
\hline \hline Parameter & Minimum & Maximum \\
\hline$\Pi_{1}$ & $10^{2}$ (High density polyethylene in air) & $10^{5}$ (steel, aluminum in air) \\
$\rho^{*}$ & $10^{1}$ (water to steel, aluminum) & $10^{4}$ (air to steel, aluminum) \\
\hline \hline
\end{tabular}

The objective functions are (a) the lift coefficient averaged over one motion cycle between the second and the third cycle, i.e. $m=2.5$ in Eq. (6), (b) the twist angle $\theta$ given as

$$
\theta=\max \left\{\operatorname{acos}\left(\boldsymbol{c}_{3} \cdot \boldsymbol{e}_{1}\right)\right\} .
$$


where $c_{3}$ is the unit vector in the direction from the leading edge to the trailing edge at the section 3 of the wing (see Section A.3) and $\boldsymbol{e}_{1}$ is the unit vector in the lift direction. So $\theta$ gives the degree of the lift favorable projectional area of the wing due to the deformation, and (c) the bending angle $\psi$ that is defined as

$$
\psi=\max \left\{\operatorname{atan}\left(\frac{w}{R}\right)-\phi\right\} .
$$

to measure the wing deformation in spanwise direction $x$ as the maximum tip displacement angle relative to the imposed flap angle $\phi$. For simplicity lift will be referred as the time-averaged lift coefficient from now on.

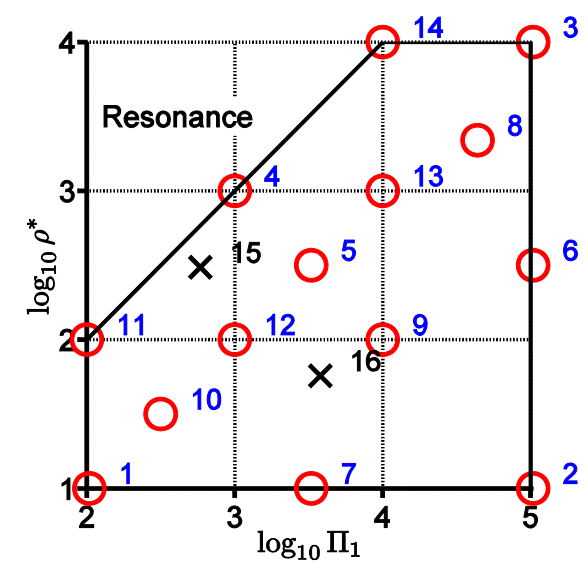

Figure V-12 Design of experiment in logarithmic scale for the design variables $\Pi_{1}$ and $\rho^{*}$. The training points are indicated by cicles and the training points by crosses.

The design of experiments use a face centered cubic design (FCCD) and then the remainder of the design space is filled evenly in the design space with the cases 8 and 10 together with the testing points are generated by the latin hypercube algorithm. In total 14 training points are selected. A tabulation of the training points are found in Table B-1 in the Appedix B. The design space with logarithmic bias towards the softer $\Pi_{1}$ and lighter $\rho^{*}$ structures are shown in Figure V-12. The region where $\log _{10} \rho^{*}>\log _{10} \Pi_{1}+2$ is out of the scope of the current study as this region showed largely unstable behaviour of the wing motion because the imposed frequency of $10 \mathrm{~Hz}$ is close to the natural frequencies, see 0 .

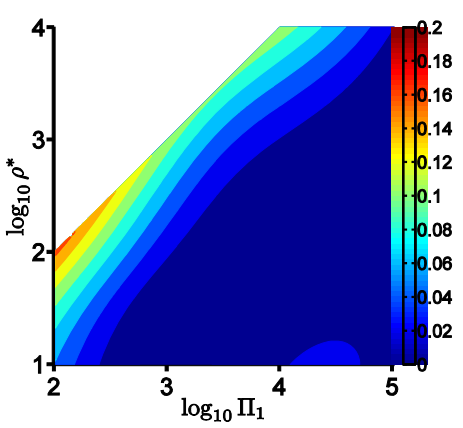

(a) $\left\langle C_{L}\right\rangle$

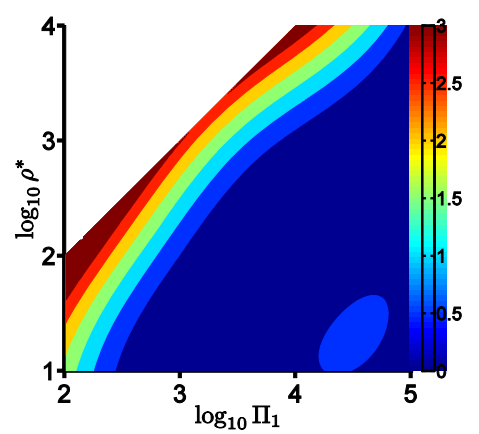

(a) $\theta$

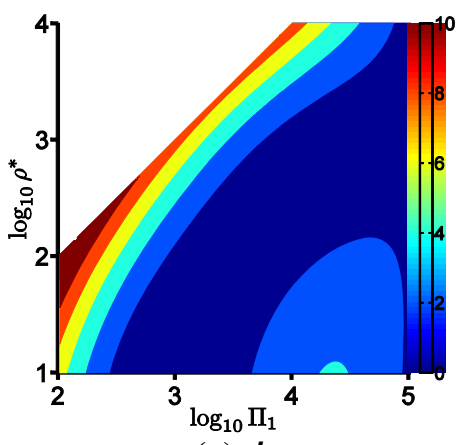

(a) $\psi$

Figure V-13 Surrogate model responses for (a) lift, (b) twist, and (c) bending angles for a flapping isotropic Zimmerman wing, hovering at $R e=1.5 \times 10^{3}$ and $k=0.56$.

The resulting surrogate models are shown in Figure V-13 for the lift, twist, and bending angle. Notice that the time-averaged lift for the rigid wing would be zero due to the symmetry in the hovering kinematics without pitching motion. The lift, twist, and bending are at the maximum at the case 4 and these three objective functions have qualitatively similar trend in the design space suggesting that there exists a correlation between the resulting timeaveraged lift force and the maximum deformations. Furthermore, it is not only the effective stiffness $\Pi_{1}$, or the density ratio $\rho^{*}$, but the balance between these two parameters that determine the resulting deformation and the lift 
generation. The region of increased objective functions between $\log _{10} \Pi_{1}=4$ and 5 and $\log _{10} \rho^{*}=1$ to 2 is caused by the error in surrogate model due to high gradient near the resonance region, yet wide region of almost zero values at more stiffer and lighter portion of the design space.

As the sinusoidal rigid-body motion is imposed at the triangular rigid part near the wing root (see Figure V-10), the wing inertia and the resulting aerodynamic load are balanced out by the elastic force. Since the wing is made of isotropic material the structure will respond in both spanwise bending as well as twisting.

For the chordwise flexible airfoil in Section V.1.A and spanwise flexible wing in Section V.1.B the thrust generation in forward flight was shown to be dominantly dependent on the resulting tip motion relative to the imposed kinematics at the wing root. For the flapping Zimmerman wing in hover in air, maximum horizontal tip displacement $w$ normalized by the prescribed amplitude $h_{a}=R \sin \phi_{a}$ is plotted against the phase lag with respect to the top of the stroke of the rigid body motion in Figure V-14. The higher tip amplitude corresponds with larger phase lag compared to the imposed kinematics, while the tip motion is in phase. The cases with the lowest $\Pi_{1} / \rho^{*}$ ratio have larger deformation consistent with the surrogate model responses shown in Figure V-13.

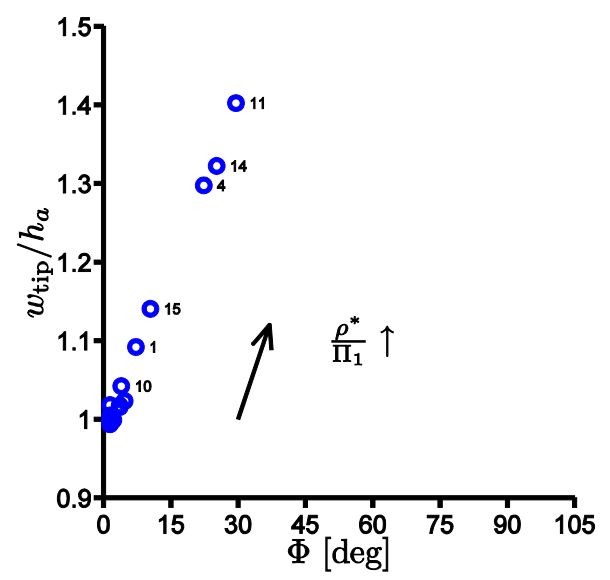

Figure V-14 Tip deformations of a flapping isotropic Zimmerman wing hovering in air at $R e=1.5 \times 10^{3}$ and $k$ $=0.56$ for different $\rho^{*}$ and $\Pi_{1}$. The numbers shown next to the markers indicate their case numbers.

\section{V.1.D Scaling Parameterfor the Force Generation}

From the results presented in Section V.1.A, V.1.B, and V.1.C for the three different cases we have observed the followings: i) time-averaged force increased with increasing motion frequency; ii) the effects of change in structural properties, such as the thickness ratio, Young's modulus, or wing density (mass), led to non-monotonic response in the force generation; iii) for the hovering isotropic Zimmerman wing the ratio between the density ratio and the effective stiffness was monotonic with the time-averaged lift generation. To explain the observed trends we will mainly analyze the physics based on Eq. (2) with simplifying approximations for the fluid dynamic force, $f_{f}^{*}$ (see also Section IV.1), based on scaling arguments. The flow field and the structural displacement field should simultaneously satisfy Eq. (1) and Eq. (2) and among these two Eq. (2) will be considered, which has the advantage that this equation is linear except for the fluid dynamic force term as opposed to the Navier-Stokes equation which is nonlinear in the convection term. Subsequently, we will establish a relation between the time-averaged force and the maximum relative tip displacement by considering the energy balance.

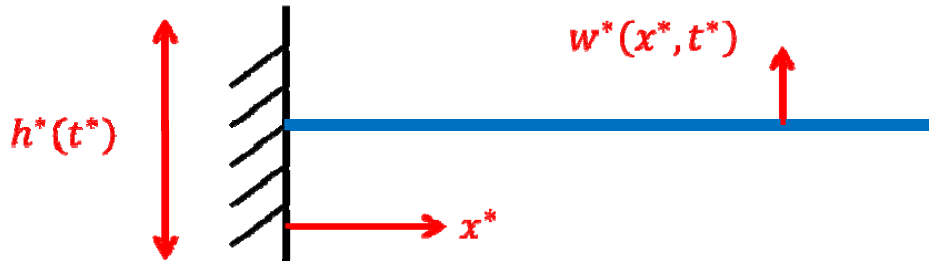

Figure V-15 Schematic of the wing approximated as beam and the definitions of the wing deformation $w^{*}$, prescribed motion at the root $h^{*}$, and the spatial coordinate $x^{*}$. 
To capture the essence of the mechanism involved in the force enhancement due to the flexibility, the interplay between the imposed kinematics, the structural response of the wing, and the fluid force acting on the wing are analyzed. The derivation leading to the relation between the time-averaged force acting perpendicular to the wing motion, $\left\langle C_{F}\right\rangle$ and the maximum relative tip deformation $v_{\max }$, where $v^{*}\left(x^{*}, t^{*}\right)=w^{*}\left(x^{*}, t^{*}\right)-h\left(t^{*}\right)$ is the displacement of the wing is relative to the imposed kinematics motion, see Figure V-15, is lengthy and many of the steps are similar to those discussed in classical textbooks (e.g. [68]), however to account for the approximations involved full derivation is presented. Such treatment involving simplifying approximation is helpful, enabling the analysis, but mainly serves to elucidate the scaling analysis, not meant to offer complete solutions. Consider Eq. (2) in one-dimension in space with $0 \leq x^{*} \leq 1$ and time $t^{*} \geq 0$ for the vertical displacement $w^{*}$ with the wing approximated as a linear beam, i.e.

$$
\Pi_{0}\left(\frac{L}{c_{\mathrm{m}}}\right) \frac{\partial^{2} w^{*}}{\partial t^{* 2}}+\Pi_{1}\left(\frac{c_{\mathrm{m}}}{L}\right)^{3} \frac{\partial^{4} w^{*}}{\partial x^{* 4}}=f_{f}^{*}
$$

where $f_{f}^{*}$ is the fluid force on the wing. A plunge motion Eq. (4) is imposed at the leading edge at $x^{*}=0$. At the trailing edge at $x^{*}=1$ is considered as a free end, i.e. with the boundary conditions

$$
\begin{gathered}
w^{*}\left(0, t^{*}\right)=h\left(t^{*}\right)=S t \frac{\pi}{k} \cos \left(2 \pi t^{*}\right), \\
\frac{\partial w^{*}\left(0, t^{*}\right)}{\partial x^{*}}=\frac{\partial^{2} w^{*}\left(1, t^{*}\right)}{\partial x^{*}}=\frac{\partial^{3} w^{*}\left(1, t^{*}\right)}{\partial x^{*}}=0
\end{gathered}
$$

and the initial conditions

$$
w^{*}\left(x^{*}, 0\right)=S t \frac{\pi}{k}, \quad \frac{\partial w^{*}\left(x^{*}, 0\right)}{\partial t^{*}}=0,
$$

where the factors involving $L / c_{\mathrm{m}}$ become unity for the chordwise flexible airfoil case. For the spanwise flexible wing and the isotropic Zimmerman wing cases which are discussed in Section V.1.B and Section V.1.C, respectively, $\Pi_{0}$ and $\Pi_{1}$ need to be corrected as $L / c_{\mathrm{m}}=A R$. Following the procedure described in Mindlin and Goodman [69], a PDE with homogeneous boundary conditions can be found by superimposing the plunge motion on the displacement $v\left(x^{*}, t^{*}\right)=w\left(x^{*}, t^{*}\right)-h\left(t^{*}\right)$, which gives

$$
\Pi_{0} \frac{\partial^{2} v^{*}}{\partial t^{* 2}}+\Pi_{1} \frac{\partial^{4} v^{*}}{\partial x^{* 4}}=f_{f}^{*}-\Pi_{0} \frac{d^{2} h\left(t^{*}\right)}{d t^{* 2}}
$$

for the PDE and

$$
v^{*}\left(0, t^{*}\right)=\frac{\partial v^{*}\left(0, t^{*}\right)}{\partial x^{*}}=\frac{\partial^{2} v^{*}\left(1, t^{*}\right)}{\partial x^{* 2}}=\frac{\partial^{3} v^{*}\left(1, t^{*}\right)}{\partial x^{* 3}}=0,
$$

and the initial conditions

$$
v^{*}\left(x^{*}, 0\right)=\frac{\partial v^{*}\left(x^{*}, 0\right)}{\partial t^{*}}=0 .
$$

The consequence of having a sinusoidal displacement at the root is that the vibrational response of the wing is equivalent to a sinusoidal excitation force, which is the inertial force. The dynamic motion given by Eq. (24) is coupled to the fluid motion via the fluid force term $f_{f}^{*}$ which cannot be solved in a closed form due to its nonlinearities. For high density ratio FSI systems, Daniel and Combes [70] and Combes and Daniel [71] have shown that the inertial force arising from the wing motion is larger than the fluid dynamic forces. In this study to cover wider range of density ratios the fluid dynamic forces are included by considering the added mass effects. The motivation stems from the scaling discussed in Section IV.1 that for high $k$ the added mass terms due to an accelerating body (see also [72]) contribute more on the wing than the fluid dynamic forces from the hydrodynamic impulse, see Table V-2 for a summary of the non-dimensional numbers considered in this study. Hence, the wing 
dynamics is modeled with external forces depending on the imposed wing acceleration as

$$
f_{f}^{*}\left(t^{*}\right)=2 \pi^{2} S t k \cos \left(2 \pi t^{*}\right),
$$

hence the external force on the structural dynamics does not have spatial distribution explicitly accounted for and the external force is being simplified in temporal form. Combined with the inertial force the total external force $g\left(t^{*}\right)$ becomes

$$
g(t)=f_{f}^{*}\left(t^{*}\right)-\Pi_{0} \frac{d^{2} h\left(t^{*}\right)}{d t^{* 2}}=2 \pi^{2}\left(1+\frac{4}{\pi} \rho^{*} h_{s}^{*}\right) S t k \cos \left(2 \pi t^{*}\right) .
$$

Table V-2 Range of the non-dimensional parameters considered in this study. The aspect ratio of the twodimensional chordwise flexible airfoil is set to 1 for the reasons discussed in Section II. The representative Section numbers are shown in the parentheses.

\begin{tabular}{cccc}
\hline \hline Case & Chordwise (Section V.1.A) & Spanwise (Section V.1.B) & Isotropic (Section V.1.C) \\
\hline$R e\left[10^{3}\right]$ & 9.0 & 30 & 1.5 \\
$A R$ & 1 & 3.0 & 3.825 \\
$h_{S}^{*}\left[10^{-3}\right]$ & $0.56 \sim 4.23$ & 10 & 20 \\
$\rho^{*}$ & 7.8 & $2.7 \sim 7.8$ & $10^{1} \sim 10^{4}$ \\
$\Pi_{1} / A R^{3}$ & $0.3 \sim 129$ & $7.9,1.4$ & $1.794 \sim 1794$ \\
$k$ & $1.2 \sim 6.5$ & $0.4 \sim 1.82$ & 0.56 \\
$S t\left[10^{-2}\right]$ & $7.5 \sim 40$ & $2.0 \sim 10$ & 25 \\
\hline
\end{tabular}

Equation (24) can be solved using the method of separation, i.e. $v(x, t)=X(x) T(t)$, resulting in

$$
\begin{gathered}
\frac{d^{4} X}{d x^{* 4}}-k_{n}^{4} X=0, \\
\frac{d^{2} T}{d t^{* 2}}+\omega_{n}^{2} T=Q_{n} g\left(t^{*}\right),
\end{gathered}
$$

where $Q_{n}\left(t^{*}\right)$ is a Fourier coefficient of a unit function in the spatial modes $X_{n}$ satisfying,

$$
\begin{aligned}
& \sum_{n=1}^{\infty} Q_{n} X_{n}=1 \\
& Q_{n}=\frac{\int_{0}^{1} X_{n} d x^{*}}{\int_{0}^{1} X_{n}^{2} d x^{*}}
\end{aligned}
$$

where we have normalized $X_{n}$, i.e.

$$
\int_{0}^{1} X_{n}^{2} d x^{*}=1
$$

The equation and the boundary conditions for $X(x)$ is the same as for a free vibrating cantilever beam, of which the solution is given numerous textbooks, e.g. [68]. The natural frequency is given by

$$
\omega_{n}^{2}=\left(\frac{k_{n} 1}{1}\right)^{4} \frac{\Pi_{1}}{\Pi_{0}}=\left(\frac{2 \pi f_{n}}{f}\right)^{2}
$$


where $f_{n}$ is the natural frequency of the beam, i.e.,

$$
\left(2 \pi f_{n}\right)^{2}=\left(\frac{k_{n} 1}{1}\right)^{4} \frac{E I}{\rho_{s} A_{c}}
$$

where $I$ is the moment of inertia, $A_{c}$ is the cross sectional area of the wing represented as a beam, and $k_{n}$ is the eigenvalue belonging to the spatial mode $X_{n}$ that satisfies the transcendental equation

$$
\cos \left(k_{n} L\right) \cosh \left(k_{n} L\right)=-1,
$$

and can be approximated by the formula [68]

$$
k_{n} L \approx\left(n-\frac{1}{2}\right) \pi
$$

where $k_{1} L \approx 1.875$ and finally the spatial modes are given by

$$
X\left(x^{*}\right)=-\frac{1}{2}\left[\left\{\cos \left(k_{n} x^{*}\right)-\cosh \left(k_{n} x^{*}\right)\right\}-\frac{\cos \left(k_{n}\right)+\cosh \left(k_{n}\right)}{\sin \left(k_{n}\right)+\sinh \left(k_{n}\right)}\left\{\sin \left(k_{n} x^{*}\right)-\sinh \left(k_{n} x^{*}\right)\right\}\right] .
$$

The initial position of the beam is consistent with the imposed boundary condition. The solution for the temporal equation in $T\left(t^{*}\right)$ is

$$
T_{n}\left(t^{*}\right)=\frac{2 \pi\left(1+\frac{4}{\pi} \rho^{*} h_{s}^{*}\right) \cdot S t \cdot k \cdot Q_{n}}{\Pi_{0}\left(f_{n}^{2} / f^{2}-1\right)}\left\{\cos \left(2 \pi t^{*}\right)-\cos \left(\omega_{n} t^{*}\right)\right\},
$$

which means that there is an amplification factor of $1 /\left(f_{n}^{2} / f^{2}-1\right)$ depending on the ratio between the natural frequency $f_{n}$ of the beam and the excitation frequency $f$. The full solution is $w^{*}\left(x^{*}, t^{*}\right)=h\left(t^{*}\right)+\sum_{n=1}^{\infty} X_{n}\left(x^{*}\right) T_{n}\left(t^{*}\right)$. The amplitude of the tip deformation, $\gamma$, for the first mode $(n=1)$ is given as

$$
\gamma=\frac{\left(1+\frac{4}{\pi} \rho^{*} h_{s}^{*}\right) \cdot S t \cdot k}{\Pi_{0}\left(f_{1}^{2} / f^{2}-1\right)}
$$

relative to the imposed rigid body motion normalized by the chord. The parameter $\gamma$ can be rewritten as

$$
\frac{\gamma}{h_{a} / c_{\mathrm{m}}}=\left(\frac{\rho_{f} c_{\mathrm{m}}}{\rho_{s} h_{s}} \frac{\pi}{4}+1\right) \frac{4}{\left(\frac{f_{1}}{f}\right)^{2}-1} \sim \frac{A+1}{\left(\frac{f_{1}}{f}\right)^{2}-1},
$$

where $f_{1} / f=\omega_{1} /(2 \pi)$ the inverse frequency ratio and $A=\pi \rho_{f} c_{\mathrm{m}} /\left(4 \rho_{s} h_{s}\right)$ is the ratio between the accelerationreaction force (added mass) and the wing inertia. Depending on the order of this ratio either the acceleration-reaction force term or the wing inertia force can be neglected. Equation (40) gives the relative wing tip deformation normalized by the plunge amplitude, which can be related to the Strouhal number based on the deformed tip displacement. Note that when $A$ is sufficiently large, the inertia force term can be neglected and $\gamma$ is then proportional to $A h_{a}^{*} \sim \rho_{f} h_{a} /\left(\rho_{s} h_{s}\right)$.

The proposed scaling parameter to estimate the resulting force on the flapping wing follows the observation that there exists a correlation between the dynamic deformation of the wing at the tip, $\gamma$, given by Eq. (39), and the static tip deflection which is $\left\langle C_{F}\right\rangle / \Pi_{1}$. To consider the non-dimensional energy equation, first multiply Eq. (24) with the relative wing velocity $\partial v^{*} / \partial t^{*}$ yielding, 


$$
\frac{\partial v^{*}}{\partial t^{*}} \Pi_{0} \frac{\partial^{2} v^{*}}{\partial t^{* 2}}+\frac{\partial v^{*}}{\partial t^{*}} \Pi_{1} \frac{\partial^{4} v^{*}}{\partial x^{* 4}}=\frac{\partial v^{*}}{\partial t^{*}} F\left(t^{*}\right)
$$

where for simplicity the external force acting on the wing is abbreviated with $F\left(t^{*}\right)$. Substituting the separation variables, $v\left(x^{*}, t^{*}\right)=X\left(x^{*}\right) T\left(t^{*}\right)$, considering only the first mode, in Eq. (41) gives

$$
\Pi_{0} \dot{T} \ddot{T} X^{2}+\Pi_{1} \dot{T} T X X^{\prime \prime \prime \prime}=F\left(t^{*}\right) \dot{T} X,
$$

where $\left({ }^{\circ}\right)$ denotes the time derivative and ()$^{\prime}$ the spatial derivative. Integrating Eq. (42) in $x$ from the wing root to the tip results in

$$
\Pi_{0} \dot{T} \ddot{T} \int_{0}^{1} X^{2} d x^{*}+\Pi_{1} \dot{T} T \int_{0}^{1} X X^{\prime \prime \prime \prime} d x^{*}=F(t) \dot{T} \int_{0}^{1} X d x^{*} .
$$

The second integral can be partially integrated using the boundary conditions to

$$
\int_{0}^{1} X X^{\prime \prime \prime \prime} d x^{*}=k_{1}^{4} \int_{0}^{1} X^{2} d x^{*}=k_{1}^{4}=\frac{\Pi_{0}}{\Pi_{1}} \omega_{1}^{2}
$$

where the normalization proporty of $X$, Eq. (32), is used. Inserting Eq. (44) into Eq. (43) yields,

$$
\Pi_{0} \dot{T} \ddot{T}+\Pi_{1} \dot{T} T k_{1}^{4}=F\left(t^{*}\right) \dot{T} Q_{1}
$$

where $Q_{1}=\int_{0}^{1} X d x^{*}$ as before, see Eq. (31). Now, Eq. (45) can be integrated as

$$
\Pi_{0} \dot{T} \mathrm{~d} \dot{T}+\Pi_{1} k_{1}^{4} T \mathrm{~d} T=Q_{1} F \mathrm{~d} T,
$$

or,

$$
\mathrm{d}\left(\frac{1}{2} \Pi_{0} \dot{T}^{2}+\frac{1}{2} k_{1}^{4} \Pi_{1} T^{2}\right)=\mathrm{d}\left(Q_{1}\left\langle C_{F}\right\rangle a T\right),
$$

where we have assumed that there exists a time-averaged value $\left\langle C_{F}\right\rangle$ with corresponding proportinality value $a$, such that

$$
F \mathrm{~d} T \approx a\left\langle C_{F}\right\rangle \mathrm{d} T=\mathrm{d}\left(a\left\langle C_{F}\right\rangle T\right) .
$$

The value $a$ is approximated as a constant in this study, however in general $a$ depends on time. Integration of Eq. (47) gives the energy balance

$$
\frac{1}{2} \Pi_{0} \dot{T}^{2}+\frac{1}{2} k_{1}^{4} \Pi_{1} T^{2}-Q_{1} a\left\langle C_{F}\right\rangle T=C,
$$

where $C$ is an integration constant that will be determined. The first term in Eq. (49) is the kinetic energy, the second the strain energy, and the third term the work done by the external force on the wing. From kinematic relations when $\dot{T}=0$, the relative tip displacement is at maximum position, i.e. $T=T_{\max }$, corresponding to $v_{\text {tip,max }}^{*}$. On the other hand, if the displacement is at the neutral position, we have the maximum velocity in free-vibration $\dot{T}_{\max }$ with

$$
\frac{1}{2} \Pi_{0} \dot{T}_{\max }^{2}=C
$$

Hence the energy balance Eq. (49) can be rewritten as 


$$
\frac{1}{2} \Pi_{1} k_{1}^{4} T_{\max }^{2}-Q_{1} a\left\langle C_{F}\right\rangle T_{\max }=\frac{1}{2} \Pi_{0} \dot{T}_{\max }^{2} .
$$

Using the previously determined solution Eq. (38) the maximum relative tip displacement and the velocity can be approximated as

$$
T_{\max } \approx b_{1} \gamma Q_{1}
$$

with $b_{1}$ again approximated as being some constant and

$$
\dot{T}_{\max }=\left\{\begin{array}{lll}
\gamma \cdot 2 \pi Q_{1} & \text { if } 2 \pi \gg \omega_{1}, & \text { i.e. } f_{1} \ll f \\
\gamma \cdot \omega_{1} Q_{1} & \text { if } 2 \pi \ll \omega_{1}, & \text { i.e. } f_{1} \gg f .
\end{array}\right.
$$

In the most situations the motion frequency $\omega$ is lower than the natural frequency of the wing $\omega_{1}$, hence with some other constant $b_{2}$ not necessarily equal to $b_{1}$, we can approximate the maximum velocity as

$$
\dot{T}_{\max } \approx b_{2} \omega_{1} \gamma Q_{1}
$$

Inserting the approximations Eq. (52) and Eq. (54) into the energy balance Eq. (49) gives

$$
\frac{1}{2} \Pi_{1} k_{1}^{4} b_{1}^{2} \gamma^{2}-a\left\langle C_{F}\right\rangle b_{1} \gamma=\frac{1}{2} \Pi_{0} b_{2}^{2} \omega_{1}^{2} \gamma^{2},
$$

which can be rewritten as

$$
a\left\langle C_{T}\right\rangle b_{1}=\frac{\Pi_{1} k_{1}^{4}}{2}\left(b_{1}^{2}-b_{2}^{2}\right) \gamma .
$$

Finally, by factoring all constants in Eq. (56) into some constant $c$, the resulting relation between the timeaveraged force $\left\langle C_{F}\right\rangle$ and the maximum relative tip displacement represented with the scaling factor $\gamma$ is

$$
\frac{<C_{F}>}{\Pi_{1}}=c \gamma .
$$

The resulting scaling, Eq. (57) for the three canonical cases are shown in Figure V-16. The nonlinearity exhibited in Figure V-16(a) is due to the approximations made for the constant $c$ which may be a function of $\gamma$ or time. For the chordwise flexible airfoils both $\gamma$ and the normalized force are significantly greater than other cases. When plotted in the log-scale, see Figure V-16(b), the scaling for all cases considered becomes more evident. A linear fit on the data set with the coefficient of determination of $R^{2}=0.98$ indicates that the relation between the normalized force and $\gamma$ is a power law with the exponent of 1.19. The relation originating from the dimensional analysis, Eq. (5), then simplifies to

$$
\left\langle C_{F}\right\rangle=\Pi_{1} \Psi(\gamma)
$$

with $\Psi(\gamma)=10^{0.98} \gamma^{1.19}$. The elastoninertial number, $\mathcal{N}_{\mathrm{ei}}$ that Thiria and Godoy-Diana [25] proposed as the thrust scaling parameter in air is a special case of $\gamma$, i.e.

$$
\gamma \stackrel{\rho^{*} h_{s}^{*} \gg 1 \text { and } f / f_{1} \gg 1}{\longrightarrow} \mathcal{N}_{\mathrm{ei}} .
$$

Important to note is that the $y$-axis in Figure $\mathrm{V}-16$ shows $\left\langle C_{F}\right\rangle$. Recall that $\left\langle C_{F}\right\rangle$ was defined as the force acting normal on the wing that is responsible for the wing deformation, hence $\left\langle C_{F}\right\rangle$ is normal to $\left\langle C_{T}\right\rangle_{\text {or }}\left\langle C_{L}\right\rangle$ depending on the direction of the wing deformation. For the purely plunging chordwise flexible airfoil cases in forward flight in water $\left\langle C_{F}\right\rangle=\left\langle C_{T}\right\rangle /(S t \cdot k)$ where the factor $S t \cdot k$ is the ratio between $C_{L, \max } /\left\langle C_{T}\right\rangle \sim S t \cdot k$ for the added mass force and $\left\langle C_{T}\right\rangle$ by [68] for purely plunging airfoils. For all chordwise flexible airfoil cases parametrized by $\left(f, h_{S}^{*}\right)$, 
$\left\langle C_{F}\right\rangle / \Pi_{1}$ shows almost a linear correlation with $\gamma$. Recall that the inertial force term arising from the plunging boundary condition is small compared to the added mass term because for the plunging chordwise flexible airfoils $\rho_{s}^{*} h_{s}^{*} \ll 1$. Compared to the higher motion frequency cases, the thrust generation at the lowest frequency at the five thicknesses shows larger variance, which are not shown in Figure V-16. A plausible explanation is that the current analysis breaks down due to the presence of the rigid teardrop at lower motion frequencies. When the plunging motion is very slow the rigid teardrop the large leading edge radius will produce time-averaged drag which overwhelms the thrust generation from the thin flat plate with small deflection. The airfoil produces drag at $S t=$ 0.085 on all five thickness ratios as shown in Figure V-4, which would result in underpredicted value of $\left\langle C_{F}\right\rangle / \Pi_{1}$.

For the spanwise flexible wing, although the Reynolds number and the thrust direction relative to the wing flexibility is different compared to the chordwise flexible airfoil, similar analysis could be made by approximating the three-dimensional wing as a beam with the correction factors $L / c_{\mathrm{m}}=A R$ for $\Pi_{0}$ and $\Pi_{1}$ as discussed in Section II. The force coefficient is scaled with the same parameters as for the chordwise flexible airfoils for the same reasons, i.e. $\left\langle C_{F}\right\rangle=\left\langle C_{T}\right\rangle /(S t / k)$. The time-averaged thrust coefficient from the numerical computation of the two flexibilities for different motion frequencies fall on top of the previous scaling obtained for the chordwise flexible airfoils.

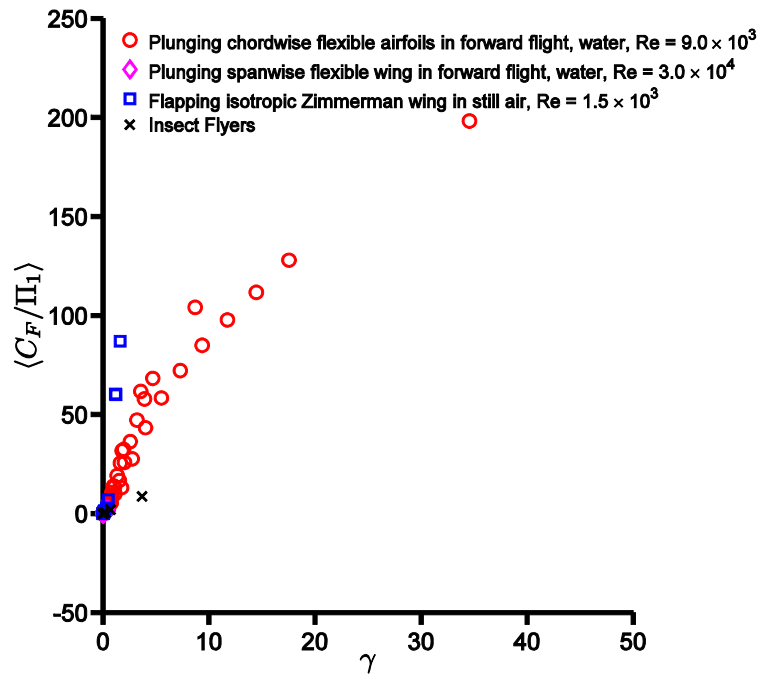

(a) linear scale

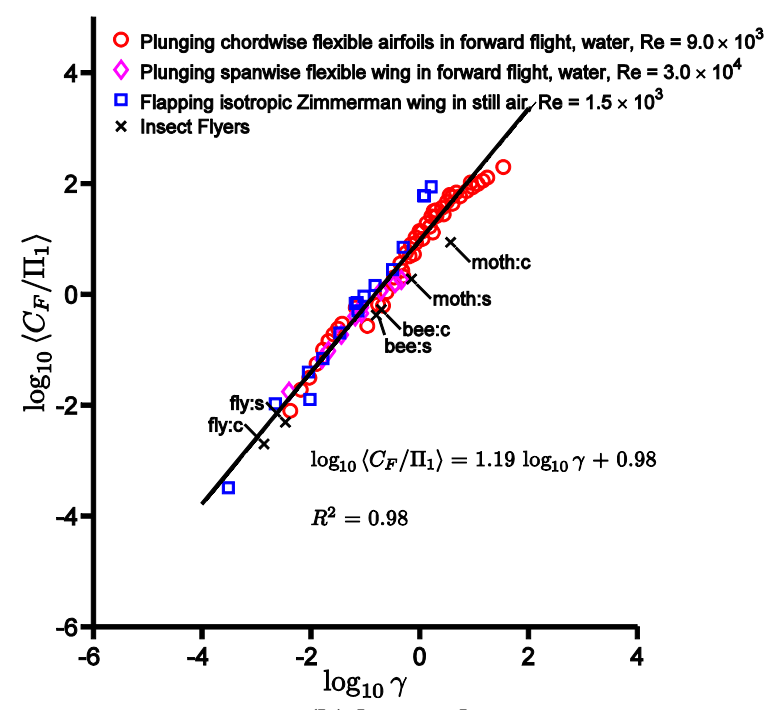

(b) log-scale

Figure V-16 Normalized time-averaged force coefficients as function of $\gamma$. For the insect flyers the letter $c$ and s correspond to chordwise and spanwise flexibility directions, respectively.

Because the wing is hovering in air for the case of flapping Zimmerman wing, the density ratio is higher than in water. Hence, the inertial force dominates over the added mass force as previously found [70,71]. The horizontal force $\left\langle C_{F}\right\rangle$ is found by normalizing $\left\langle C_{L}\right\rangle$ by $h_{S}^{*}$ because the vertical force and the horizontal force are proportional to the thickness ratio, if we assume that the pressure differentials are of the order of $O(1)$. Although this is a simplification, for the sixteen training points the thickness ratio scaling is confirmed by taking the ratio between the maximum $C_{L}$ and the maximum $C_{T}$ within different motion cycles for all cases as shown in Figure $\mathrm{V}-17$, which indeed show that the pressure differentials are of the order of $O(1)$. Furthermore, the computed lift from the numerical framework represents only the fluid dynamic force without the inertial force of the wing. The inertial force that acts on the wing is estimated by multiplying the factor $\rho^{*} h_{s}^{*} /(S t / k)$, which is the ratio between the inertial force $\left(\sim \rho^{*} h_{S}^{*} k^{2}\right)$ and the fluid force $(\sim S t k)$ to $\left\langle C_{L}\right\rangle$. The resulting normalization for the vertical axis is then $\left\langle C_{F}\right\rangle=\left\langle C_{L}\right\rangle \rho^{*} /(S t / k)$. 


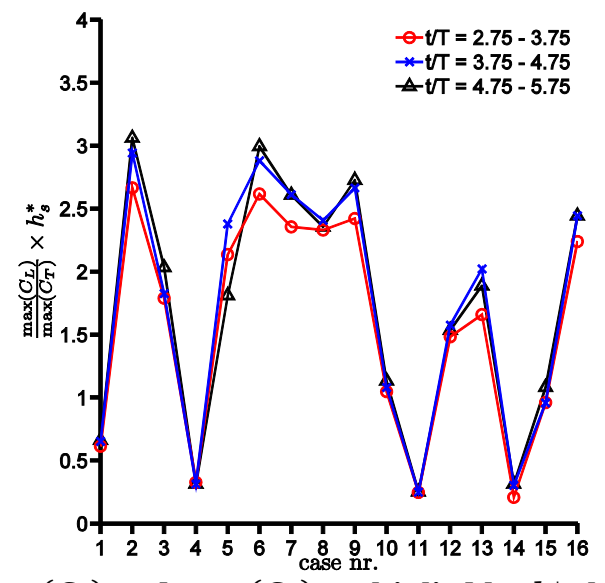

Figure V-17 Ratio between $\max \left(C_{L}\right)$ and $\max \left(C_{T}\right)$ multiplied by $h_{s}^{*}$ showing the order of magnitudes of the pressure differentials acting on the flapping Zimmerman wing hovering in air.

Even though the current case has different kinematics (plunging vs. flapping; forward flight vs. hover), different density ratio (low vs. high), and structural flexibilities (unidirectional vs. isotropic), the previous trend reemerges, suggesting the generality of this scaling parameter $\gamma$. The trends for the flapping isotropic Zimmerman wing hovering case is slightly offset in the vertical direction suggesting that the resulting lift is lower. An important aspect is influence of the presence of the rigid triangle (see Figure V-10) that constraints the tip deformation, such that the resulting tip deformation is less than the setup where the imposed kinematics is actuated at the root of the wing without the rigid triangle.

Table V-3 Kinematic, geometric, fluid, and structural parameters for the hawkmoth, bumble bee, and fruit fly obtained from the literature [73,74,75,76,77,78].

\begin{tabular}{cccc}
\hline \hline Insect & Hawkmoth & Bumble bee & Fruit fly \\
\hline$c_{\mathrm{m}}[\mathrm{mm}]$ & 18.2 & 3.22 & 0.96 \\
$R[\mathrm{~mm}]$ & 47.3 & 10.9 & 3.0 \\
$\omega /(2 \pi)[\mathrm{Hz}]$ & 26.1 & 181 & 240 \\
$\phi_{a}[\mathrm{deg}]$ & 57.2 & 72 & 75 \\
$\operatorname{Re}\left[10^{3}\right]$ & 6.2 & 2.2 & 0.25 \\
$k$ & 0.30 & 0.18 & 0.19 \\
$S t$ & 0.25 & 0.25 & 0.25 \\
$h_{s}^{*}\left[10^{-3}\right]$ & 2.0 & 1.0 & 0.6 \\
$\rho^{*}\left[10^{3}\right]$ & 2.0 & 2.1 & 1.1 \\
$\Pi_{1, \mathrm{~s}}\left[10^{2}\right]$ & 0.43 & 1.4 & 26 \\
$\Pi_{1, \mathrm{c}}$ & 0.53 & 2.8 & 211 \\
\hline \hline
\end{tabular}

For the flapping isotropic Zimmerman wing case in hover, we could correlate the lift generation to $\gamma$. This result suggests extrapolation of the current scaling analysis for the lift generation of hovering insect flyers. The lift, in hover, for several insects is approximated as the experimentally measured weights of hawkmoth [73,74], bumble bee [75], and fruit fly [76,77]. To calculate the parameters listed in Table V-3 flapping rectangular planform with constant thickness has been assumed with constant thickness and density. To compute the effective stiffnesses in the spanwise and the chordwise directions, i.e. $\Pi_{1, \mathrm{~s}}$ and $\Pi_{1, \mathrm{c}}$, respectively, the flexural stiffness data presented by [78] along with their wing lengths have been used. The result is included in Figure V-16 with the scaling

$$
\frac{W}{\frac{1}{2} \rho_{f} U_{\mathrm{ref}}^{2} c_{\mathrm{m}}^{2}}=\Pi_{1} \frac{S t / k}{\rho^{*} h_{s}^{*}} \Psi(\gamma) \sim \Pi_{1} \frac{\rho_{f} h_{a}}{\rho_{s} h_{s}} \Psi(\gamma) .
$$

Again, the lift approximated with the weights of the insects scales with $\gamma$. 


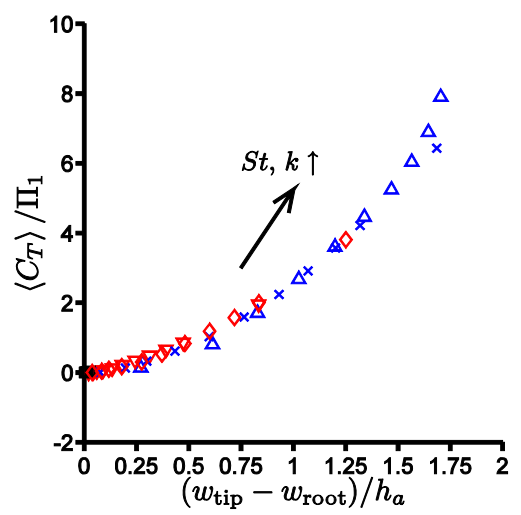

(a) Chordwise flexible:

$\Delta h_{s}^{*}=0.56 \times 10^{-3}$;

$\times h_{s}^{*}=0.85 \times 10^{-3}$;

$\diamond h_{s}^{*}=1.13 \times 10^{-3}$;

$\nabla h_{s}^{*}=1.41 \times 10^{-3}$

$\square h_{s}^{*}=4.23 \times 10^{-3}$

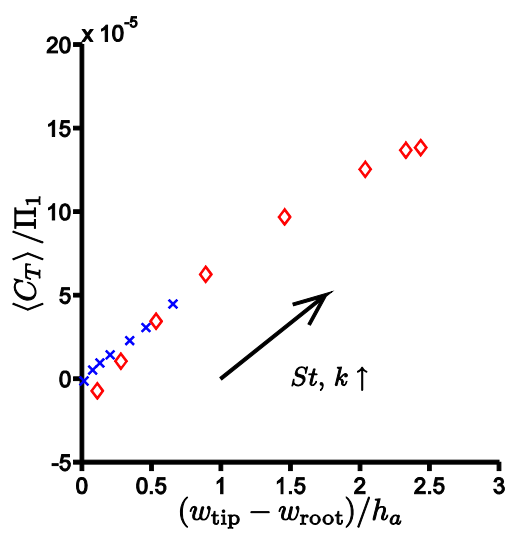

(b) Spanwise flexible:

$\times \Pi_{1}=121, \rho^{*}=7.8$

$\diamond \Pi_{1}=38, \rho^{*}=2.7$

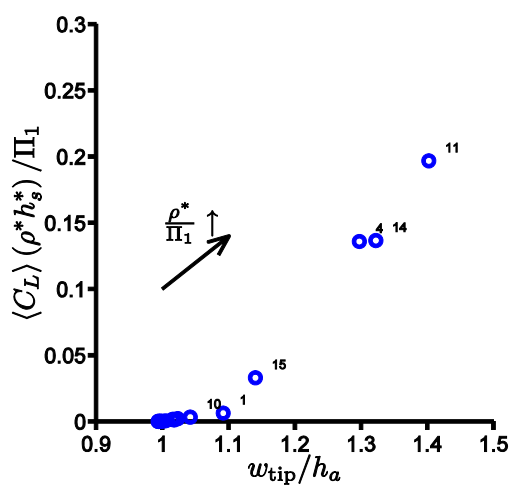

(c) Isotropic Zimmerman

Figure V-18 Time-averaged force (thrust or lift) coefficient normalized by the effective stiffness plotted against the maximum relative tip deformations for the current computations. The numbers shown in (c) next to the markers indicate their case numbers

The current analysis shows that the time-averaged force, such as the thrust or lift, can be related to the maximum relative tip displacement by normalizing the force by the effective stiffness, $\Pi_{1}$, resulting in a measure equivalent to the static tip displacement. Figure V-18 illustrates the static displacement, $\left\langle C_{T}\right\rangle / \Pi_{1}$, as function of the relative tip displacement for all thicknesses and motion frequencies considered. The results for the five different thickness ratios collapse to a single curve with higher motion frequency leading to greater thrust. Note that the relation is not linear, indicating that $c$ in Eq. (57) is actually not a constant. For the spanwise flexible wing case, although the Very Flexible wing has larger relative deformation, the effective stiffness is 5.6 times smaller than that of the Flexible wing. Hence even if the static tip displacement is larger for the Very Flexible wing at the highest motion frequency, the force corresponding to this deformation is smaller (see Figure V-8 due to smaller effective stiffness, see Figure V-18. Furthermore, again, plotting the $\left\langle C_{T}\right\rangle / \Pi_{1}$ against the maximum relative tip deformation collapses both curves on top of each other. It is shown in Eq. (28) that for $\rho^{*} h_{s}^{*} \gg 1$ the inertial force will have greater influence on the wing deformation than the force due to added mass. In air, $\rho^{*}$ is high, so that in addition to the normalization by the effective stiffness, the time-averaged force needs to be multiplied with $\rho^{*} h_{s}^{*}$ to account for the inertial force. Figure V-18 again shows that the time-averaged force, in this case the lift of the flapping isotropic Zimmerman wing hovering, can be scaled with the maximum relative tip displacement by properly normalizing the force.

\section{V.2 Propulsive Efficiency of Flapping Flexible Wings}

The propulsive efficiency defined as

$$
\eta=\frac{\left\langle C_{T}\right\rangle}{\left\langle C_{P}\right\rangle}
$$

where $\left\langle C_{P}\right\rangle$ is the time-averaged power input for purely plunging wing computed as

$$
\left\langle C_{P}\right\rangle=\left\langle C_{L} \dot{h}\right\rangle .
$$

Note that the time-averaged power due to inertia vanishes for sinusoidal motions [64] since

$$
\left\langle C_{P, \text { inertia }}\right\rangle \sim\langle\ddot{h} \dot{h}\rangle=\int_{0}^{1} \ddot{h} \dot{h} d t \sim \int_{0}^{1} \cos (2 \pi t) \sin (2 \pi t) d t=0 .
$$


The propulsive efficiency for the chordwise flexible airfoils described in Section V.1.A and the spanwise flexible wings in Section V.1.B are plotted against the $S t$ in Figure V-19. For comparison purposes the experimental measurements $[64,65]$ for both cases are included. For the chordwise flexible airfoils the efficiency increases with decreasing $h_{s}^{*}$. Furthermore, the efficiency first increases with increasing motion frequency, i.e. St since the plunge amplitude is kept constant, however then plateaus reaching some optimal efficiency. The thinnest airfoil generates the highest $\eta$. The experimental measurements illustrated in Figure V-19(b) show a similar trend, however there is an offset compared to the computed values. Again, uncertainties involved in the computational modeling or experimental setup may play a role. Moreover, as it will be shown below, the magnitude of $\left\langle C_{P}\right\rangle$ is an order of magnitude smaller than $\left\langle C_{T}\right\rangle$, hence even a small uncertainty in the power input measurement will lead to large difference in the resulting $\eta$. For the spanwise flexible wings (Figure V-19(c, d)), similar trends are found.

Before discussing the scaling of the power input and the propulsive efficiency, the scaling for the thrust generation for the chordwise flexible airfoils and the spanwise flexible wings are summarized in Figure $\mathrm{V}-20$ and is $\left\langle C_{T}\right\rangle / \beta_{1} \sim \gamma^{1.17}$ with $\beta_{1}=\Pi_{1} /(k / S t)$. The power of $\gamma$ has changed slightly compared to the previously determined value of 1.19 (Figure V-16) because the data points from the isotropic Zimmerman wing cases and the insects are excluded. Although the value is different, the qualitative trend of the propulsive efficiency that will be discussed later will remain the same.

An interesting discussion on the power input arises from the fact that the fluid dynamic force has been modeled as added mass term, which is proportional to the acceleration of the wing motion. If the wing were rigid, then, similar to the power input due to the inertial force,

$$
\left\langle C_{P, \text { added mass, rigid }}\right\rangle \sim\langle\ddot{h} \dot{h}\rangle=0,
$$
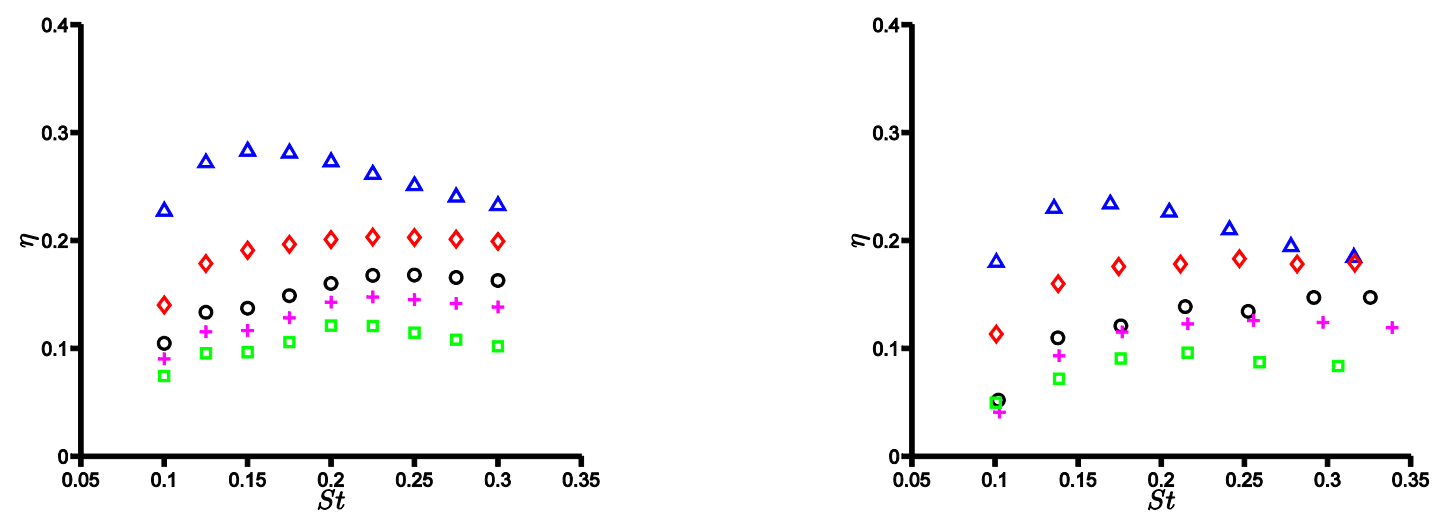

$\Delta h_{s}^{*}=0.56 \times 10^{-3} ; \diamond h_{s}^{*}=0.85 \times 10^{-3} ; \circ h_{s}^{*}=1.13 \times 10^{-3} ;+h_{s}^{*}=1.41 \times 10^{-3} ; \square h_{s}^{*}=4.23 \times 10^{-3}$

(a) chordwise flexible airfoil, computation

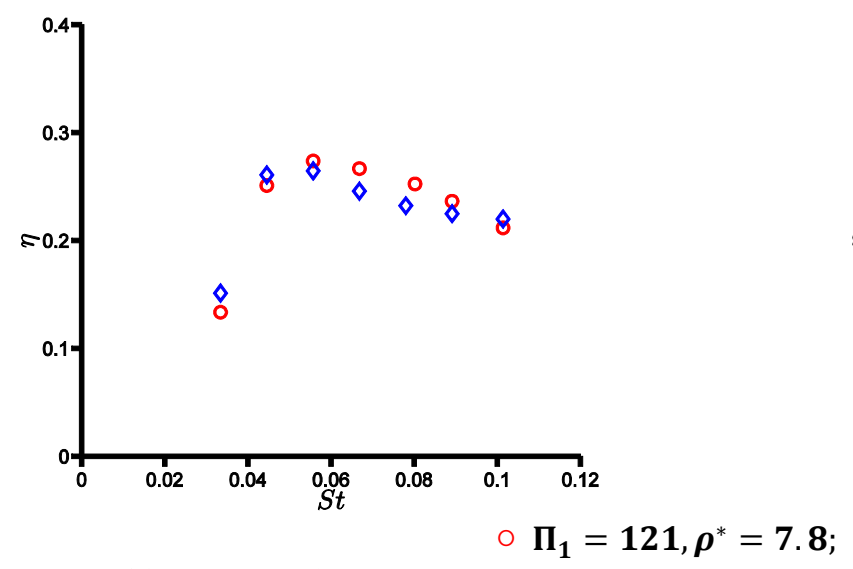

(c) spanwise flexible wing, computation

(b) chordwise flexible airfoil, experimental measurement extracted from [64]

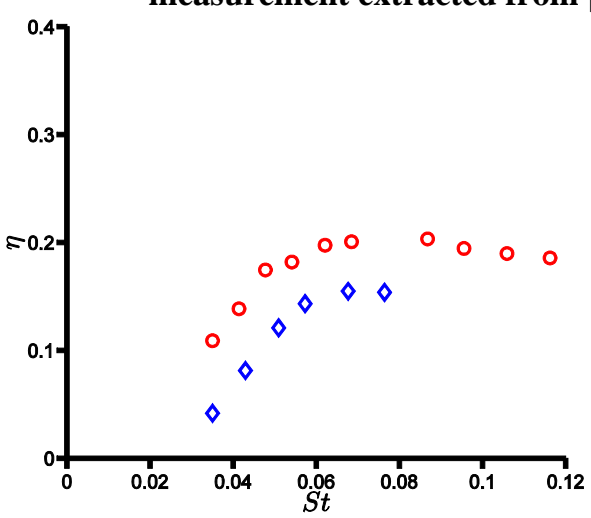

$\Pi_{1}=38, \rho^{*}=2.7$

(d) spanwise flexible wing, experimental measurement extracted from [65]

Figure V-19 Propulsive efficiency plotted against the Strouhal number for the chordwise flexible airfoil cases (a) - (b) and the spanwise flexible wing cases (c) - (d). 


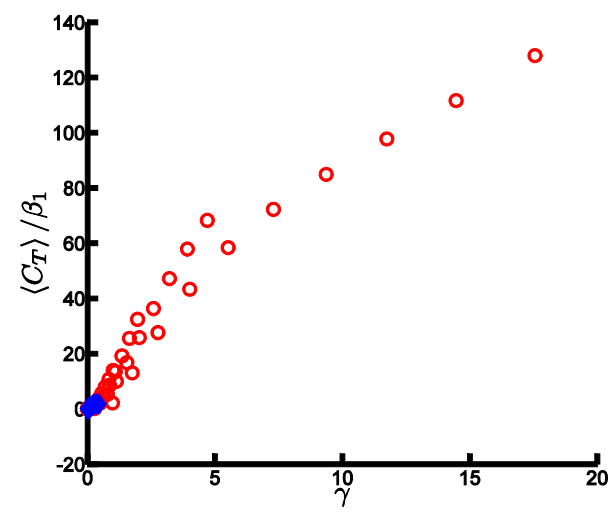

(a) linear scale

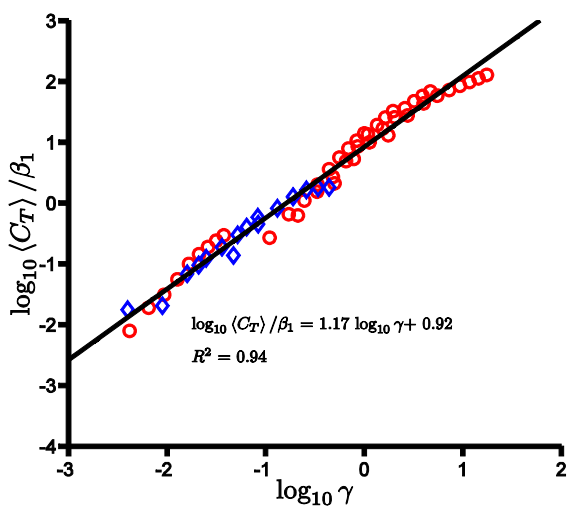

(b) log-scale

Figure V-20 Time-average thrust scaling plotted against $\gamma . \circ$ : chordwise flexible airfoils; $\diamond$ : spanwise flexible wings.

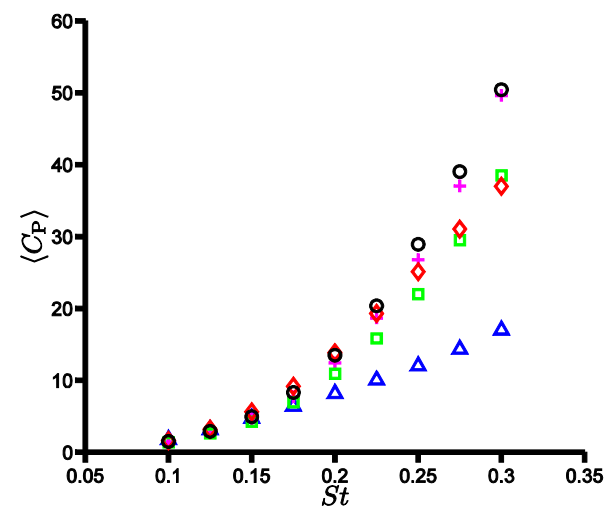

(a) chordwise flexible airfoil:

$\Delta h_{s}^{*}=0.56 \times 10^{-3}$

$\diamond h_{s}^{*}=0.85 \times 10^{-3}$;

$\circ h_{s}^{*}=1.13 \times 10^{-3}$

$+h_{s}^{*}=1.41 \times 10^{-3}$

$\square h_{s}^{*}=4.23 \times 10^{-3}$

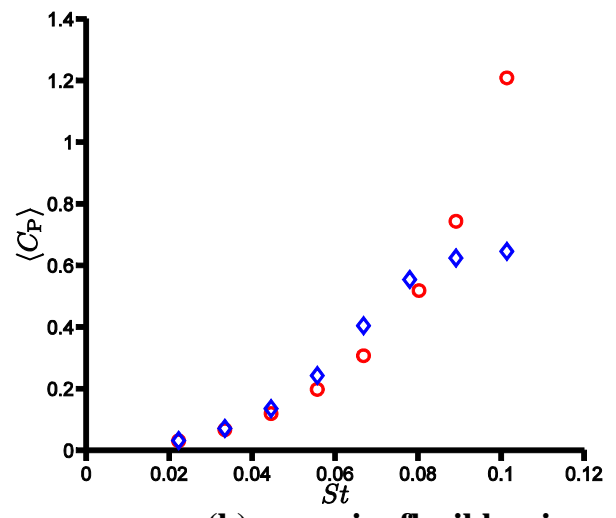

(b) spanwise flexible wing

$\circ \Pi_{1}=121, \rho^{*}=7.8$

$\diamond \Pi_{1}=38, \rho^{*}=2.7$

Figure V-21 Time-average power input plotted against the Strouhal number (St)

which is clearly not the case, see Figure V-21. For small $S t$ the power input scales as $S t^{2}$, however as the $S t$ increases either the thickness ratio for the chordwise flexible airfoils, or the different structural properties for the spanwise flexible wings affect the resulting power. That the power required is non-zero, means that the resulting instantaneous lift on the wing should have a phase lag relative to the imposed motion. A major source for the phase lag is due to the wing deformation. By acknowledging for the wing deformation given in Eq. (38), the time-averaged power input coefficient due to added mass can be approximated as in the first mode

$$
\left\langle C_{P, \text { added mass }}\right\rangle=\int_{0}^{1} \Pi_{0}(\ddot{T}+\ddot{h}) \dot{h} d t=\pi^{2} \frac{Q_{1} \gamma^{2} k_{1}^{8} \Pi_{1}^{2}}{k^{2}\left(1+\frac{4}{\pi} \rho^{*} h_{s}^{*}\right)} \sim \frac{\Pi_{1}^{2}}{k^{2}\left(1+\frac{4}{\pi} \rho^{*} h_{s}^{*}\right)} \gamma^{2}=\beta_{2} \gamma^{2}
$$

where the integral is approximated as

$$
\int_{0}^{1} \cos \left(\omega_{1} t\right) \sin (2 \pi t) d t=\frac{2 \pi\left(1+\cos \omega_{1}\right)}{\omega_{1}^{2}-4 \pi^{2}} \approx-\frac{\pi \omega_{1}^{2}}{\omega_{1}^{2}-4 \pi^{2}}=-\frac{\Pi_{1}}{4 \pi} \frac{\gamma}{S t k\left(1+\frac{4}{\pi} \rho^{*} h_{s}^{*}\right)} .
$$


For $\Pi_{1} \gg \Pi_{0}$ the scaling for $\left\langle C_{P}\right\rangle$ reduces to $S t^{2}\left(1+4 \rho^{*} h_{S}^{*} / \pi\right)$, hence in water, such as in the experimental setup considered in this case $[64,65]$ or for fixed density ratios and thickness ratios of the wing the $\left\langle C_{P}\right\rangle \sim S t^{2}$, consistent with the previous literature [65] [26] and Figure V-21.

Figure V-22(a) shows the time-averaged power input normalized by $\beta_{2}$ given in Eq. (65) against $\gamma^{2}$ for both chordwise flexible airfoil and spanwise flexible wing cases. As the scales of $\left\langle C_{P}\right\rangle$ vary enormously, $\left\langle C_{P}\right\rangle / \beta_{2}$ is plotted against $\gamma$ in log-scale. A linear fit with $R^{2}=0.98$ indicates that the power input scales with $\gamma^{2.13}$.

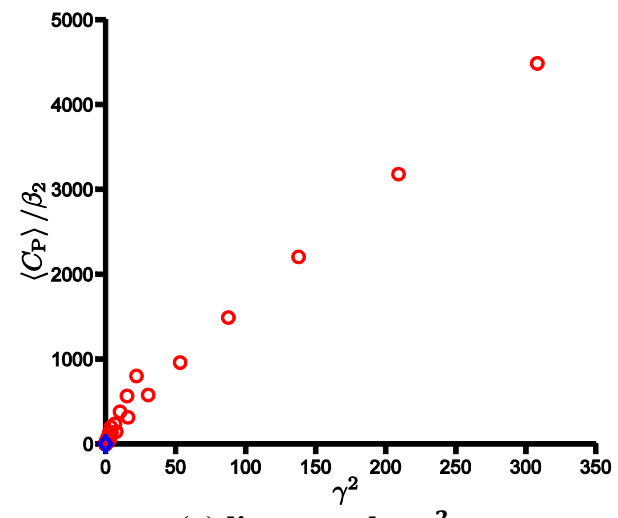

(a) linear scale: $\gamma^{2}$

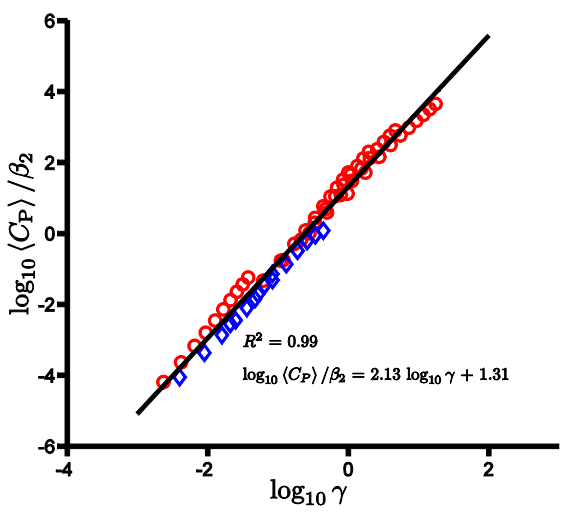

(b) log-scale: $\gamma$

Figure V-22 Time-average power input normalized by $\beta_{2}$ plotted against $\gamma$.

$\circ$ : chordwise flexible airfoils; $\diamond$ : spanwise flexible wings.

The scaling for the propulsive efficiency now follows from the scaling for the thrust, i.e. $\left\langle C_{T}\right\rangle / \beta_{1} \sim \gamma^{1.17}$ with $\beta_{1}=\Pi_{1} /(k / S t)$ and $\left\langle C_{P}\right\rangle / \beta_{2} \sim \gamma^{2}$ with $\beta_{2}=\frac{\Pi_{1}^{2}}{k^{2}\left(1+\frac{4}{\pi} \rho^{*} h_{s}^{*}\right)}$ as

$$
\eta=\frac{\left\langle C_{T}\right\rangle}{\left\langle C_{P}\right\rangle} \sim \frac{\beta_{1} \gamma^{1.17}}{\beta_{2} \gamma^{2}} \rightarrow \frac{\eta}{\beta_{3}} \sim \gamma^{1.17}
$$

where $\beta_{3}=\frac{S t k}{\Pi_{1}} \frac{\left(1+\frac{4}{\pi} \rho^{*} h_{S}^{*}\right)}{\gamma^{2}}$. The resulting scaling is shown in Figure V-23.

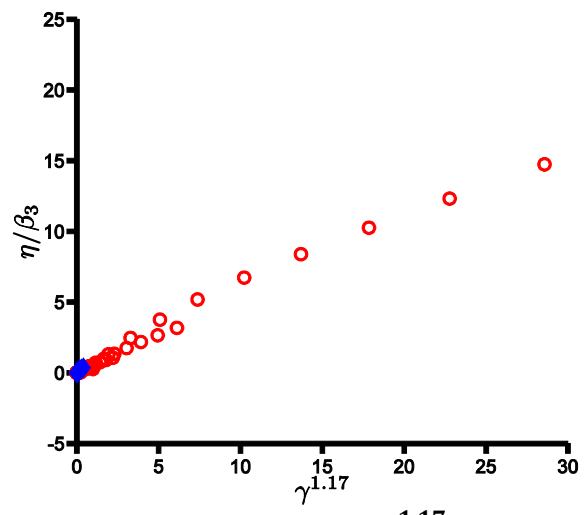

(a) linear scale: $\gamma^{1.17}$

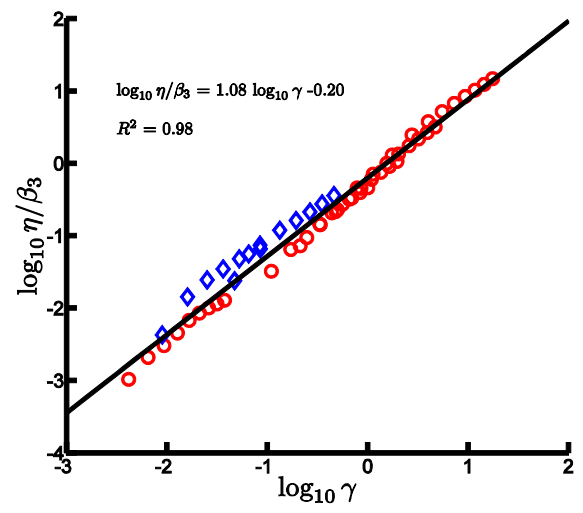

(b) log-scale: $\gamma$

Figure V-23 Propulsive efficiency normalized by $\beta_{3}$ plotted against $\gamma$. $\circ$ : chordwise flexible airfoils; $\diamond$ : spanwise flexible wings. 


\section{V.3 Implications of the Scaling Parameters on the Aerodynamic Performance of Flapping Flexible Wings}

The time-averaged force $\left\langle C_{F}\right\rangle$ and the propulsive efficiency $\eta$ could be related to the resultant force on the wing depending on the situation, such as fluid/inertial force, with/without freestream, or thrust/lift/weight. The current result enables us to estimate the order of magnitude of the time-averaged force generation and and its efficiency for a flexible flapping wing using a priori known parameters.

Furthermore, the scaling can guide design of flapping wing micro air vehilces. For example, to support a given weight $W_{\text {body }}$ of a vehicle body in air, the scaling Eq. (60) reduces to

$$
W_{\text {body }} \sim \frac{\rho_{s}^{1} R^{3.19} f^{2.38} \phi_{a}^{2.19} c_{\mathrm{m}}^{1.57}}{h_{s}^{0.38} E^{0.19}} \rightarrow f \sim \frac{m^{0.42} g^{0.42} h_{s}^{0.16} E^{0.080}}{\rho_{s}^{0.42} R^{1.34} \phi_{a}^{0.92} c_{m}^{0.66}}
$$

assuming $\Pi_{1} \gg \Pi_{0}$. The condition $\Pi_{1} \gg \Pi_{0}$ is satisfied when $f_{1} \gg f$ and simplifies the algebra, however it poses constraints on the range of the structural properties, such that the natural frequency of the wing is higher than the motion frequency. Relation Eq. (68) shows that either increasing the wing area, motion frequency, or the flapping amplitude helps to generate sufficient lift to sustain hover flight. On the other hand, by making the wing softer, i.e. reducing the Young's modulus or wing thickness, wing deformations will increase leading to higher lift generation. However, softening the wing further will violate the frequency ratio assumption: $f_{1} / f \gg 1$. The relation given for the flapping frequency has similar correlation as the one identified by Pennycuick [79]. Note that the wing weight is assumed to be negligible compared to the body weight in this discussion.

Another implication of the scaling is the interesting behaviour of the role of $h_{s}^{*}$ for the chordwise flexible airfoil cases shown in Section V.1.A. It was observed that the $\left\langle C_{T}\right\rangle$ increased first and then decreased with decreasing $h_{S}^{*}$, see Figure V-4. Using the current scaling, which is repeated as

$$
\left\langle C_{T}\right\rangle \sim \Pi_{1} \frac{S t}{k} \gamma^{1.17}=\Pi_{1} \frac{h_{a}}{c_{m}}\left\{\frac{S t k}{\Pi_{0}\left(\frac{k_{1}^{4}}{4 \pi^{2}} \frac{\Pi_{1}}{\Pi_{0}}-1\right)}\right\}^{1.17}
$$

consider first the situation that $h_{s}^{*}$ is large, i.e. $\Pi_{1} \gg \Pi_{0}$. Then the denominator in $\gamma$ can be approximated as $\Pi_{0}\left\{\frac{k_{1}^{4}}{4 \pi^{2}} \frac{\Pi_{1}}{\Pi_{0}}-1\right\} \approx \Pi_{1}$ yielding

$$
\left\langle C_{T}\right\rangle \sim S t^{2}\left(\frac{S t k}{\Pi_{1}}\right)^{0.17}
$$

hence by reducing the thickness ratio, $\Pi_{1} \sim h_{s}^{* 3}$ will decrease, leading to the observed enhanced $\left\langle C_{T}\right\rangle$. However, decreasing $h_{s}^{*}$ further, the frequency ratio $f_{1} / f$ will be eventually of the same order of magnitude, resulting in a different physical behaviour. If, say $f_{1} / f=O(1)$, but not in resonance region, then the denominator in $\gamma$ will scale as $\Pi_{0}\left\{\frac{k_{1}^{4}}{4 \pi^{2}} \frac{\Pi_{1}}{\Pi_{0}}-1\right\} \approx \Pi_{0}$. Then, the resulting scaling will be

$$
\left\langle C_{T}\right\rangle \sim S t^{2}\left(\frac{S t k}{\Pi_{0}}\right)^{0.17} \frac{\Pi_{1}}{\Pi_{0}}
$$

Since $\Pi_{1} \sim h_{s}^{* 3}$ and $\Pi_{0} \sim h_{s}^{*}$, we have $\left\langle C_{T}\right\rangle \sim h_{s}^{* 1.83}$. This is consistent with the trend shown in Figure V-4 that reducing the thickness ratio further, the thrust deterioates.

Furthermore, the thrust scaling for flapping flexible wings in water in forward motion, Eq. (69) can be rewritten as

$$
\left\langle C_{T}\right\rangle \sim S t^{2}\left(\frac{\rho_{f} h_{a}}{\rho_{s} h_{s}}\right)^{0.17}\left(\frac{f_{1}}{f}\right)^{2}\left\{\frac{1}{\left(\frac{f_{1}}{f}\right)^{2}-1}\right\}^{1.17}
$$


and since $S t \sim \omega, k \sim \omega$, and $\Pi_{0} \sim k^{2} \sim \omega^{2}$ the thrust has a maximum at the resonance frequency. In reality, there is damping in the system, either structural or aerodynamic [26]. Although the effects of damping is not considered in this study, applying the effects of damping for linear oscillators (e.g. [68]) yields the resonance frequency slightly below the natural frequency of the wing with finite value for the maximum relative wing tip deformation, hence the thrust, see Figure V-24(a, b). In Figure V-24(b) the effects of damping has been incorporated by approximating the denominator term as

$$
\sqrt{\left\{1-\left(\frac{f}{f_{1}}\right)^{2}\right\}^{2}+\left(d \frac{f}{f_{1}}\right)^{2}}
$$

where $d$ is some small damping coefficient. This finding is consistent with the previous findings that the optimal propulsive performance is found near the natural frequency of the wing, but slightly below [27,28,25,26].

The propulsive efficiency scaling Eq. (67) can be rewritten in terms of frequency ratio $f / f_{1}$ as

$$
\eta \sim\left\{1-\left(\frac{f}{f_{1}}\right)^{2}\right\}^{0.83}\left(\frac{f}{f_{1}}\right)^{0.34}
$$

which has a local maximum at $f=0.41 f_{1}$. Depending on the scaling of the force, the optimal frequency can be found as

$$
\frac{\partial \eta}{\partial f}=\left.0 \rightarrow \frac{f}{f_{1}}\right|_{\mathrm{opt}}=\sqrt{\beta-1}
$$

where $\beta$ is the exponent of $\gamma$ in the force scaling, i.e. 1.17 in Eq. (67), or 1.19 in Eq. (58) which yields the optimal frequency of 0.41 of the natural frequency. This indicates that the optimal efficiency is not achieved at the resonance, however the optimal frequency is some fraction of the natural frequency of the wing, which is also consistent with the previous findings in the literature $[33,34,80,25,26]$, see Table V-4. Figure V-24(c) which plots Eq. (74) as function of the frequency ratio shows that the efficiency increases with the increasing frequency ratio until the optimal efficiency and then drops to zero at the resonance frequency. Since the undamped linear oscillator is unable to represent the resonance behavior correctly, an arbitrary damping has to be included in the system as before. Figure V-24(b) shows the effects of including damping, where $d$ is taken as 0.0, 0.2, and 0.4: the optimal frequency ratio increases with increasing $d$ as well as the efficiency at the resonance frequency. Note also the similarity between Figure V-24(b) with the computed propulsive efficiency curves shown in Figure V-19. Finally, a qualitative comparison is shown in Figure V-25 where the propulsive force and efficiency as function of the frequency ratio is plotted based on the current scaling and the measurements reported in Ramananarivo, GodoyDiana, and Thiria. [26]. Although the precise detail is different, the overall qualitative trend is similar.

For an example of a $2 \%$ thickness wing with rectangular platform made of aluminum hovering in air, the optimal frequency of the flapping motion is $5.4 \mathrm{~Hz}$ when the wing has a chord length of $20 \mathrm{~cm}$ and span of $50 \mathrm{~cm}$ with a flapping amplitude of $30 \mathrm{deg}$. Scaling down the geometry of the wing ten times to the chord length of $2 \mathrm{~cm}$ and span of $5 \mathrm{~cm}$, keeping the aspect ratio the same, the optimal flapping frequency increases to $54 \mathrm{~Hz}$. The resulting propulsive force coefficient and the propulsive efficiency remain the same for both cases. However, the dimensional propulsive force and the power required will be 100 times smaller for the smaller sized wing, proportional to the square of the chord. On the other hand, for the same aspect ratio and the thickness ratio, the volume of the wing is proportional to cubic power of the chord. Consequently, for the same material, the mass of the smaller wing is 1000 times smaller. The current scaling shows, consistent with Shyy et al. [3] that smaller flyers need to flap faster from the efficiency point of view, but the relative payload capacity increases because its weight reduces at a much faster rate compared to a larger flyers. 


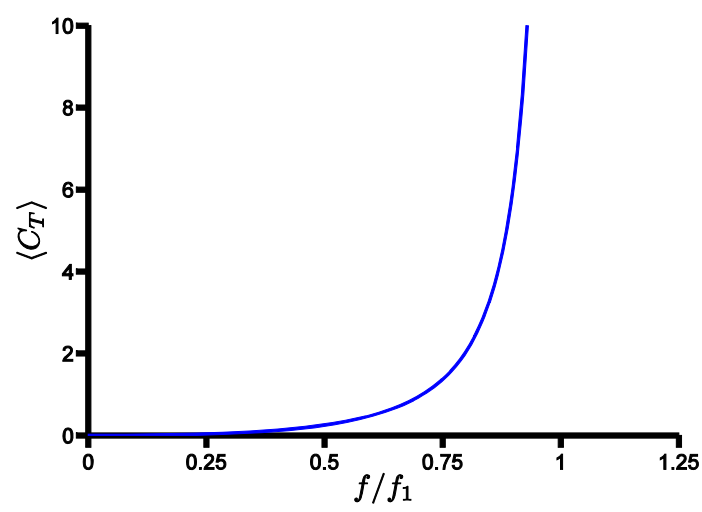

(a) undamped force

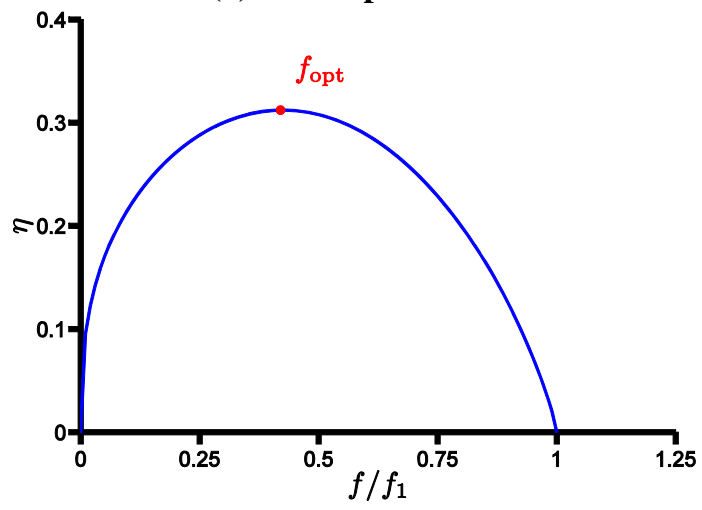

(c) undamped efficiency

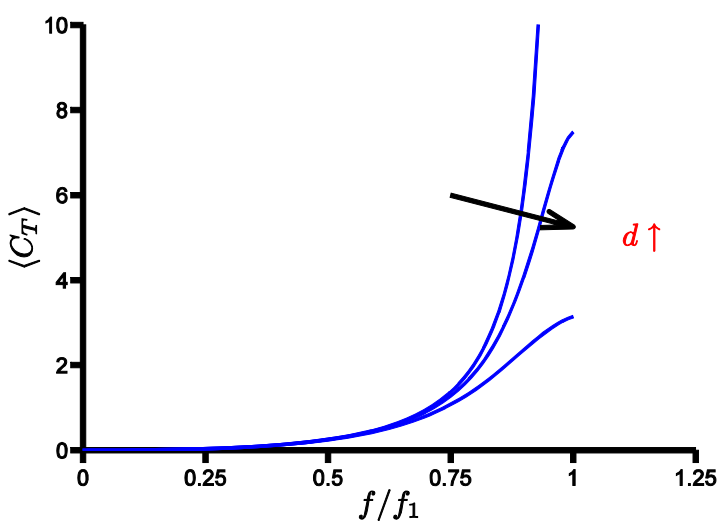

(b) force with damping

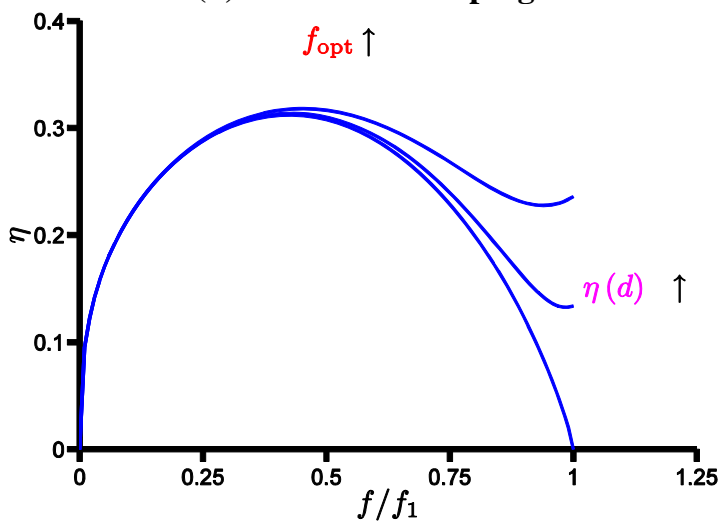

(d) efficiency with damping

Figure V-24 Force and propulsive efficiency plotted against the frequency ratio $f / f_{1}$.

Table V-4 Comparison of the optimal frequency ratios from the values reported in the literature and the current study.

\begin{tabular}{ccc}
\hline \hline Literature & $f / f_{1}$ & Description \\
\hline Vanella et al. [34] & 0.3 & Hover, 2D airfoil, Navier-Stokes equation solver coupled \\
with torsion spring model
\end{tabular}

Finally, the scaling parameters for diverse flow and kinematics conditions are summarized in Table V-5. For the forward flight in water the effective stiffness $\Pi_{1}$, the normalized plunge amplitude $h_{a}^{*}$, the mass ratio $\mu_{s}=$ $\rho_{f} h_{a} /\left(\rho_{s} h_{s}\right)$, and the frequency ratio $f / f_{1}$ dictate the propulsive force and the efficiency. For hover in air, for the studied kinematics, the role of the mass ratio is taken over by the factor $\rho^{*} h_{a}^{*}=\mu_{s} / h_{s}^{*}$. The efficiency for the hover motion in air is left as future work in this study and the resulting scaling is only predicted by following the same argument as for the propulsive efficiency in forward flight in air. 


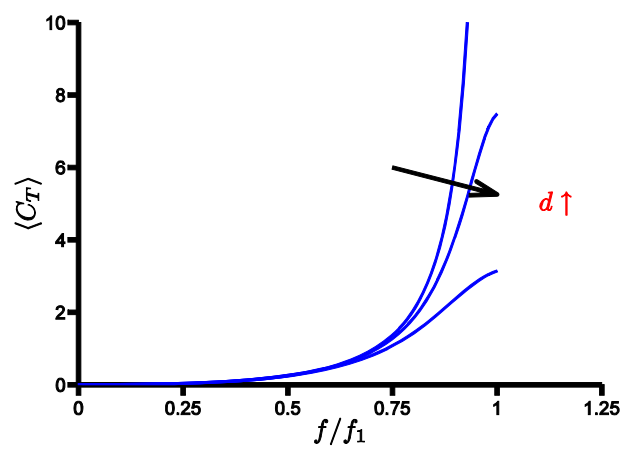

(a) propulsive force, current study

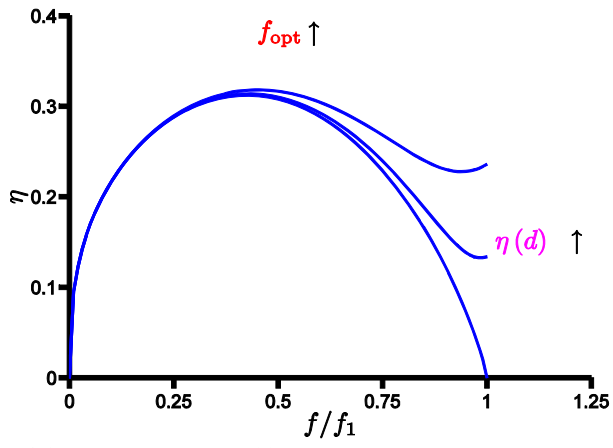

(c) propulsive efficiency, current study

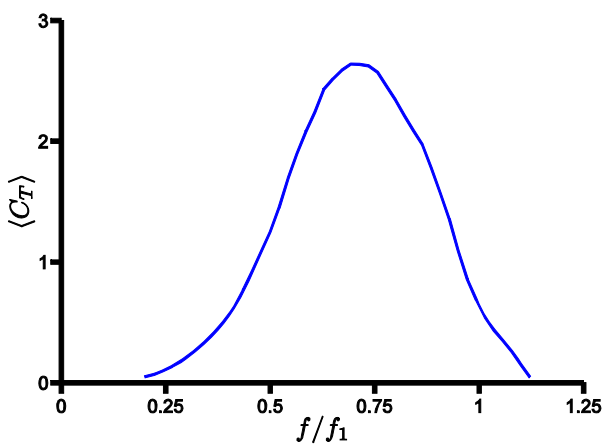

(b) propulsive force, data extracted from Ramananarivo, Godoy-Diana, and Thiria [26] for the thrust power $p_{T}$

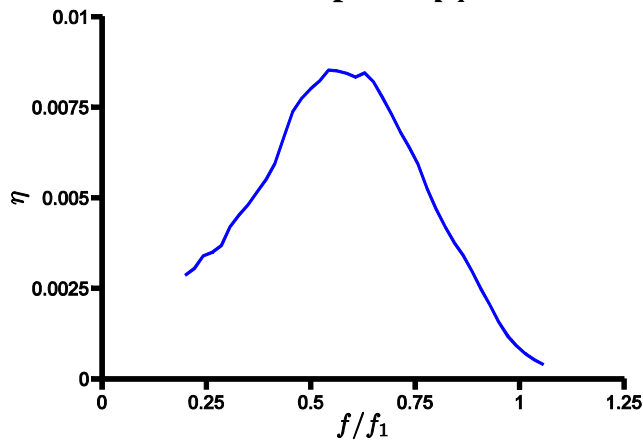

(d) efficiency interpolated from the thrust power $p_{T}$ and input power $p_{i}$ extracted from Ramananarivo, Godoy-Diana, and Thiria [26]

Figure V-25 Comparison of the propulsive force and efficiency from the current study and from the measurements obtained by Ramananarivo, Godoy-Diana, and Thiria [26].

Notice that the factor $\rho^{*} h_{a}^{*}=\rho_{f} h_{a} /\left(\rho_{s} c_{m}\right)$ is much smaller than $\mu_{s}=\rho_{f} h_{a} /\left(\rho_{s} h_{s}\right)$ hence resulting in a much lower propulsion for the hovering symmetric flap/plunge motion in air, since the thickness ratio is usually only of the order of 0.01. This order estimation matches well with the values shown in Ref. [26]. For the hovering Zimmerman wing the ratio between the propulsive force $C_{L}$ and the force required for input power, $C_{T}$, was of the order of $h_{s}^{*}$, see Figure V-17. This scaling suggests that the insects may require different mechanisms, such as active or passive pitching motion with reinforced leading edge, which is commonly observed in many insect wings. Furthermore, non-symmetric motions, such as figure- 8 motion where the wing experiences forward motion may yield higher efficiency [81]. With observations from experimental studies [78], anisotropic wing structures with different orders of flexibility may be essential to evince the high propulsive efficiency mechanism of insects.

Table V-5 Summary of the scaling proposed. Note $\beta_{1}$ is $\frac{\Pi_{1} S t}{k}$ for forward flight in water and $\frac{\Pi_{1} S t}{\rho^{*} k}$ for hovering flight in air, and $\beta_{3}$ is $\frac{S t k}{\Pi_{1}} \frac{\left(1+\frac{4}{\pi} \rho^{*} h_{s}^{*}\right)}{\gamma^{2}}$.

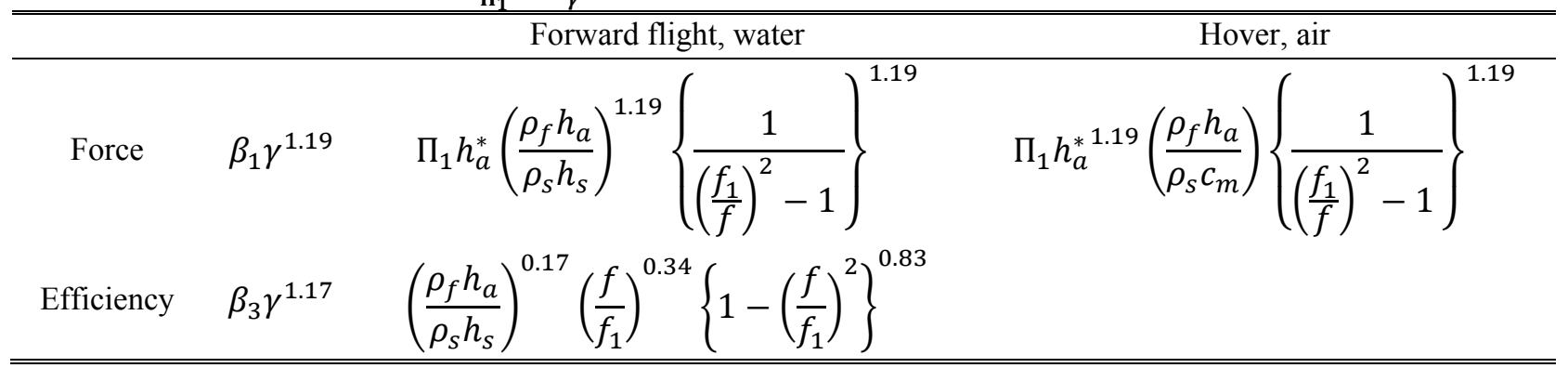




\section{Summary and Conclusions}

This study addresses modeling aspects of the fluid physics and fluid dynamic performances associated with flapping wings. Consistent dimensional analysis and non-dimensionalization of the governing equations for the fluid and the wing structure led to a system of non-dimensional parameters: Reynolds number $(R e)$, reduced frequency $(k)$, Strouhal number $(S t)$, aspect ratio $(A R)$, effective stiffness $\left(\Pi_{1}\right)$, effective angle of attack $\left(\alpha_{e}\right)$, thickness ratio $\left(h_{S}^{*}\right)$, and the density ratio $\left(\rho^{*}\right)$. Based on a control volume analysis scaling arguments were used to identify different regimes in the parameter space. For the two sources of forces, i.e. hydrodynamic impulse term and added mass term, the following scaling was found

(i) Strouhal number is proportional to total fluid dynamic force felt on the wing: Increasing Strouhal number will increase both the hydrodynamic impulse and the added mass terms.

(ii) As the Reynolds number and the reduced frequency increase, the fluid dynamic force felt on a moving body, such as a rigid or deforming wing in air or water, will be dominated by the added mass term, which is proportional to the acceleration of the body motion.

For flexible wings flapping in water or air, the force generation and the propulsive efficiency of the wings were considered. Although the previous studies (e.g. $[70,71,25,26]$ ) showed that the fluid dynamic force on the wings is negligible compared to the inertial force, to account for the low density ratio effects, the fluid force due to added mass is additionally considered. For the cases considered, where the Reynolds number is high in the order of $O\left(10^{3}-10^{4}\right)$ and high reduced frequency $O(1)$, the followings were shown.

(iii) The tip deformation is an outcome of the interplay between the imposed kinematics and the response of the wing structure dictated by the wing tip amplitude and the phase lag. The amplitude of the maximum relative wing tip deformation, $\gamma$, was obtained from the beam analysis and is only function of the a priori known non-dimensional paramters. By considering the energy balance of the wing the time-averaged force normalized by the effective stiffness was related to $\gamma$, and a scaling was established as $\left\langle C_{F}\right\rangle=\Pi_{1} \Psi(\gamma)$ where $\left\langle C_{F}\right\rangle$ is the force that is responsible for the wing deformation.

(iv) The time-averaged force $\left\langle C_{F}\right\rangle$ can be related to the resultant force on the wing depending on the situation, such as fluid/inertial force, with/without freestream, or thrust/lift/weight. The results of current study enable us to estimate the order of magnitude of the time-averaged force generation for a flexible flapping wing using a priori known parameters.

Furthermore, for the propulsive efficiency, defined as the ratio between the time-averaged propulsive force and the power input, similar scaling analysis was performed:

(v) The power input, which is equivalent to the work done during one motion cycle, could be scaled by accounting for the wing deformations which introduces a phase lag in the resulting fluid dynamic force with respect to the motion.

(vi) The power input scales with $S t^{2}$ for low frequency ratio $\left(f / f_{1}\right)$ motions. For higher frequency ratio motions the effects of the inertia and the stiffness of the wing will influence the power input.

(vii) The propulsive efficiency scales with $S t^{2}\left\langle C_{T}\right\rangle$ at low frequency ratios for forward flight. In this regime increasing propulsion results in higher propulsive efficiency. For higher frequency ratio motions the increase of the power input will overshadow the propulsion, deterioating the efficiency.

(viii) Optimal frequency ratio for the propulsive efficiency was found to be 0.41 of the natural frequency of the wing.

(ix) The current scaling shows, consistent with Shyy et al. [3] that smaller flyers need to flap faster from the efficiency point of view, but the relative payload capacity increases because its weight reduces at a much faster rate compared to a larger flyers.

In summary, a relationship between the time-averaged force normalized by the effective stiffness and the maximum wing tip deformation relative to the imposed kinematics is established by considering the energy balance of the wing. Furthermore, the lift generation of insect flyers, approximated by its weight, largely follows the same scaling relationship. Similar scaling analysis are performed for the power input and propulsive efficiency. The chosen scaling parameters, given as a combination of a priori known wing geometry, structural properties, and motion amplitude and frequency, helps to gain more insight in the combined fluid and structural dynamics and is applicable to a wide range of scenarios involving different motion types, Reynolds numbers, and the fluid media regarding the magnitude of the time-averaged force, which can guide the design of flapping wing micro air vehicles. 


\section{Acknowledgments}

The work was sponsored in part by the Air Force Office of Scientific Research's Multidisciplinary University Research Initiative (MURI) grant and by the Michigan/AFRL (Air Force Research Laboratory)/Boeing Collaborative Center in Aeronautical Sciences.

\section{Appendix A Spatial and Temporal Sensitivity Studies}

\section{A.1 Plunging Chordwise Flexible Airfoil in Water at $\mathrm{Re}=\mathbf{9} \times 10^{3}$}

Unstructured grids around an airfoil with a rigid teardrop and a flexible flatplate are utilized to solve for the flow field. In order to identify the suitable number of grid points and the time step, grid and time step sensitivity analyses are performed for the rigid airfoil at $S t=0.17$. From the results shown in Figure A-5, the Intermediate grid $\left(25 \times 10^{3}\right.$ cells) and $T / d t=480$ are chosen. As to the prescribed wing motion, the rigid teardrop is actuated by a sinusoidal plunge displacement with the normalized amplitude of 0.194 . The fluid dynamics computation is assumed to be fully turbulent and the Menter's SST turbulence model is used. The $y^{+}$of the first grid spacing is set to be of the order of $O(1)$. For the outer boundary conditions, located at 25 chords away from the airfoil, the freestream velocity, density, and turbulence quantities are assigned. On the airfoil surface the noslip condition is imposed. In order to compute the wing deformations, a finite element Euler-Bernoulli beam model with 51 nodes is utilized.

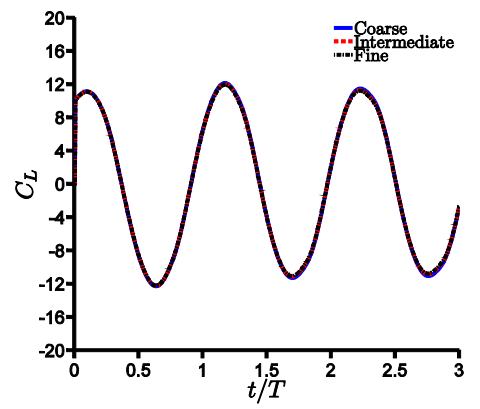

(a) lift coefficient

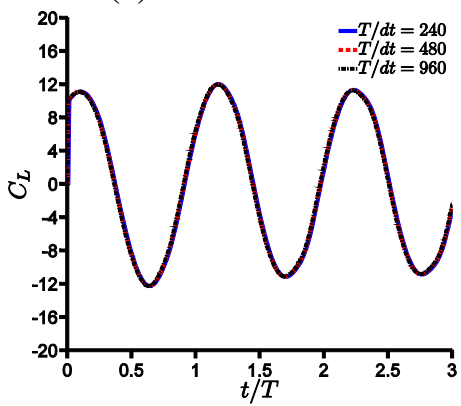

(c) lift coefficient

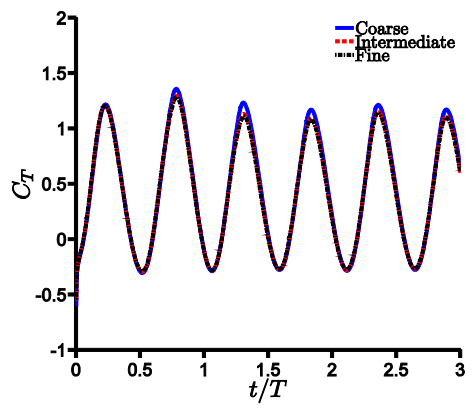

(b) thrust coefficient

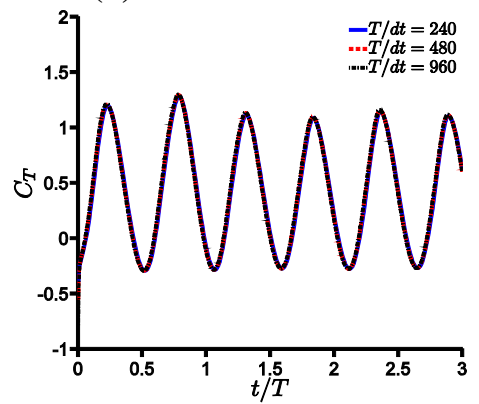

(d) thrust coefficient

Figure A-5 Temporal and spatial sensitivity analyses for a rigid plunging airfoil at $S t=0.17$. Grid sizes are Coarse: $6 \times 10^{3}$, Intermediate: $25 \times 10^{3}$, and Fine: $100 \times 10^{3}$ cells.

\section{A.2 Plunging Spanwise Flexible Wing in Water at $\mathrm{Re}=3 \times 10^{4}$}

Unstructured grids around a rectangular wing of a NACA0012 airfoil with $A R=3$ are utilized to solve for the flow field. In order to identify the suitable number of grid points and the time step, grid and time step sensitivity analyses are performed for the rigid wing. From the results shown in Figure A-6, the Intermediate grid $\left(0.31 \times 10^{6}\right.$ cells) and $T / d t=500$ are chosen. As to the prescribed wing motion, the wing root is actuated by a sinusoidal plunge 
displacement with the normalized amplitude of 0.175 . The fluid dynamics computation is assumed to be fully turbulent and is solved using the Menter SST turbulence model. The $y^{+}$of the first cell away from the wing is set to be of the order of $O(1)$. For the outer boundary conditions, located around 20 chords away in radial direction from the wing and 9 chords from the wing tip, the freestream velocity, density, and turbulence quantities are assigned. On the wing surface the noslip condition is imposed. In order to compute the wing deformations, a triangle facet shell finite-element discretization with 768 elements is utilized. The degrees of freedom of the node relevant to the chordwise displacement are constrained since Heathcote, Wang, and Gursul [65] observed that the degree of chordwise flexion of the wing for all wings and all motion frequencies was negligible. In addition, the contribution of the poludimethysiloxane (PDMS) rubber material which was used in the experimental wing configuration to the overall mass and stiffness properties is assumed to be negligible; therefore only the stainless steel for the Flexible wing with $\rho^{*}=7.8$ and $\Pi_{1}=212$ and the Very Flexible wing with $\rho^{*}=2.7$ and $\Pi_{1}=38$ is considered. Note that the material properties of the Very Flexible wing are based on a static bending test (private communications with Drs. Wang and Gursul at University of Bath).

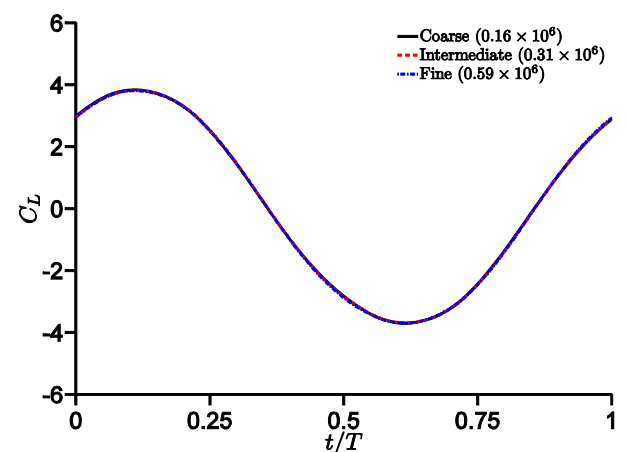

(a) lift coefficient

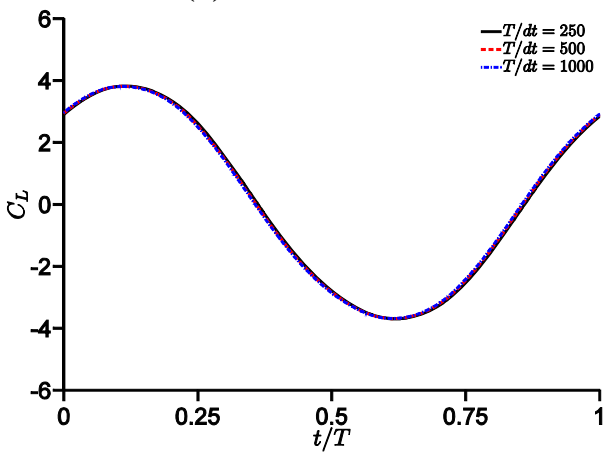

(c) lift coefficient

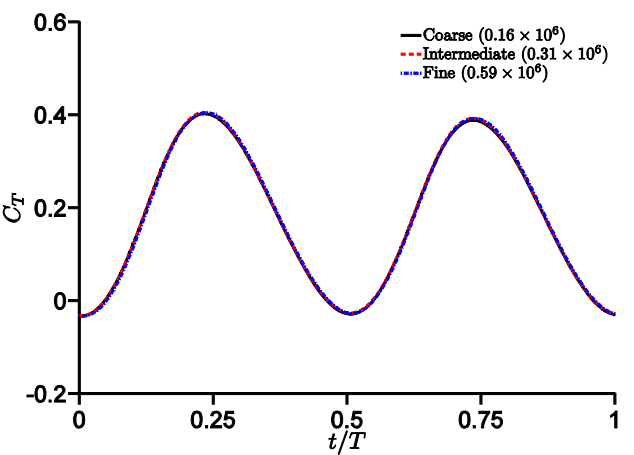

(b) thrust coefficient

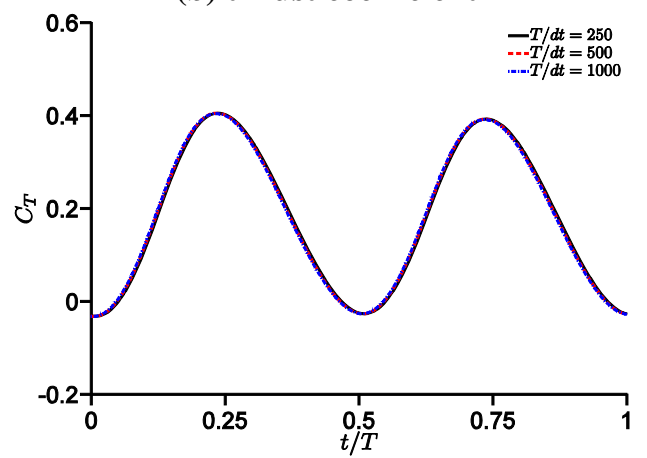

(d) thrust coefficient

Figure A-6 Temporal and spatial sensitivity analyses for a rigid plunging NACA0012 wing at $k=1.82$. Grid sizes are Coarse: $0.16 \times 10^{6}$, Intermediate: $0.31 \times 10^{6}$, and Fine: $0.59 \times 10^{6}$ cells.

\section{A.3 Flapping Zimmerman Wing in Air at $\mathrm{Re}=1.5 \times 10^{3}$}

To assess the grid size sensitivity three grids with different spatial resolutions around a Zimmerman wing are utilized for the fluid dynamic computation. The mesh to solve for the fluid equations consists of mixed brick near the wing and tetrahedral cells away from the wing. For the time step sensitivity analysis 250, 500, and 1000 time steps per motion cycle were chosen. The computational fluid dynamics (CFD) and the computational structural dynamics' (CSD) grid configurations are shown in Figure A-7. From the results shown in Figure A-8, the intermediate grid with $0.51 \times 10^{6}$ nodes and 500 time steps per motion cycle show grid and time step independent solution. The fluid flow is assumed to be laminar. The first grid spacing from the wing surface is set to $2.5 \times 10^{-3}$ and the outer boundary of the computational grid is located at 30 chords away from the wing. At the outer computational boundary zero velocity and reference density are assigned. On the wing surface the noslip condition is applied. In order to compute the wing deformations, a triangle facet shell finite-element discretization with 767 elements is used. 


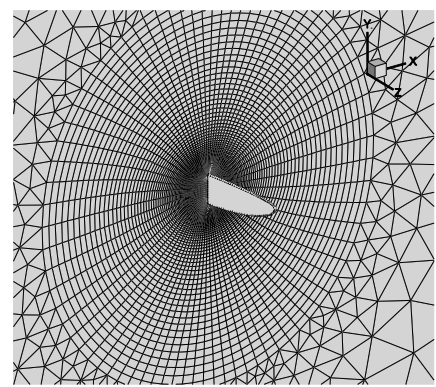

(a) CFD mesh

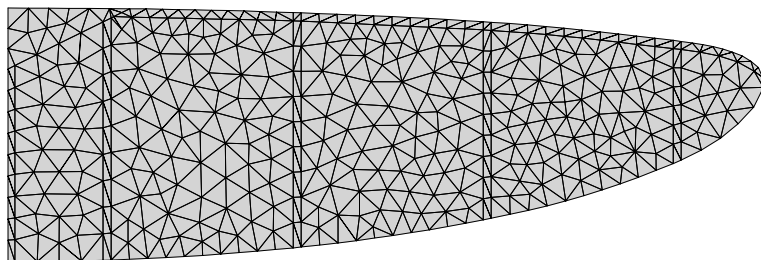

(b) CSD mesh

Figure A-7 Computational grids for the flapping isotropic Zimmerman wing in hover in air.

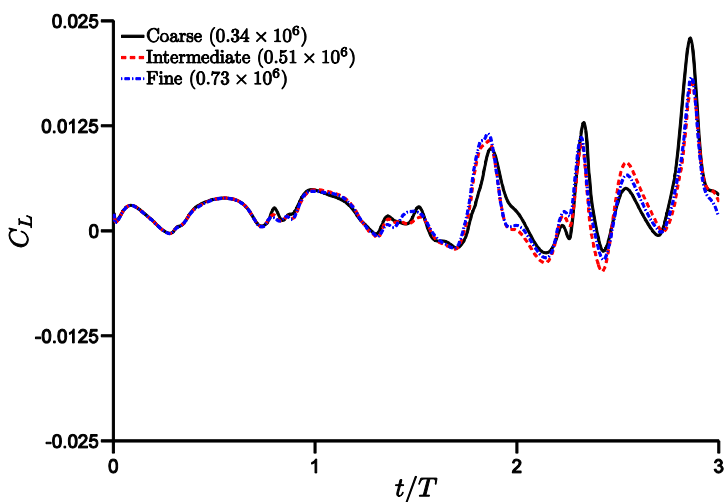

(a) lift coefficient

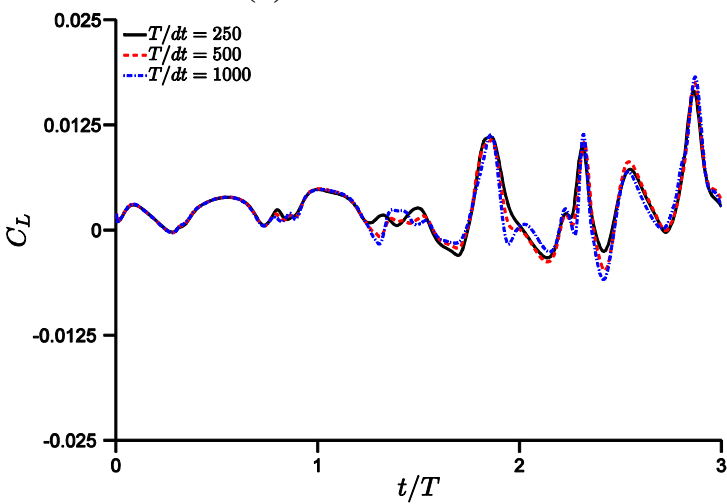

(c) lift coefficient

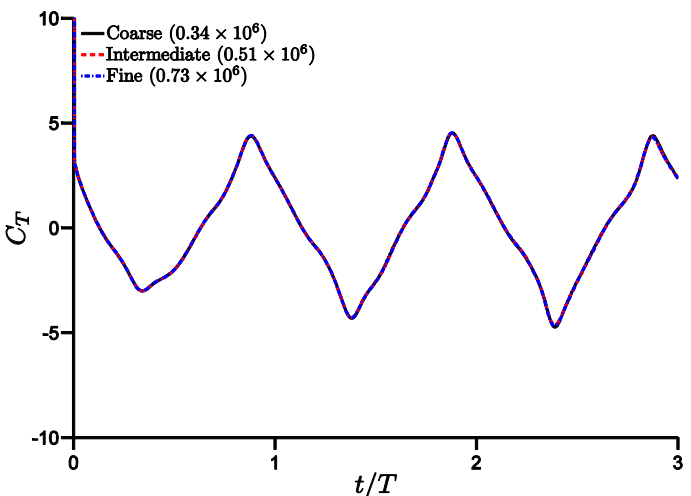

(b) thrust coefficient

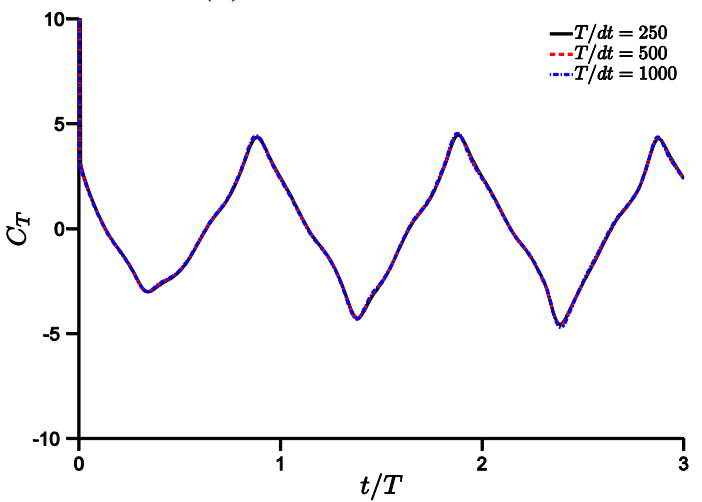

(d) thrust coefficient

Figure A-8 Temporal and spatial sensitivity analyses for a rigid flapping Zimmerman wing in hover at $R e=$ $1.5 \times 10^{3}, k=0.56$, and $S t=0.25$. Grid sizes are Coarse: $0.34 \times 10^{6}$, Intermediate: $0.51 \times 10^{6}$, and Fine: $0.73 \times 10^{6}$ cells.

\section{Appendix B Training and Testing Points in the Design of Experiment of the Hovering Zimmerman Wing Case}

Tabel B-1 shows the lift of training and testing pointes in the design space for the flapping isotropic Zimmerman wing in hover in air (Section V.1.C). The $\Pi_{1}$ and $\rho^{*}$ are the design variables. In the computations the Young's moduli and the density of the wing are varied.

Different weighting strategies are employed to minimize the risk of generating surrogates that fit the training 
data well but perform less in other regions. The weighted average surrogates (WAS) use constant weights, meaning that a certain surrogate will have the same importance throughout the design space. The Polynomial Response Surface, Kriging, and Support Vector Regression are used for the individual surrogates, after which each surrogate is weighted in correlation to the RMS PRESS values, defined as

$$
\text { RMS PRESS }=\sqrt{\frac{1}{N_{s}} \sum_{i=1}^{N_{S}}\left(y_{i}-\hat{y}_{i}^{(-i)}\right)}
$$

where $\hat{y}_{i}^{(-i)}$ is the prediction at $x^{(i)}$ using a surrogate model constructed with all training point except $x^{(i)}$, and $N_{s}$ is the number of training points. Table B-2 shows the RMS PRESS values as predicted by the individual surrogate models for $\left\langle C_{L}\right\rangle, \psi$, and $\theta$. The cells with underlines indicate the lowest RMS PRESS values and the surrogates that are weighted in the WAS.

Table B-1 List of training points $(1-14)$ and testing points $(15,16)$ in the design space for the flapping isotropic Zimmerman wing case. $\Pi_{1}$ and $\rho^{*}$ are the design variables.

\begin{tabular}{ccccc}
\hline \hline Case Nr. & $\Pi_{1}$ & $\rho^{*}$ & $E$ & $\rho_{S}$ \\
\hline 1 & $1.0 \times 10^{2}$ & $1.00 \times 10^{1}$ & $2.00 \times 10^{8}$ & $1.23 \times 10^{1}$ \\
2 & $1.04 \times 10^{5}$ & $1.00 \times 10^{1}$ & $2.00 \times 10^{11}$ & $1.23 \times 10^{1}$ \\
3 & $1.04 \times 10^{5}$ & $1.00 \times 10^{4}$ & $2.00 \times 10^{11}$ & $1.23 \times 10^{4}$ \\
4 & $1.00 \times 10^{3}$ & $1.00 \times 10^{3}$ & $1.92 \times 10^{9}$ & $1.23 \times 10^{3}$ \\
5 & $3.29 \times 10^{3}$ & $3.16 \times 10^{2}$ & $6.32 \times 10^{9}$ & $3.89 \times 10^{2}$ \\
6 & $1.04 \times 10^{5}$ & $3.16 \times 10^{2}$ & $2.00 \times 10^{11}$ & $3.89 \times 10^{2}$ \\
7 & $3.29 \times 10^{3}$ & $1.00 \times 10^{1}$ & $6.32 \times 10^{9}$ & $1.23 \times 10^{1}$ \\
8 & $4.38 \times 10^{4}$ & $2.19 \times 10^{3}$ & $8.41 \times 10^{10}$ & $2.69 \times 10^{3}$ \\
9 & $1.00 \times 10^{4}$ & $1.00 \times 10^{2}$ & $1.92 \times 10^{10}$ & $1.23 \times 10^{2}$ \\
10 & $3.16 \times 10^{2}$ & $3.16 \times 10^{1}$ & $6.07 \times 10^{8}$ & $3.89 \times 10^{1}$ \\
11 & $1.00 \times 10^{2}$ & $1.00 \times 10^{2}$ & $2.00 \times 10^{8}$ & $1.23 \times 10^{2}$ \\
12 & $1.00 \times 10^{3}$ & $1.00 \times 10^{2}$ & $1.92 \times 10^{9}$ & $1.23 \times 10^{2}$ \\
13 & $1.00 \times 10^{4}$ & $1.00 \times 10^{3}$ & $1.92 \times 10^{10}$ & $1.23 \times 10^{3}$ \\
14 & $1.00 \times 10^{4}$ & $1.00 \times 10^{4}$ & $1.92 \times 10^{10}$ & $1.23 \times 10^{4}$ \\
15 & $5.86 \times 10^{2}$ & $3.06 \times 10^{2}$ & $1.13 \times 10^{9}$ & $3.77 \times 10^{2}$ \\
16 & $3.78 \times 10^{3}$ & $5.72 \times 10^{1}$ & $7.26 \times 10^{9}$ & $7.04 \times 10^{1}$ \\
\hline \hline
\end{tabular}

Table B-2 RMS PRESS values as predicted by the individual surrogate models for the lift, twist, and bending angle. Surrogate models indicated by the underlines are used for the WAS construction.

\begin{tabular}{ccccccccc}
\hline \hline$\left[10^{-1}\right]$ & KRG & PRS & SVR1 & SVR2 & SVR3 & SVR4 & SVR5 & SVR6 \\
\hline$\left\langle C_{T}\right\rangle$ & 3.56 & 3.57 & 2.93 & 2.23 & 2.54 & 5.57 & 2.44 & 2.38 \\
$\psi$ & 3.65 & 6.65 & 2.77 & 2.47 & 2.64 & 2.67 & 1.98 & 2.35 \\
$\theta$ & 3.63 & 3.62 & 2.63 & 2.05 & 2.51 & 2.53 & 1.32 & 2.47 \\
\hline \hline
\end{tabular}

Finally, the error measured at the independent testing points defined as the relative difference between the constructed WAS and the actual values from the simulations with respect to the range of the objective functions show, see Table B-3, that for the case 15 where the high gradients in the surrogates are located has larger error than for the more stiffer case 16 . 
Table B-3 Relative error at the independent testing points 15 and 16. The difference in the value predicted by the surrogate model and the numerical computations are normalized by the range of the surrogate response

\begin{tabular}{ccc}
\hline \hline Objective function & Relative error at testing point 15 (\%) & Relative error at testing point 16 (\%) \\
\hline$\left\langle C_{T}\right\rangle$ & 23 & 8 \\
$\theta$ & 15 & 5 \\
$\psi$ & 7 & 5 \\
\hline \hline
\end{tabular}

\section{Appendix C Modal analysis of isotropic Zimmerman wing}

Natural frequencies are computed using MSC.Marc and shown in Table C-1. Moreover, the mode shapes of the wing for each natural frequency are illustrated in Figure C-1. The first mode is related to the spanwise bending of the wing and the second mode the twist of the wing. Note that all the natural frequencies listed in Table C-1 are higher than the exciatation frequency of $10 \mathrm{~Hz}$.

Table C-1 First two natural frequencies in $\mathrm{Hz}$ based on a modal analysis for the training and testing points in the design space for flapping isotropic Zimmerman wing case.

\begin{tabular}{ccc}
\hline \hline Case Nr. & Mode 1 & Mode 2 \\
\hline 1 & $7.46 \times 10^{1}$ & $3.04 \times 10^{2}$ \\
2 & $2.36 \times 10^{3}$ & $9.61 \times 10^{3}$ \\
3 & $7.46 \times 10^{1}$ & $3.04 \times 10^{2}$ \\
4 & $2.31 \times 10^{1}$ & $9.41 \times 10^{1}$ \\
5 & $7.46 \times 10^{1}$ & $3.04 \times 10^{2}$ \\
6 & $4.19 \times 10^{2}$ & $1.71 \times 10^{3}$ \\
7 & $4.19 \times 10^{2}$ & $1.71 \times 10^{3}$ \\
8 & $1.03 \times 10^{2}$ & $4.21 \times 10^{2}$ \\
9 & $2.31 \times 10^{2}$ & $9.41 \times 10^{2}$ \\
10 & $7.31 \times 10^{1}$ & $2.98 \times 10^{2}$ \\
11 & $2.36 \times 10^{1}$ & $9.61 \times 10^{1}$ \\
12 & $7.31 \times 10^{1}$ & $2.98 \times 10^{2}$ \\
13 & $7.31 \times 10^{1}$ & $2.98 \times 10^{2}$ \\
14 & $2.31 \times 10^{2}$ & $9.41 \times 10^{2}$ \\
15 & $3.20 \times 10^{1}$ & $1.31 \times 10^{2}$ \\
16 & $1.88 \times 10^{2}$ & $7.65 \times 10^{2}$ \\
\hline \hline
\end{tabular}

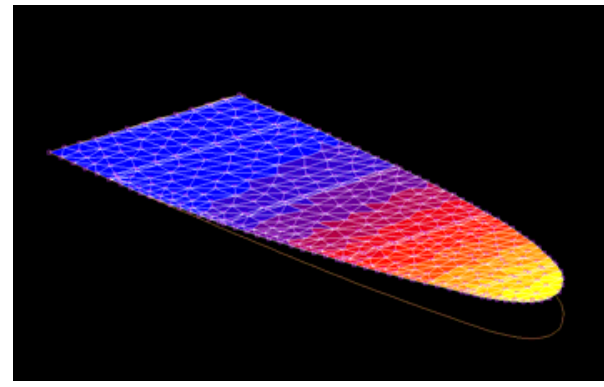

Mode 1

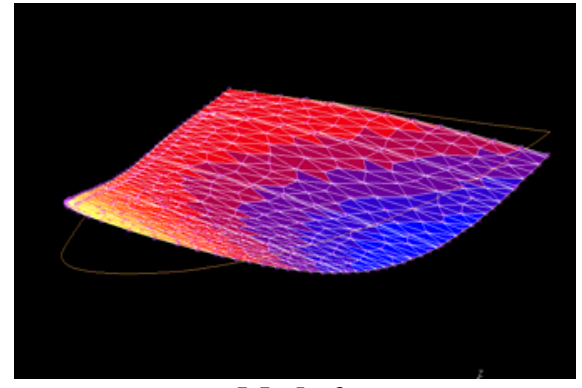

Mode 2

Figure C-1. Snapshots of the wing shapes for the first four modes based on a modal analysis for the training and testing points in the design space for the flapping isotropic Zimmerman wing case. 


\section{References}

[1] T Reichert, C Robertson, and J. D. DeLaurier. (2010, Sep.) Human-powered Ornithopter Becomes First Ever to Achieve Sustained Flight. [Online]. http://www.engineering.utoronto.ca/About/Engineering_in_the_News/Humanpowered_Ornithopter_Becomes_First_Ever to Achieve_Sustained Flight.htm

[2] T.J. Muller, Fixed and Flapping Wing Aerodynamics for Micro Air Vehicle Applications. New York: AIAA, 2002.

[3] W. Shyy, Y. Lian, J. Tang, D. Viieru, and H. Liu, Aerodynamics of Low Reynolds Number Flyers.: Cambridge Univ Press, 2008.

[4] W. Shyy, M. Berg, and D. Ljungqvist, "Flapping and Flexible Wings for Biological and Micro air Vehicles," Progress in Aerospace Sciences, vol. 24, no. 5, pp. 455 - 505, 1999.

[5] D. Floreano, J.-C. Zufferey, M. Srinivasan, and C. Ellington, Flying Insects and Robots. Berlin, Germany: Springer-Verlag, 2009.

[6] W. Shyy, H. Aono, S. K. Chimakurthi, P. Trizila, C.-K. Kang, C. E. S. Cesnik, and H. Liu, "Recent progress in flapping wing aerodynamics and aeroelasticity," Progress in Aerospace Sciences, vol. 46, no. 7, pp. 284-327, 2010.

[7] Aerovironment. (2011, Feb.) Aerovironment Inc. [Online]. http://www.avinc.com/nano

[8] Festo. (2011, Mar.) Festo. [Online]. http://www.festo.com/cms/en_corp/11369.htm

[9] K. M. E. de Clercq, R. de Kat, B. Remes, B. W. van Oudheusden, and H. Bijl, "Flow Visualization and Force Measurements on a Hovering Flapping-Wing MAV," in 39th AIAA Fluid Dynamics Conference, San Antonio, Texas, 22-25 June 2009, AIAA-2009-4035.

[10] R. J. Wood, "The First Takeoff of a Biologically Inspired At-Scale Robotic Insect," IEEE Transactions on Robotic, vol. 24, no. 2, pp. 341 - 347, 2008.

[11] A. Azuma, The Biokinetics of Flying and Swimming, 2nd ed. Virginia: AIAA, Inc., 2006.

[12] U. M. Norberg, Vertebrate Flight: Mechanics, Physiology, Morphology, Ecology, and Evolution. New York: Springer, 1990.

[13] R. Dudley, The Biomechanics of Insect Flight: Form, Function, Evolution. New Jersey: Princeton University Press, 2002.

[14] A.A. Biewener, Animal Locomotion. New York: Oxford University Press, 2003.

[15] T. Weis-Fogh, "Quick Estimates of Flight Fitness in Hovering Animals, including Novel Mechanism for Lift Production," Journal of Experimental Biology, vol. 59, pp. 169 - 230, 1973.

[16] T. Maxworthy, "The fluid dynamics of insect flight," Annual Review of Fluid Mechanics, vol. 13, pp. 329 350, 1981.

[17] S.P Sane, "The Aerodynamics of Insect Flight," Journal of Experimental Biology, vol. 206, pp. 4191 - 4208 , 2003.

[18] B.W. Tobalske, "Biomechanics of Bird Flight," Journal of Experimental Biology, vol. 210, pp. 3135 - 3146 , 2007.

[19] G. I. Barenblatt, Scaling.: Cambridge University Press, 2003.

[20] G. I. Barenblatt, Scaling, Self-similarity, and Intermediate Asymptotics.: Cambridge University Press, 1996.

[21] W.E. Baker, P.S. Westine, and F.T. Dodge, Similarity Methods in Engineering Dynamics.: Elsevier, 1991.

[22] R. L. Bisplinghoff, H. Ashley, and R. L. Halfman, Aeroelasticity.: Dover publications, inc., 1996.

[23] D. Ishihara, T. Horie, and M. Denda, "A two-dimensional computational study on the fluid-structure interaction cause of wing pitch changes in dipteran flapping flight," Journal of Experimental Biology, vol. 212, no. 1, pp. 1-10, 2009.

[24] D. Ishihara, Y. Yamashita, T. Horie, S. Yoshida, and T. Niho, "Passive maintenace of high angle of attack and its generation during flapping translation in crane fly wing," Journal of Experimental Biology, vol. 212, pp. 3882-3891, 2009.

[25] B. Thiria and R. Godoy-Diana, "How wing compliance drives the efficiency of self-propelled flapping flyers," Physical Review E, vol. 82, no. 1, p. 015303, 2010. 
[26] S. Ramananarivo, R. Godoy-Diana, and B. Thiria, "Rather than resonance, flapping wing flyers may play on aerodynamics to improve performance," Proceedings of the National Academy of Sciences, vol. 108, no. 15, pp. 5964-5969, 2011.

[27] J. Zhang, N.-S. Liu, and X.-Y. Lu, "Locomotion of a passively flapping flat plate," Journal of Fluid Mechanics, vol. 659 , pp. 43 - $68,2010$.

[28] H. Masoud and A. Alexeev, "Resonance of flexible flapping wings at low Reynolds number," Physical Review E, vol. 81, p. 056304, 2010.

[29] A. Gogulapati and P. Friedmann, "Approximate Aerodynamic and Aeroelastic Modeling of Flapping Wings in Hover and Forward Flight," in 52nd AIAA/ASME/ASCE/AHS/ASC Structures, Structural Dynamics and Materials Conference 19th AIAA/ASME/AHS Adaptive Structures Conference, Denver, CO, 4 - 7 April, 2011, AIAA-2011-2008.

[30] S. Michelin and S.G. Llewellyn Smith, "Resonance and Propulsion Performance of a Heaving Flexible Wing," Physics of Fluids, vol. 21, p. 071902, 2009.

[31] P. Wu, P. Ijfu, and B. Stanford, "Flapping wing structural deformation and thrust correlation study with flexible membrane wings," AIAA Journal, vol. 48, no. 9, pp. 2111-2122, 2010.

[32] S. Sunada, L. Zeng, and K. Kawachi, "The relationship between dragonfly wing structure and torsional deformation," Journal of Theoretical Biology, vol. 193, pp. 39 - 45, 1998.

[33] J.-S. Chen, J.-Y. Chen, and Y.-F. Chou, "On the natural frequencies and mode shapes of dragonfly wings," Journal of Sound and Vibration, vol. 313, pp. 643-654, 2008.

[34] M. Vanella, T. Fitzgerald, S. Preidikman, E. Balaras, and B. Balachandran, "Influence of flexibility on the aerodynamic performance of a hovering wing," Journal of Experimental Biology, vol. 212, pp. 95-105, 2009.

[35] Wei Shyy. (2010) Flight InSight. [Online]. http://shyylab.engin.umich.edu/flightinsight

[36] M.S. Triantafyllou, G.S. Triantafyllou, and D.K.P. Yue, "Hydrodynamics of fishlike swimming," Annual Review of Fluid Mechanics, vol. 32, pp. 33 - 53, 2000.

[37] J. A. Wright and R. W Smith, "An edge-based method for the incompressible Navier-Stokes equations on polygonal meshes," Journal of Computational Physics, vol. 169, pp. 24-43, 2001.

[38] R. W. Smith and J. A. Wright, "An implicit edge-based ALE method for the incompressible Navier-Stokes equations," International Journal for Numerical Methods in Fluids, vol. 43, pp. 253-279, 2003.

[39] R. Kamakoti, S. Thakur, J. Wright, and W. Shyy, "Validation of a new parallel all-speed cfd code in a rulebased framework for multidisciplinary applications," in 36th AIAA Fluid Dynamics Conference and Exhibit, San Francisco, California, June 5-8, 2006, AIAA-2006-3063.

[40] S. Balay, W. D. Gropp, L. C. McInnes, and B. F. Smith, "Efficient management of parallelism in object oriented numerical software libraries," in Modern Software Tools in Scientific Computing, 1997, pp. 163-202.

[41] S. Balay, J. Brown, K. Buschelman, V. Eijkhout, W. D. Gropp, D. Kaushik, M. G. Knepley, L. C. McInnes, B. F. Smith, and H. Zhang, "PETSc Users Manual," Argonne National Laboratory, 2010.

[42] S. Balay, J. Brown, K. Buschelman, W. D. Gropp, D. Kaushik, M. G. Knepley, L. C. McInnes, B. F. Smith, and H. Zhang. (2011) PETSc Web page. [Online]. http://www.mcs.anl.gov/petsc

[43] R. Falgout and U. Yang, "hypre: A library of high performance preconditioners," in Computational Science ICCS 2002, Peter Sloot, Alfons Hoekstra, C. Tan, and Jack Dongarra, Eds.: Springer Berlin / Heidelberg, 2002, pp. 632-641.

[44] E. A. Luke and T. George, "Loci: a rule-based framework for parallel multi-disciplinary simulation synthesis," Journal of Functional Programming, vol. 15, no. 03, pp. 477-502, 2005.

[45] P.D. Thomas and C. K. Lombard, "Geometric conservation law and its application to flow computations on moving grids," AIAA Journal, vol. 17, no. 10, pp. 1030-1037, 1979.

[46] R. Kamakoti and W. Shyy, "Evaluation of geometric conservation law using pressure-based fluid solver and moving grid technique," International Journal of Heat and Fluid Flow, vol. 14, no. 7, pp. 851-865, 2004.

[47] A. de Boer, M. S. van, and H. Bijl, "Mesh deformation based on radial basis function interpolation," Computers \& Structures, vol. 85, no. 11-14, pp. 784-795, 2007.

[48] F.R. Menter, M. Kuntz, and R. Langtry, "Ten Years of Industrial Experience with the SST Turbulence Model. In: Hanjalic, K., Nagano, Y., Tummers, M. (Eds.)," Turbulence, Heat and Mass Transfer 4, Begell House, pp. 
$625-632,2003$.

[49] C. Kang, H. Aono, P. Trizila, Y. Baik, J. M. Rausch, L. Bernal, M. V. Ol, and W. Shyy, "Modeling of Pitching and Plunging Airfoils at Reynolds Number between 1x10^4 and 6x10^4," in 27th AIAA Applied Aerodynamics Conference, San Antonio, Texas, June 22-25,2009, AIAA-2009-4100.

[50] P. Khosravi, R. Ganesan, and R. Sedaghati, "Corotational non-linear analysis of thin plates and shells using a new shell element," International Journal for Numerical Methods in Engineering, vol. 69, no. 4, pp. 859-885, 2007.

[51] S. K. Chimakurthi, C. E. S. Cesnik, and B. Stanford, "Flapping-wing structural dynamics formulation based on a corotational shell finite element," AIAA Journal, vol. 49, no. 1, pp. 128-142, 2011.

[52] S. K. Chimakurthi, J. Tang, R. Palacios, C. E. S., and W. Shyy, "Computational aeroelasticity framework for analyzing flapping wing micro air vehicles," AIAA Journal, vol. 47, pp. 1865-1878, 2009.

[53] U. Küttler and W. A. Wall, "Fixed-point fluid-structure interaction solvers with dynamic relaxation," Computational Mechanics, vol. 43, pp. 61-72, 2008, 10.1007/s00466-008-0255-5.

[54] C. Kang, H. Aono, C.E.S. Cesnik, and W. Shyy, "A Scaling Parameter for Thrust Generation of Flapping Flexible Wings," in 49th AIAA Aerospace Sciences Meeting, Orlando, Florida, 4 - 7 January 2011, AIAA-20111313.

[55] F. Noca, "On the evaluation of time-dependent fluid-dynamic forces on bluff bodies," California Institute of Technology, $\mathrm{PhD}$ thesis 1997.

[56] P. G. Saffman, Vortex dynamics.: Cambridge Univ Press, 1995.

[57] M. R. Visbal, Gordnier R. E., and M. C. Galbraith, "High-fidelity Simulations of Moving and Flexible Airfoils at Low Reynolds Numbers," Experiments in Fluids, vol. 46, pp. 903 - 922, 2009.

[58] P. Trizila, C.-K. Kang, H. Aono, M. Visbal, and W. Shyy, "Low-Reynolds-number aerodynamics of a flapping rigid flat plate," AIAA Journal, vol. 49, no. 4, pp. 806-823, 2011.

[59] W. Shyy, P. Trizila, C.-K. Kang, and H. Aono, "Can Tip Vortices Enhance Lift of a Flapping Wing?," AIAA Journal, vol. 47, pp. 289 - 293, 2009.

[60] William Graebel, Advanced fluid mechanics.: Academic Press, 2007.

[61] J. Katz and A. Plotkin, Low-speed aerodynamics.: Cambridge University Press, 2001.

[62] T. Theodorsen, "General theory of aerodynamic instability and the mechanism of flutter," NACA report 496, 1935.

[63] M. Ol, L. Bernal, C.-K. Kang, and W. Shyy, "Shallow and deep dynamic stall for flapping low Reynolds number airfoils," Experiments in Fluids, vol. 46, no. 5, pp. 883-901, 2009-05-01.

[64] S. Heathcote and I. Gursul, "Flexible flapping airfoil propulsion at low Reynolds numbers," AIAA Journal, vol. 45, no. 5, pp. 1066-1079, 2007.

[65] S. Heathcote, Z. Wang, and I. Gursul, "Effect of spanwise flexibility on flapping wing propulsion," Journal of Fluids and Structures, vol. 24, no. 2, pp. 183-199, 2008.

[66] R. E. Gordnier, P. J. Attar, S. K. Chimakurthi, and C. E. S. Cesnik, "Implicit LES simulations of a flexible flapping wing," in 18th AIAA/ASME/AHS Adaptive Structures Conference, Orlando, FL, 12 - 15 April, 2010, AIAA-2010-2960.

[67] H. Aono, S. K. Chimakurthi, P. Wu, E. Sällström, B. K. Stanford, C. E. S., P. Ifju, L. Ukeiley, and W. Shyy, "A computational and experimental study of flexible flapping wing aerodynamics," in 48th AIAA Aerospace Sciences Meeting Including the New Horizons Forum and Aerospace Exposition, Orlando, Florida, January 4-7, 2010, AIAA-2010-554.

[68] S. Timoshenko, D. H. Young, and JR. W. Weaver, Vibration problems in engineering.: John Wiley $\backslash \&$ Sons, Ltd., 1974.

[69] R. D. Mindlin and L. E. Goodman, "Beam vibrations with time-dependent boundary conditions," Journal of Applied Mechanics, vol. 17, pp. 353-356, 1950.

[70] T. L. Daniel and S. A. Combes, "Flexible wings and fins: bending by inertial or fluid-dynamic forces?," Integrative and Comparative Biology, vol. 42, no. 5, pp. 1044-1049, 2002.

[71] S. A. Combes and T. L. Daniel, "Into thin air: contributions of aerodynamic and inertial-elastic forces to wing bending in the hawkmoth Manduca sexta," Journal of Experimental Biology, vol. 206, no. 17, pp. 2999-3006, 
2003.

[72] J. P. Whitney and R. J. Wood, "Aeromechanics of passive rottion in flapping flight," Journal of Fluid Mechanics, vol. 660, pp. 197-220, 2010.

[73] A. P. Willmott and C. P. Ellington, "The mechanics of flight in the hawkmoth Manduca sexta. I. Kinematics of hovering and forward flight," Journal of Experimental Biology, vol. 200, pp. 2705-2722, 1997.

[74] A. P. Willmott and C. P. Ellington, "The mechanics of flight in the hawkmoth Manduca sexta. II. Aerodynamic consequences of kinematic and morphological variation," Journal of Experimental Biology, vol. 200, pp. 27232745, 1997.

[75] R. Buchwald and R. Dudley, "Limits to vertical force and power production in bumblebess (Hymenoptera: Bombus impatiens)," Journal of Experimental Biology, vol. 213, pp. 426-432, 2010.

[76] S. Vogel, "Flight in Drosophila: I. Flight performance of tethered flies," Journal of Experimental Biology, vol. 44, pp. 567-578, 1966.

[77] E. Shevtsova, C. Hansson, D. H. Janzen, and J. Kjaerandsen, "Stable structural color patterns displayed on transparent insect wings," Proceedings of the National Academy of Sciences, vol. 108, no. 213, pp. 668-673, 2011.

[78] S. A. Combes and T. L. Daniel, "Flexural stiffness in insect wings I. Scaling and the influence of wing venation," Journal of Experimental Biology, vol. 206, no. 17, pp. 2979-2987, 2003.

[79] C. J. Pennycuick, "Wingbeat Frequency of Birds in Steady Cruising Flight: New Data and Improved Predictions," Journal of Experimental Biology, vol. 199, pp. 1613 - 1618, 1996.

[80] B. Yin and H. Luo, "Effect of wing inertia on hovering performance of flexible flapping wings," Physics of Fluids, vol. 22, p. 111902, 2010.

[81] Z.J. Wang, "Aerodynamic efficiency of flapping flight: analysis of a two-stroke model," Journal of Experimental Biology, vol. 211, pp. 234 - 238, 2008. 